4888

A 5

1999

NMAA 



\title{
Department of Fine Arts
}

\section{Alaska-Yukon-Pacific Exposition}

\author{
Seattle, Washington
}

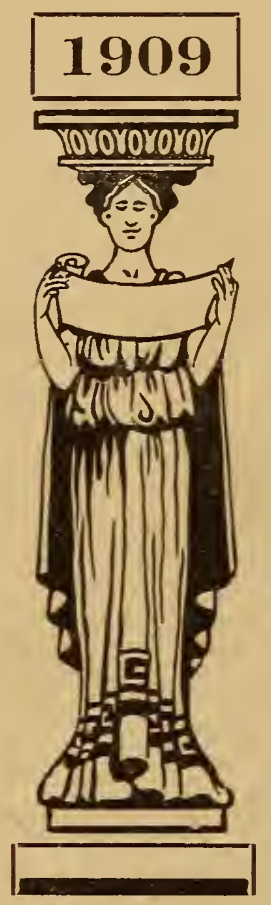

G. L. BERG, Director

CONMTTEE OF THE FINE IRTS:

DR. ALFRED RAYMONI, CHAIRMAN

J. W. CLISE, FRID. E. SANDER, H. W. TREAT, JOSEPH BLETHEN, ED. S. CURTIS.

\section{A.-Y.-P. PUBLISHING CO.}




\section{N \\ 15 \\ C.2 \\ 1904 \\ NMAA}

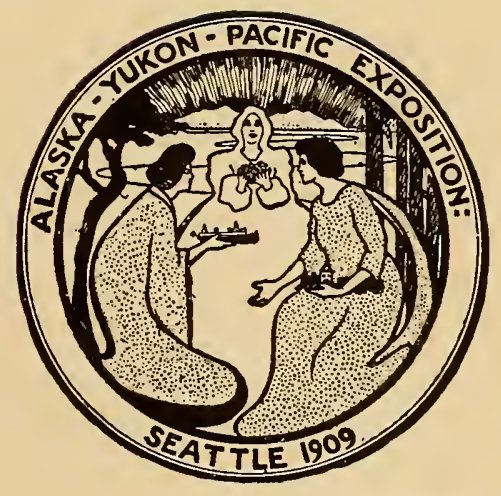

NMAA/NPG LIBRARY

\section{MAY 301990}




\section{INTRODUCTION}

Visitors to the Galleries, appreciative of the highest art, will find the best products of the artists represented.

Galleries A and B contain the most notable collection of Old Masters, early English and Barbizon men shown in any public exhibition.

The remaining Galleries, containing the works of early Americans and the foremost contemporary painters, are hung. as a comparative exhibition, showing sharp contrasts, rather than any attempt at grouping.

The high quality of the exhibit was made possible by the interest and generosity of the Artists, Galleries, Art Museums and owners of private collections. 


\section{Gallery A}

For information regarding art for sale apply at office.

LELY, SIR PETER-Born at Soest in Westphalia, 1618; died at London, 1680. Dutch by family. Pupil of Pieter de Grebber in Harlem, but on coming to England he so closely studied the manner of Van Dyck, who had just died, that his earlier portraits often approach the great master's style. Nominated Court Painter and Knighted by Charles II.

\section{Duchess of Portland.}

Loaned by T. B. Walker, Minneapolis, Minn.

COROT, JEAN-BAPTISTE-CAMILLE-Borne and died in Paris, 1796-1875. Pupil of Michallon and Bertin. Studied alone in Italy several years. One of the leaders of the Barbizon School. Medals of Salon, $1833,1848,1855$; E. U., 1867 . Diploma to Memory of Deceased Artists, 1878. Chev. Legion d'Honneur, 1846; Officer, 1867.

2. Ville d'Avray.

Loaned by S. M. Vose, Providence, R. I.

DIAZ DE LA PENA, NARCISO-VIRGILIO-French; 1809-1876. Pupil of Sigalon. Salon Medals, 1844, 1846, 1848. Chev. Legion d' Honneur, 1851. Diploma to Memory of Deceased Artists, 1878.

3. Wood Interior.

Loaned by Francis Lathrop, New York.

COROT, J. B. C.-(See 2.)

4. Evening Landscape.

Loaned by Francis Lathrop, New York.

OPIE, JOHN-English; 1761-1807.

5. Mother and Child.

Loaned by The Blakeslee Galleries, New York. 
MILLET, JEAN FRANCOIS-French; 1814-1875. Pupil of Delaroche. Salon Medals, 1853, 1864; E. U., 1867. Chev. Legion d'Honneur, 1868.

\section{Mercure enlevant les boeufs d'Argus.}

Loaned by H. T. Chapman, Brooklyn N. Y.

JACQUE, CHARLES EMILE-French; 1813-1894. Animal and Landscape Painter, Engraver and Etcher. Medals, Third Class, $1861,1863,1863$. Legion of Honor, 1867.

7. Landscape and Sheep.

Loaned by Geo. A. Dowden, Newark, N. J.

LELY, SIR PETER-(See 1.)

8. Lady Denham.

Loaned by R. C. \& N. M. Vose, Boston.

ZIEM, FELIX-Born at Beaune, France, 1821. Pupil of Art School of Dijon. His views of Venice have won him world-wide fame. Medals: Third Class, 1853, 1855; First Class, 1852. Legion d'Honneur, 1857; Officer, 1878.

9. Evening Glow in Venice.

Loaned by Geo. A. Dowden, Newark, N. J.

DUPRE, JULES-French; 1812-1889. Medal of Salon, 1833; E. U., 1867. Chev. Legion d'Honneur, 1849; Officier, 1870. One of the leaders of the Barbizon School.

10. Level Landscape.

Loaned by Francis Lathrop, New York.

MIGNARD, PIERRE-Born at Troyes, 1610; died, 1695. Portrait and allegorical painter. Pupil of Boucher and Vouet. His works are distinguished by a capitvating amiability in his forms, and a harmonious effect in coloring.

11. Portrait of Lady.

Loaned by J. B. Haggin, New York.

CUYP JACOB GERRITS (The elder)-Dortrecht; 1575-1649. An artist of considerable talent both in portraiture and landscape. Was one of the founders of the Academy of Painting at Dort.

12. Portrait of Himself.

Loaned by Chas. L. Hutchinson, Chicago.

COROT, J. B. C.-(See 2.)

13. The Cottage.

Loaned by H. T. Chapman, Brooklyn N. Y.

14. Just Before Sunrise. (See 2.)

Loaned by the Art Institute of Chicago. 
BRETON, JULES-Born at Courrieres, France, 1827; died July 5, 1906. Pupil of Drolling and Devigne. Salon Medals, 1857, 1859, 1861 (Rappel), 1872 (d'honneur. E. U. Medals, 1855, 1867 (gold); Hors Concours, 1900. Chev. Legion d'honneur, 1861; Officer, 1867; Commander, 1889; Member de l'Institut, 1886.

\section{By the Sea.}

Loaned by the Art Institute of Chicago.

ROUSSEAU, PIERRE ETIENNE THEODORE-French; 18121867. Pupil of Remond and Lethiere. One of the founders of the Barbizan School. Salon Medals, 1834, 1849. E. UU Medals, 1855, 1867, (Gr. Med. d'Honneur.) Chev. Legion d'Honneur. Diploma to Memory of Deceased Artists, 1878.

16. Sunset.

Loaned by H. T. Chapman, Brooklyn, N. Y.

ALLORI, CRISTOFORO (Called II Bronzino)-Born at Florence, Italy, 1577; died there, 1621. Pupil of his father, afterwards of Santo di Tito, and later adopted the new Florentine eclectic manner based on that of Correggio. He excelled in portrait painting.

17. Jealousy.

Loaned by The Blakeslee Galleries, New York.

TROYON, CONSTANT-French; 1810-1865. Landscape and animal painter. Pupil of Riocreux and Poupart. Visited Holland in 1847 and after 1848 began to introduce cattle into his landscapes. Medals, 1838, 1840, 1846, 1848, 1855. Chevalier Legion d'Honneur, 1849.

\section{White Cow.}

Loaned by J. B. Haggin, New York.

MORLAND, GEORGE-English; 1763-1804. The greatest painter of animals and farm scenes of the $18 \mathrm{th}$ century. Studied several years at the Royal Academy; first exhibited there in 1779 .

19. Resting by the Roadside.

Loaned by H. T. Chapman, Brooklyn, N. Y.

COROT, J. B. C.-(See 2.)

20. Giant Willows.

Loaned by H. T. Chapman, Brooklyn, N. I.

VAN MARCKE, EMILE-French landscape and animal painter. Born, 1827; died, 1891. Pupil of Troyon. Medals of Salon, 1867, 1869, 1870; E. U., 1878. Chevalier Legion d'Honneur, 1872.

\section{A Golden Autumn Day.}

Loaned by the Art Institute of Chicago.

CONSTABLE, JOHN-English, 1776-1837. Studied in Royal Academy, London. Three of his paintings exhibited in the Paris Salon in 1824 received marked attention from the French artists. The modern French School of Landscape Painting was materially influenced by Constable's work.

\section{Landscape with Cloudy Sky.}

Loaned by Francis Lathrop, New York. 
GRECO, EL (Theotokopoulos)-Born in Crete about 1547. Pupil of Titian in Venice. Painted in that city until 1570; then in Rome under the patronage of Cardinal Alexander Farnese until the latter part of 1576, when he went to Spain, settling at once in Toledo, where he died in 1614 .

23. St. Magdalene.

Loaned by The Blakeslee Galleries, New York.

JORDAENS, JACOB-Born at Antwerp, 1593; died there, 1678. Pupil of Adam Van Noort, as was Rubens, he became the follower and half Assistant of the latter.

\section{Christ Before the Doctors of Divinity.}

Loaned by Mark Arnheim, New York.

DELACROIX, FERDINAND-VICTOR-EUGENE-French, 1798 1863. Pupil of Guerin. Salon Medals, 1824, 1848. Medal of Honor, E. U., 1855. Chev. Legion d'Honneur, 1831, Officier, 1846. Commander, 1855. Member de l'Institut, 1857. "Chief of the Romantic School."

25. Tiger at Rest.

Loaned by H. T. Chapman, Brooklyn, N. Y.

SCHREYER, ADOLF-German; 1828-1899. Pupil of Stadel Institute. Made special study of the horse, anatomically and in action. Traveled much. Medals in Paris, 1864, 1865, 1867; Brussels, 1863; Vienna, 1873; Munich, 1876.

26. Arabian Horses.

Loaned by J. B. Haggin, New York.

COROT, J. B. C.-(See 2.)

27. River Landscape.

Loaned by Francis Lathrop, New York.

RIBERA, JOSEF De-Lo SPAGNOLETTO-Born at Xativa, 1558; died in Naples, 1656. Studied in Spain and Italy. Worked chiefly in Naples, where he founded a school.

28. St. Sebastian.

Loaned b: T. B. Walker, Minneapolis, Minn.

CANALETTO (Antonio Canal)-Born at Venice in 1697; died 1768. After some time spent in Rome in drawing and copying antiquities, he returned to Venice, occupying himself exclusively in producing views of that city. He visited London in 1746, at which time he painted many of his most striking views.

29. Venice.

Loaned by T. B. Walker, Minneapolis, Minn.

DIAZ, NARCISSE VIRGILE-(See 3.)

30. Forest of Fontainebleau.

Toaned by Francis Lathrop, New York. 


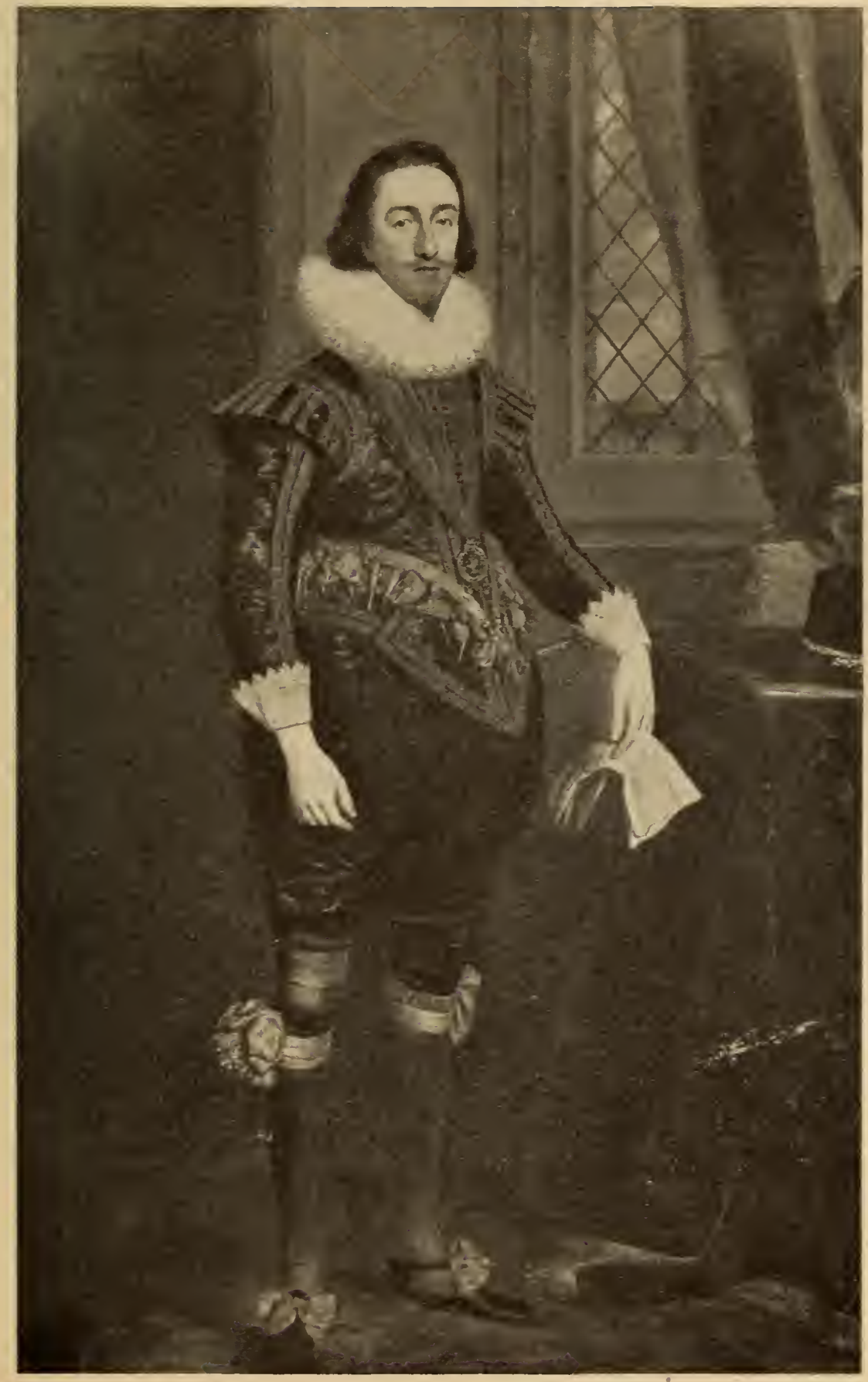

CHARLES I.

Daniel Mytens. 

DOBSON, WILLIAM-Born at Holborn in 1610; died in London October 28, 1646. After Vandyck's death, Dobson became Sergeant-Painter and groom of the Privy Chamber. He painted many notable personages.

\section{Crowning of the Virgin.}

Loaned by T. B. Walker, Minneapolis, Minn.

ROUSSEAU, THEODORE-(See 16.)

32. The Farm.

Loaned by H. T. Chapman, Brooklyn, N. Y.

CONSTABLE, JOHN-(See 22.)

33. Lock on River Stour.

Loaned by T. B. Walker, Minneapolis, Minn.

BONIFAZIO (Veronese)-Born at Verona in 1491; died, 1540. Venetian School; Pupil of Palma Vecchio, but closely imitated Titian. Forms clear and rounded, lights and shadows distinct; for color one of the first Venetian masters.

\section{Madonna and Child.}

Loaned by T. B. Walker, Minneapolis, Minn.

DUPRE, JULES-(See 10.)

35. The Old Oak.

Loaned by The Schaus Galleries, New York.

VAN RAVENSTEYN, JAN-Born at The Hague, 1572; died, 1657. Dutch school, portrait painter; painted assemblies of magistrates and corporations with great skill.

36. Dutch Lady.

Loaned by T. B. Walker, Minneapolis, Minn.

COROT, J. B. C.-(See 2.)

\section{Hillside Landscape.}

Loaned by Francis Lathrop, New York.

BOTTICELLI, SANDRO-Born at Florence, Italy, 1444. Died, 1510. First studied under Fra Fillippo Lippi. His most distinctive qualities lie in his unique power of conveying the sense of light and movement. His pictures are generally distinguished by a quaint grace of form, combined with a profound melancholy sentiment.

38. Madonna and Child and Angels.

Loaned by T. B. Walker, Minneapolis, Minn. 
VERNET, CLAUDE JOSEPH-French; 1712-1789. Marine painter. Studied in France and Rome. He became the first marine painter in Europe, and was patronized by many courts.

\section{Italian Sea Port.}

Loaned by T. B. Walker, Minneapolis, Minn.

ROMNEY, GEORGE-English; 1734-1802. Studied under Steele, also in Italy. In 1775 he established himself in London, where he divided public patronage with Reynolds and Gainsborough.

\section{The Widow.}

Loaned by T. B. Walker, Minneapolis, Minn.

\section{LELY, SIR PETER-(See 1.)}

\section{Miss Cavendish.}

Loaned by T. B. Walker, Minneapolis, Minn.

LORRAINE, CLAUDE-Born in Champagne on the Moselle in 1600 ; died in Rome, 1682. The most famous Landscape Artist of the 17 th century.

\section{Classic Harbor Scene.}

Loaned by T. B. Walker, Minneapolis, Minn.

REMBRANDT VAN RIJN-The greatest painter and etcher of the Dutch school. Born in Leyden, 1606; buried in Amsterdam, 1669. Pupil of Jacob Van Swanenburch, in Leyden, and, in 1623, of Pieter Lastman in Amsterdam. In spite of discouraging financial reverses Rembrandt worked steadily for about forty years.

43. Portrait of Himself.

Loaned by T. B. Walker, Minneapolis, Minn.

KNELLER, SIR GODFREY-Born at Lubeck, 1646; died at Twickenham, 1723. Portrait Painter; reported to have studied under Rembrandt and Bol, at Amsterdam, and in Rome (1672-74) under Carlo Maratti. He was induced to go to England in 1674. After the death of Sir Peter Lely he was made Court Painter.

44. Nell Gwyn.

Loaned by T. B. Walker, Minneapolis, Minn.

TURNER, J. M. W.-The most famous of English landscape painters. Born in London, 1775; died, 1851. Entered Royal Academy as student, 1789. Associate, 1799; Academician, 1802.

45. Venice.

Loaned by T. B. Walker, Minneapolis, Minr.

MILLET, JEAN FRANCOIS-(See 6.)

46. A Peasant of Brittany.

Loaned by J. B. Haggin, New York. 


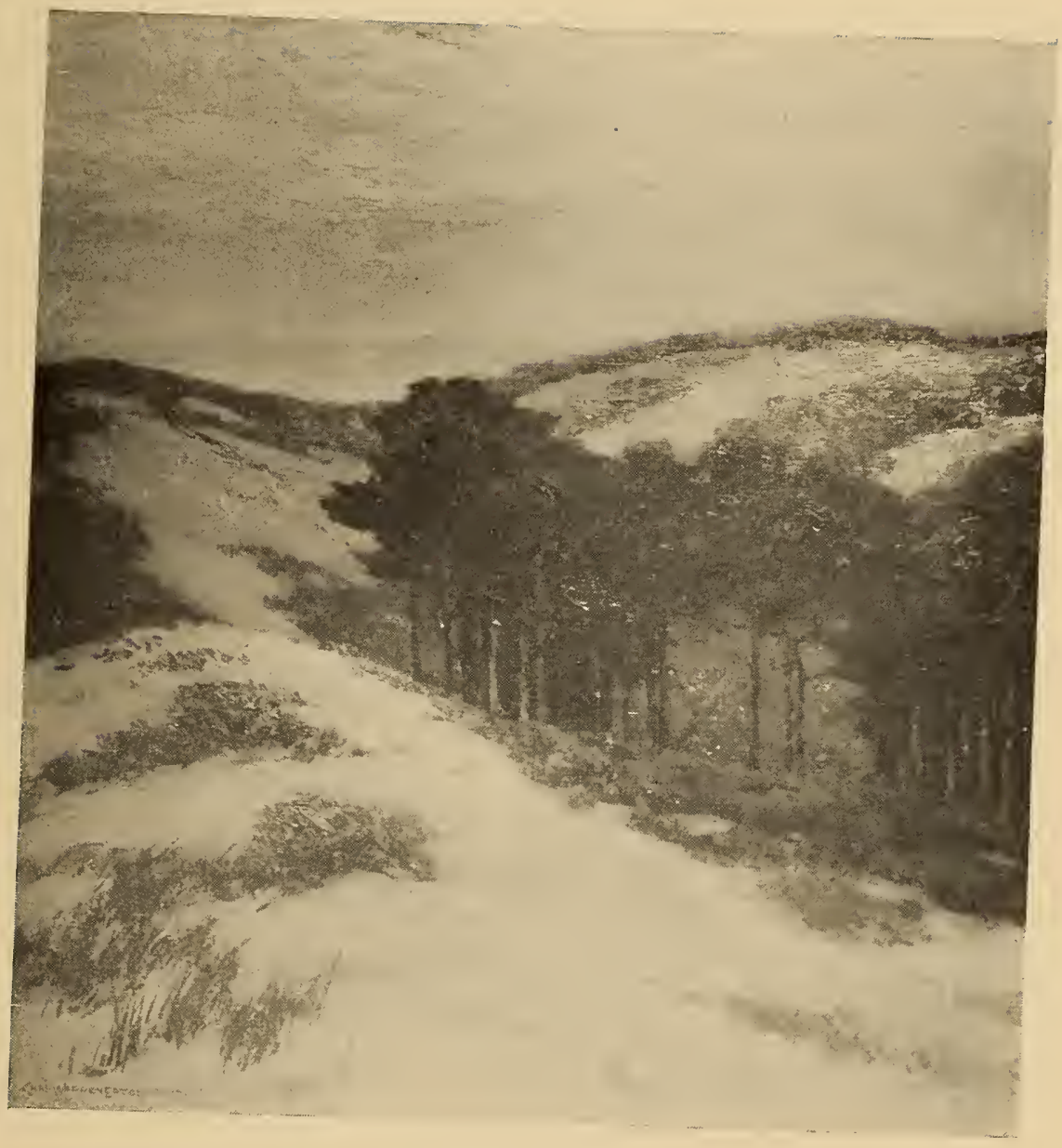

THE DUNES

Chas. Warren Eaton 

VAN DYCK, SIR ANTHONY-Born at Antwerp, 1599; died in England, 1641. Pupil of Rubens. He is chiefly distinguished as a portrait painter, although he executed many historical paintings.

\section{Mother and Child.}

Loaned by T. B. Walker, Minneapolis, Minn.

\section{Duke of Strafford.}

CORREGGIO (Antonio Allegri)-Born at Correggio in 1494; died there, March 5, 1534. Probably pupil of his father's brother, Lorenzo, and of Antonio Bartolotta, both second-rate painters of his native town. At Modena he is said to have found a better master in Francesco Bianchi, called Ferrari, who belonged to the school of Francia; but as Bianchi died in 1510, this, like all that concerns Correggio's training, is uncertain. Sweetness of expression and in-effable grace of pose characterize his works. His chiaroscuro has been praised by artists as simply perfect; it sheds a wonderful atmosphere of light and delight over all.

49. Virgin and Infant Jesus.

Loaned by T. B. Walker, Minneapolis, Minn.

KNELLER, SIR GODFREY-(See 44.)

50. Duchess of Marlborough.

Loaned by T. B. Walker, Minneapolis, Minn.

PHILIPPOTEAU, PAUL-French; 1815-1885. Became so well known as a Landscape and Battle Painter, that he was engaged by Americans to paint scenes on the Southern battle fields.

\section{Battle of Five Forks.}

Loaned by T. B. Walker, Minneapolis, Minn.

GAINSBOROUGH, THOMAS-English; 1727-1788. At fourteen years of age he went to London, where he studied under Hayman. He remained in London four years, acquiring much skill, and returned to his father's house a confirmed painter. In 1761 he made his debut at the Academy, and from this time until near the close of his life he was a regular contributor to the Academy Exhibitions. The combined grace and elegance of his portraits soon brought him into competition with Sir Joshua Reynolds.

\section{Gentleman in Blue.}

Loaned by T. B. Walker, Minneapolis, Minn.

HOPPNER, JOHN-English; 1758-1810. In 1775 he became a Pupil of the Royal Academy, and, by the patronage of the Prince of Wales, became a fashionable portrait painter, finding a rival only in Lawrence. Became in 1793 an A. R. A., and in 1795, R. A.

\section{Miss Moore.}

Loaned by The Ehrich Galleries, New York. 


\section{Gallery B}

MICHEL, GEORGES-Born at Paris, about 1763; died, 1S43. In his style he reverted to the sincerity and simplicity of the old Dutch Landscapists Hobbema and Ruisdael.

54. Landscape.

Loaned by H. T. Chapman, Brooklyn, N. Y.

ROUSSEAU, THEODORE-(See 16.)

55. Landscape Study.

Loaned by Francis Lathrop, New York.

NATTIER, JEAN MARC-French; 1685-1766. History and portrait Painter. Won the First Prize of the Academy in 1700. Member of the Academy in $171 \mathrm{~s}$, Adjunct Professor in 1746, and Professor in 1752. Associate member of the Copenhagen Academy.

56. Duchesse de Chateauroux and Comtesse de Flavacourt. Loaned by The Ehrich Galleries, New Iork.

CROME, JOHN (Called Old Crome)-English; 1769-1S21. He took to landscape painting without haring had any professional training, and settled at Norwich, where in 1805 he founded the Norwich Society of Artists.

57. Old Bridge near Norwich.

Loaned by H. T. Chapman, Brooklyn, N. Y

SCHREYER, ADOLPHE-(See 26.)

58. Rough Roads in Russia.

Loaned by Geo. A. Dowden, Newark, N. J.

WILSON, RICHARD-English; 1713-17S2. Father of English landscape and one of its greatest representatives. One of the orginal members of the Royal Academy.

59. Italian Landscape.

Loaned by Francis Lathrop, New York. 


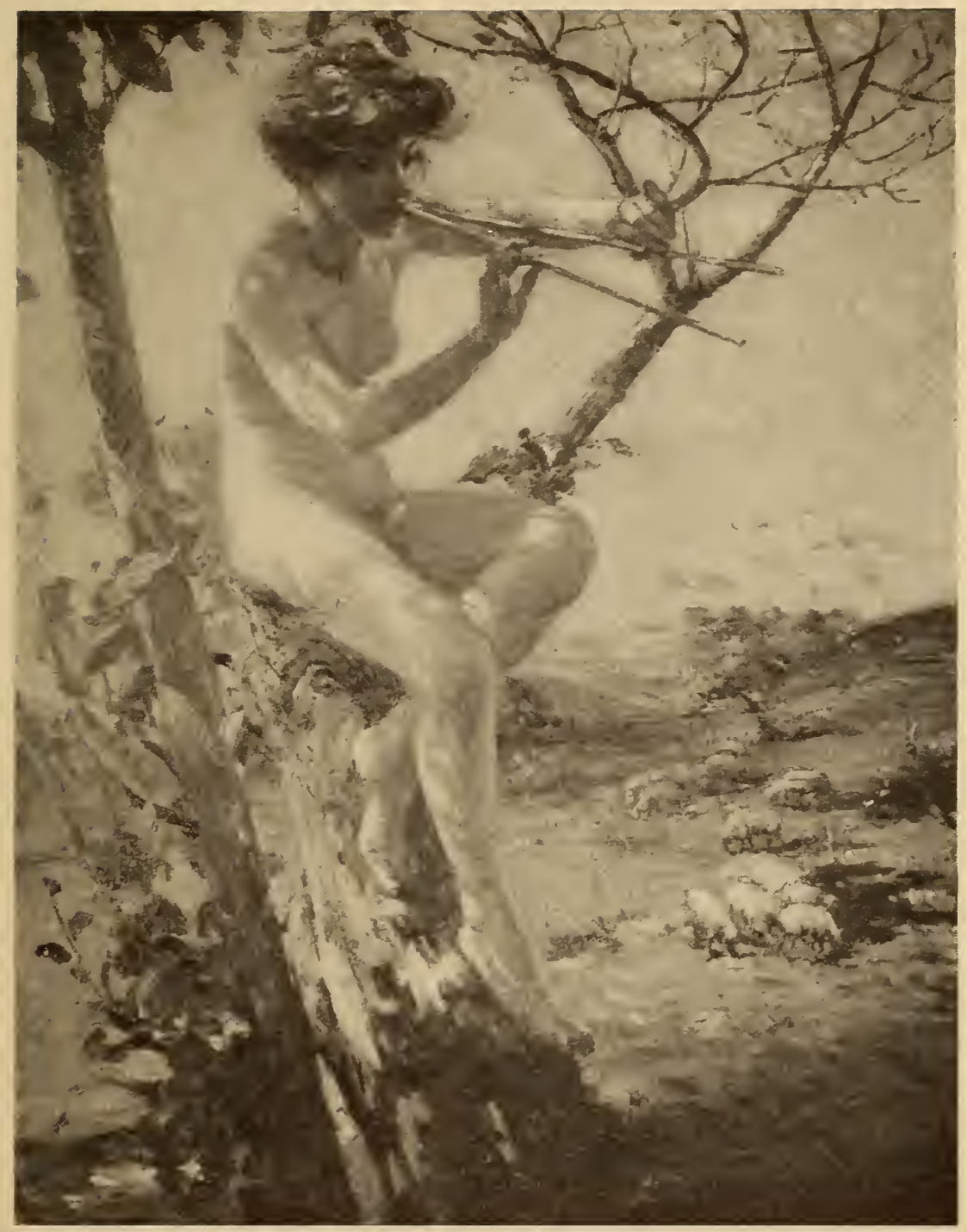

PASTORAI.

Lillian M. Genth. 

DIAZ, NARCISSE VIRGILE-(See 3.)

60. Figures.

Loaned by J. B. Haggin, New York.

REYNOLDS, SIR JOSHUA-English; 1723-1792. First President of the Royal Academy. Studied in London, under Hudson, and in Italy. Returned to London in 1752, and from 1760 onward his life was one unbroken success.

\section{Cupid Disarmed.}

Loaned by H. T. Chapman, Brooklyr, I. Y.

COROT, J. B. C.-(See 2.)

62. Landscape.

Loaned by J. B. Haggin, New York.

VIBERT, JEHAN GEORGES-French; 1840-1902. Pupil of Picot and Barrias. Medals, Salon, 1864, 1867, 1868; E. U., 1878. Chevalier Legion d'Honneur, 1870; Officier, 1882.

63. Four O'clock Tea.

Loaned by J. B. Haggin, New York.

ROMNEY, GEORGE-(See 40.)

64. Lady Hamilton as Meditation.

Loaned by Geo. A. Hearn, New York.

GAINSBOROUGH, THOMAS-(See 52.)

65. Girl Tending Pigs.

Loaned by Francis Lathrop, New York.

WATTS, GEORGE FREDERICK-English; 1817-1904.

66. Portrait of Joachim.

Loaned by Chas. L. Hutchinson, Chicago.

MICHEL, GEORGES-(See 54.)

67. Landscape.

Loaned by S. M. Vose, Providence, R. I.

TENIERS, DAVID (The younger)-Flemish school; 1610-1690. This characteristic Flemish landscape was painted conjointly by David Teniers, the younger, and Ambrois Breughel (1617-1675). The landscape is from the hand of Breughel and the figures were put in by Teniers.

68. Flemish Landscape.

Loaned by The Ehrich Galleries, New York. 
RICHTER, EDOUARD-Born in Paris. Genre and portrait painter. Pupil of Hebert and Bonnat.

69. Visitors of the Louvre, Paris.

Loaned by Mark Arnheim, New York.

COUTURE, THOMAS-French; 1815-1879. Portait and historical painter. Pupil of Gros and Delarche Medals 1844, 1847, 1855. Chev. Legion d'Honneur, 1848.

70. St. Jerome.

Loaned by Geo. A. Hearn, New York.

DUPRE, JULES-(See 10.)

71. Landscape.

Loaned by J. B. Haggin, New Yorik.

DE WINT, PETER-English; 1784-1849.

72. Near Wilford, Lincolnshire.

Loaned by The Blakeslee Galleries, New York.

CARRACCI, LUDOVICO-Born in Italy, 1555; died, $1617 . \mathrm{He}$ was the real founder of the famous Bolognese School, and stands out as one of the greatest Italian masters after the early Renaissance period.

73. Susanna and the Elders.

Loaned by The Ehrich Galleries, New York.

COURBET, GUSTAVE-French; 1819-1877. Genre, landscape and portrait painter. A leader of the realists. Pupil of David d'Angers.

74. La Geante des Montagnes du Doubs.

Loaned by H. T. Chapman, Brooklyn, N. Y.

DL ?RE, JULES-(See 10.)

75. Landscape.

Loaned by Charles E. Bushnell, New York.

TINTORETTO, IL-Venetian school. Born, 1518; died, 1594.

76. Mother and Child.

Loaned by Francis Lathrop, New York. 


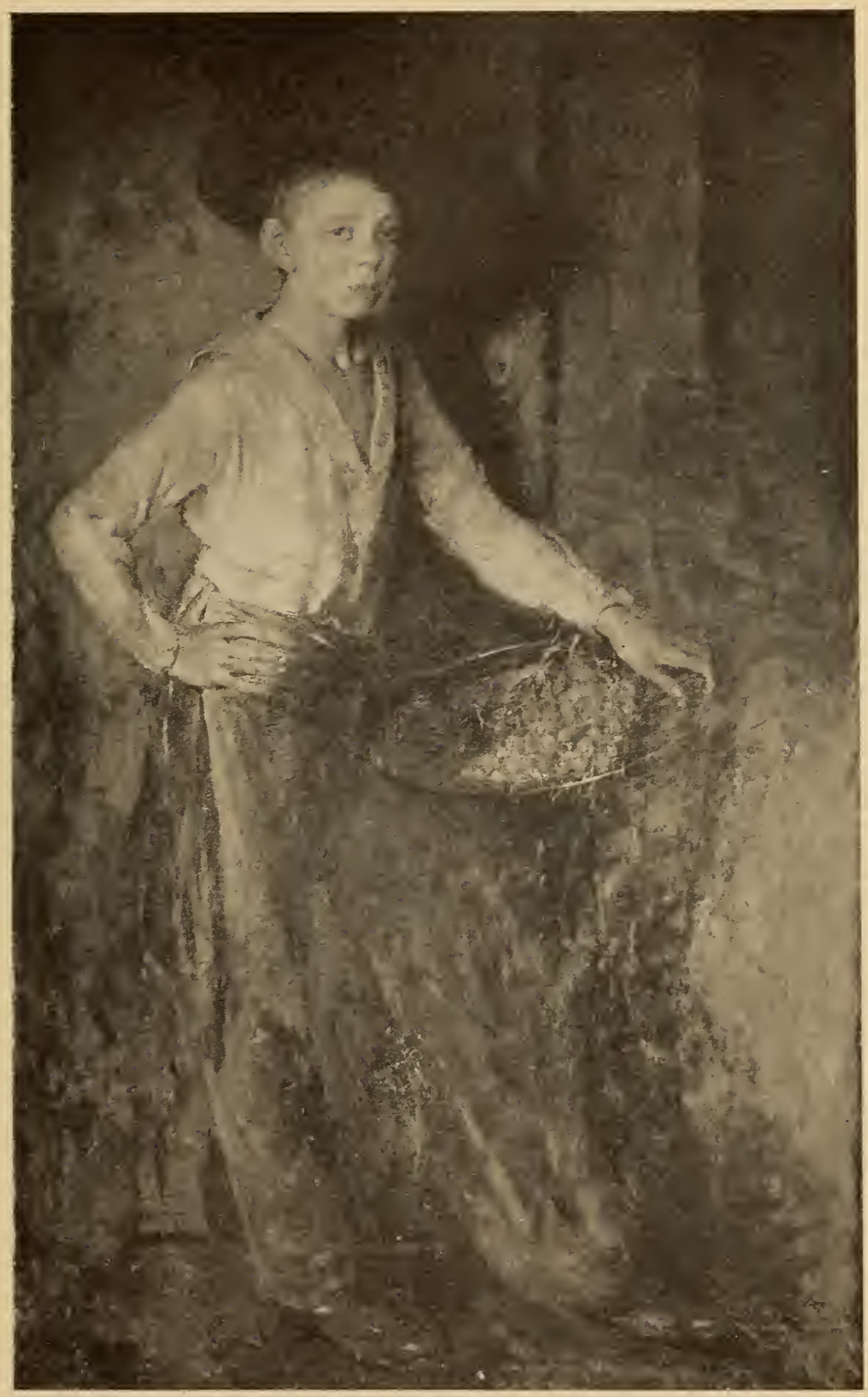

BOY WITH GRAPES

Charles $W$. Hawthorne 

MORLAND, GEORGE-(See 19.)

77. The Red Lion Inn.

Loaned by The Ehrich Galleries, New York.

LAWRENCE, SIR THOMAS-English; 1769-1830. His first efforts were chalk portraits of his father's customers. In 1787 he settled in London and entered as a student in the Royal Academy. Elected A. R. A. in 1791, though under the required age. Succeeded West as Painter to the King. R. A. in 1794. Knighted in 1815; P. R. A., 1820; Chev. Legion d'Honneur, France, 1825.

\section{Lady Blessington.}

Loaned by The Ehrich Galleries, New York.

HUDSON, THOMAS-Devonshire; 1701-1779. Hudson was Reynold's master; his works are rare.

79. Lady Nugent.

Loaned by S. M. Vose, Providence, R. I.

ZIEM, FELIX-(See 9.)

80. Evening Glow in Venice.

Loaned by Geo. A. Dowden, Newark, N. J.

GAINSBOROUGH, THOMAS-(See 52.)

81. Miss Margaret Burr.

Loaned by Francis Lathrop, New York.

DIAZ, NARCISSE VIRGILE-(See 3.)

82. The Quarry.

Loaned by Francis Lathrop, New York.

DELACROIX, F. V. E.-(See 25.)

83. Horsemen.

Loaned by Francis Lathrop, New York.

RUBENS, PETER PAUL-Head of the Flemish school of the 17 th century, and the greatest historical painter north of the Alps. Born, 1577; died, 1640 .

84. Christ Delivering the Keys to Peter.

Loaned by Geo. A. Hearn, New York. 
DAUBIGNY, CHARLES-FRANCOIS-French; 1817-1878. Pupil of Delaroche. Spent three years in Italy. Medals of Salon, 1848, 1853, 1855, 1857 (Rappel,) 1859 (Rappel.) E. U., 1855, 1867. Chev. Legion d'Honneur, 1859; Officier, 1874. Diploma to Memory of Deceased Artists, 1878.

85. Landscape, on the French Coast.

Loaned by J. B. Haggin, New York.

MILLET, JEAN FRANCOIS-(See 6.)

86. Flight into Egypt.

Loaned by S. M. Vose, Providence, R. I.

BATTONI, POMPEO GIROLAMO-Italian School; born in Lucca, 1708 ; died, 1787.

87. The Singing Canon.

Loaned by The Blakeslee Galleries, New York.

MURILLO, BARTHOLOME ESTEBAN-Spanish; 1618-1682. Studied under Juan del Castillo. All his works are distinguished by a close and lively imitation of nature; there is little of the academy discernible in his design or his composition.

88. The Nativity.

Loaned by The Ehrich Galleries, New York.

DAUBIGNY, CHARLES FRANCOIS-(See 85.)

89. On the Oise.

Loaned by Geo. A. Dowden, Newark, N. J.

TURNER, J. M. W.-( See 45.)

90. Landscape, in the Tropics.

Loaned by J. B. Haggin, New York.

MOREELSE, PAUL-Dutch School; born in Utrecht, 1571; died, 1638 .

91. Portrait of Lady.

Loaned by J. B. Haggin, New York.

POTTER, PAUL-Dutch; born in Enkhuigen, 1625; died, 1654. Landscape and animal painter.

92. Cattle on the Alert.

Loaned by Mark Arnheim, New York. 


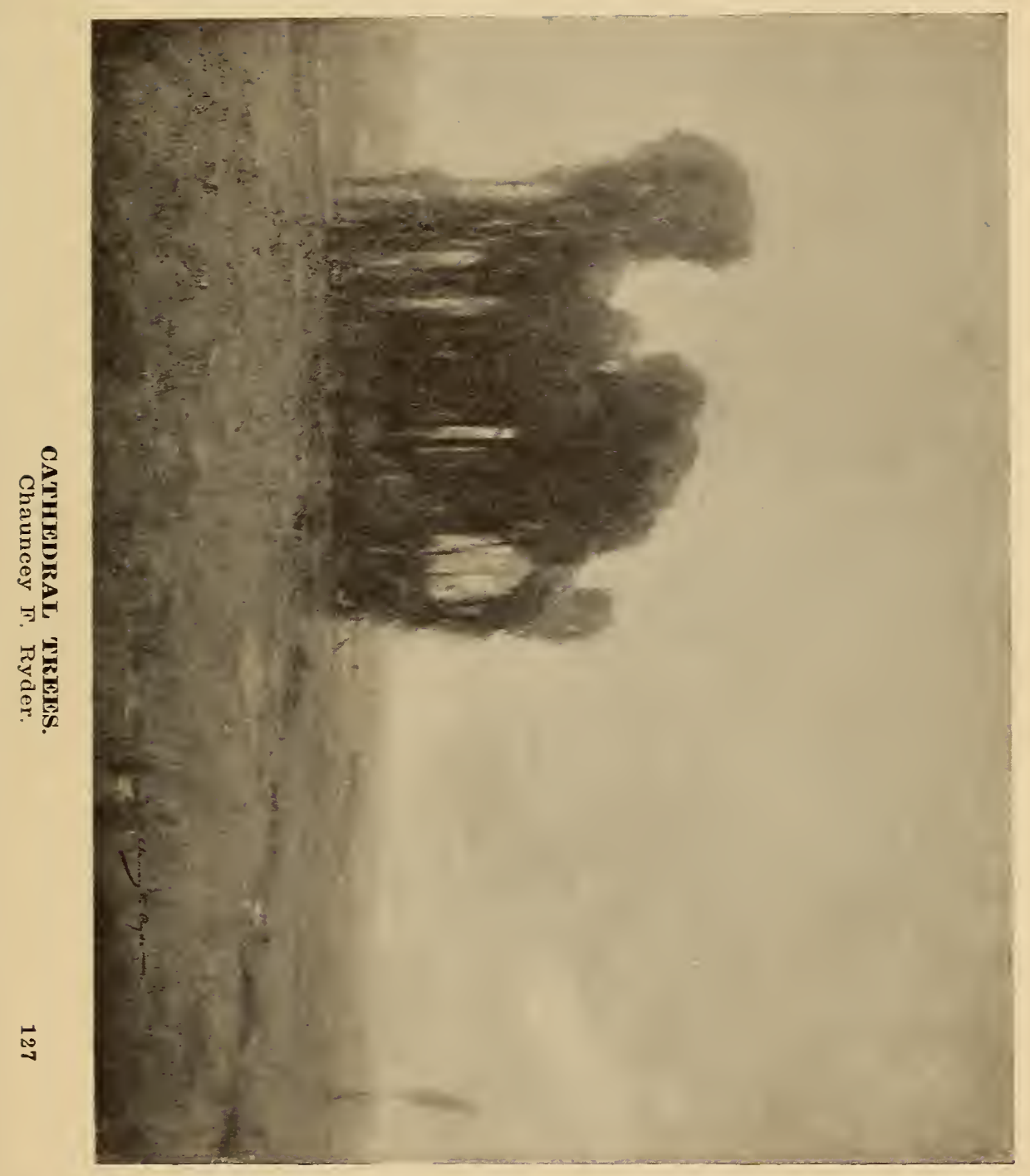



DAUBIGNY, CHARLES FRANCOIS-(See 85.)

\section{Approaching Sunset.}

Loaned by The Schaus Galleries, New York.

POURBUS, FRANS (The younger)-Born at Antwerp, 1570; died at Paris in 1622. A skillful painter of portraits and history.

94. Anne of Austria.

Loaned by The Blakeslee Galleries, New York.

\section{DIAZ, NARCISSE VIRGILE-(See 3.)}

\section{The Lovers.}

Loaned by H. T. Chapman, Brooklyn, N. Y.

COROT, J. B. C.-(See 2.)

96. Landscape.

Loaned by Geo. A. Dowden, Newark, N. J.

MYTENS, DANIEL (The elder)-Dutch portrait painter; born at The Hague at the end of the $16 \mathrm{th}$ centry, died after $1658 . \mathrm{He}$ probably studied under Rubens. Went to England during the latter part of the reign of King James; was employed as Court Painter by Charles I, but returned to The Hague somietime after the arrival of Van Dyck.

97. Charles I.

Loaned by The Ehrich Galleries, New York.

LEYS, BARON HENRI-Born at Antwerp, 1815; died, 1869. Medal, Paris, 1846; Legion of Honor, 1847; Grand Medal of Honor, 1855, (Universal Exhibition); one of the eight Grand Medals of Honor, Universal Exhibition, 1867; Created Baron, 1862; Knight of the Order of Leopold. Diploma to the Memory of Deceased Artists, 1878.

\section{Rembrandt's Studio.}

Loaned by Chas. L. Hutchinson, Chicago.

VIBERT, J. G.-(See 63.)

99. Pleased with Himself.

Loaned by Geo. A. Dowden, Newark, N. J.

REYNOLDS, SIR JOSHUA-(See 61.)

100. Archbishop of York.

Loaned by The Blakeslee Galleries, New York. 


\section{Lower Atrium}

PICKNELL, WILLIAM L.-Born Vermont, 1852; died, 1897. Studied for two years in Rome under Inness; later, for a few months under Gerome, in Paris. From France he went to England, and for a number of years was an exhibitor at the Royal Academy.

101. Squam, Mass.

Loaned by Wm. S. Hurley, Brooklyn, N. Y.

MORA, F. LUIS-Born Montevideo, Uruguay, 1874. Pupil of School of Boston Museum under Benson and Tarbell; A. S. L., of N. Y., under Mowbray. Gold Medal, A. C. P., 1901; Gold Medal, A. A. S., 1902; Two Bronze Medals, St. Louis Exposition, 1904; First Hallgarten Prize, N. A. D., 1905 ; Evans Prize, Salmagundi Club, 1908 ; Beal Prize, W. C. S., 1908; A. N. A., 1904; A. N., 1906 ; A. S. L., of New York, Salmagundi Club, 1904. Also Teacher.

\section{American Gladiators.}

BONNAT, LEON-Born at Bayonne, 1833. Pupil in Madrid of F. de Madrazo, and of Cogniet, in Paris. Studio in Paris. Second Grand Prix, 1858; Then Studied Three Years in Italy. Medals; Second Class, 1861, 1863, and 1869; of Honor, 1869. Legion of Honor, 1867; Officer, 1874; Commander, 1882; Grand Officer, 1897. Member of the Institute of France.

\section{Sampson and the Lion.}

Loaned by Pacific Trust Co., Seattle.

HAGGIN, BEN. ALI-Third Hallgarten prize, N. A. D., 1909. 104. Mary Garden-“Thais."

WAUGH, FREDERICK J.-Philadelphia, Pa. Member A. C. P. 105. Under the Full Moon. 


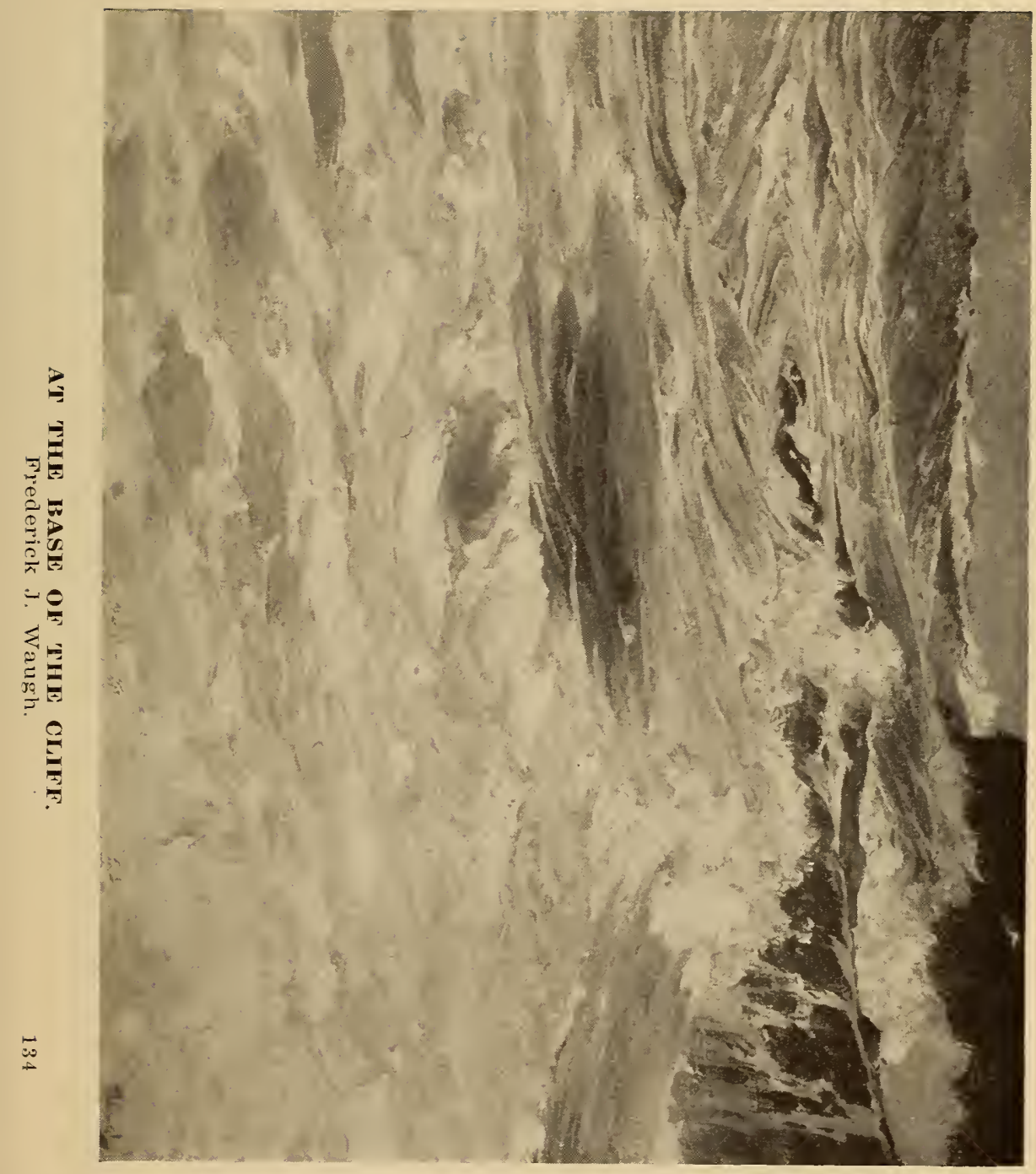





\section{Gallery C}

TWACHTMAN, JOHN HENRY-Born in Cincinnati, O., 1853; died, 1902. Studied at the School of Design under Frank Duvenick and in Munich and Paris. Medal, Columbia Exposition, Chicagc, 1893; Webb Prize, S. A. A.; Temple Gold Medal, P. A. F. A., 1895; Silver Medal, Pan-American Exposition, Buffalo, 1901. Member S. A. A., 1879; Ten American Painters.

\section{Greenwich Hills.}

Loaned by Silas S. Dustin, New York.

ALMA-TADEMA, SIR LAURENZ-Born in Holland, 1836; lived in London since 1870. Studied in Antwerp, and afterwards with Baron Leys. Paris Medals (Salon) 1864, (E. U.) 1867, 1878, 1889, 1900 (Grand Prix). Officer Legion d'Honneur, 1878. R. A., London.

107. The Patriarch.

Loaned by Geo. A. Dowden, Newark, N. J.

EATON, CHARLES WARREN-Born Albany, N. Y., 1857. Pupil of N. A. D., and A. S. L. of N. Y. Honorable Mention, Paris Exposition, 1900; Proctor Prize, Salmagundi Club, 1901; Honorable Mention, Pan-American Exposition, Buffalo, 1901; Silver Medal, Charleston Exposition, 1902; Inness Prize, Salmagundi Club, 1902; Shaw Prize, Salmagundi Club, 1903; Gold Medal, A. C. P., 1903; Inness Gold Medal, N. A. D., 1904; Silver Medal, St. Louis Exposition, 1904; Medal (Third Class) Paris Salon, 1906; A. N. A., 1901; Member A. W. C. S.; N. Y. W. C. C.; Salmagundi Club, 1897; A. Fund S.; Specialty, Landscapes.

\section{The Dunes.}

LOW, WILL H.-Born Albany, N. Y., 1853. Pupil of Ecole des Beaux Arts under Gerome, and of Carolus-Duran in Paris. Silver Medal, Paris Exposition, 1889; Medal, Columbian Exposition, Chicago, 1893; Lotus Club, Fund, N. A. D., 1895; Silver Medal, Pan-American Exposition, 1901; Member International Jury of Awards, St. Louis Exposition, 1904. Member N. A., 1890, (Res. Sec.); S. A. A., 1878; Mural P.; New York Arch. Lg., 1889; Century Association. Also Writer.

109. Her First Communion. 
GENTH, LILLIAN MATILDE-Born Philadelphia, July 6, 1876. Pupil of Elliott Daingerfield in New York; James McN. Whistler in Paris. Mary Smith Prize, P. A. F. A., 1904; Julia Shaw Prize, N. A. D., 1908. Association Member Fellowship, P. A. F. A.; A. N. A., (elect) 1909.

110. Pastoral.

DESSAR, LOUIS PAUL-Born Indianapolis, Ind., 1867. Pupil of N. A. D., Bouguereau, Robert-Fleury and Ecole des Beaux Arts in Paris. Third Class Medal, Salon, 1891; Medal, Columbian Exposition, Chicago, 1893; Honorable Mention, C. I., Pittsburg, 1897; Second Hallgarten Prize, N. A. D., 1900; Bronze Medal, Paris Exposition, 1900; Silver Medal, Pan-American Exposition, Buffalo, 1901; Silver Medal, Charleston Exposition, 1902; A. N. A., 1899; N. A., 1906; Member S. A. A., 1898; Salmagundi Club, 1895; A. Fund S.

111. Dolly and Beckey.

Loaned by The Folsom Co., New York.

VAN LAER, ALEXANDER T.-Born Auburn, N. Y., Feb. 9, 1857. Pupil of N. A. D. and R. Swain Gifford in New York; Geo. Poggenbeek in Holland. Bronze Medal, Charleston Exposition, 1902; Member International Jury of Awards, St. Louis Exposition, 1904; A. N. A., 1901; Member Lotos C. (life); Salma. C., 1892 (pres.); A. Fund S. Specialty, Landscapes. Also teacher, writer and lecturer.

112. January Afternoon.

PATERSON, JAMES-Glasgow school. Member of the Royal Scottish Academy; Royal Society of Painters in Water Colors, Glasgow, and the International Society of Sculptors, Painters and Gravers, London; Corresponding Member of the Secession, Munich. Honorable Mention, Salon, 1890; Medal of the Second Class, Glaspalast, Munich, 1890.

\section{The Dell, East Lynton.}

Loaned by The Albright Art Gallery, Buffalo.

THAULOW, FRITZ-Born in Christiania, Norway, 1847; died, Nov. 5, 1907. Studied in Christiania, Carlsruhe and Paris. Gold Medals at Vienna and Munich. Diploma of Honor, Dresden. Member of Jury at Centennial Exhibition. Philadelphia, 1876; and at Carnegie Institute, Pittsburg, 1898. Chevalier Legion d'Honneur, 1889; of the Order of Leopold, Belgium; and St. Olaf, Norway. Grand Prix, E. U., Paris, 1900.

\section{Verona from the Old Bridge.}

Loaned by Charles E. Bushnell, New York.

HAWTHORNE, CHARLES W.-Born in Illinois. Pupil of Wm. M. Chase and $H$. Siddons Mowbray in New York. Obrig Prize, Salmagundi Club, 1902; First Hallgarten Prize, N. A. D., 1904; Evans Prize, Salmagundi Club, 1904; Shaw Purchase Prize, Salmagundi Club, 1904; Second Prize, Worchester, Mass,, 1904; Second Hallgarten Prize, N. A. D., 1906. Member Salmagundi Club, 1900 ; A. Fund S.; A. N. A. (elect.) Also Teacher.

115. Boy with Grapes.

TWACHTMAN, JOHN H.-(See 106.)

116. Spring Morning.

Loaned by Silas S. Dustin, New York. 


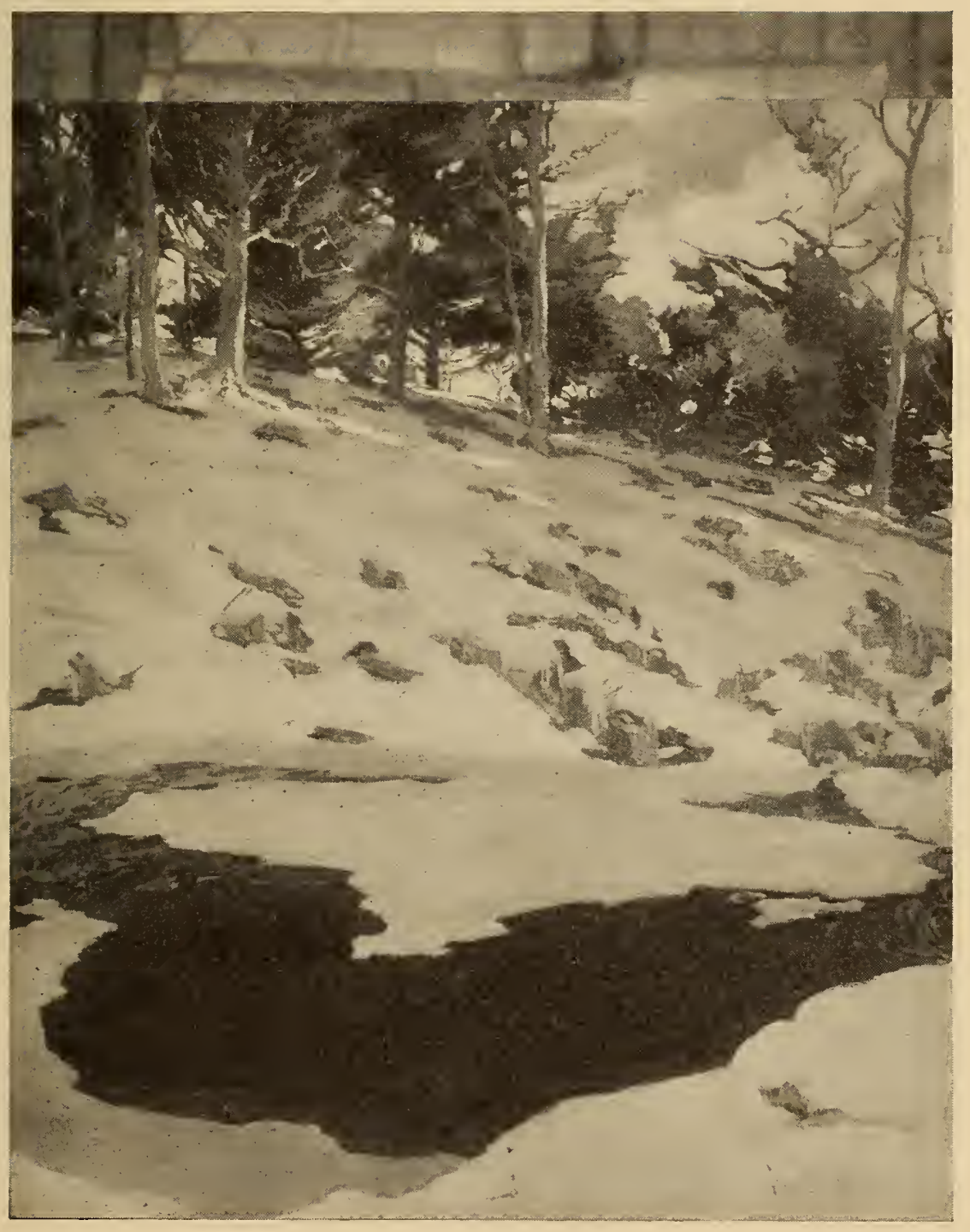

THE WITCHES POOL.

Jonas Lie. 

HARRISON, L. BIRGE-Born in Philadelphia, Pa., 1854. Pupil of Cabanel in Paris. Silver Medal, Paris Exposition, 1889; Medal, Columbian Exposition, 1893; Bronze Medal, Pan-American Exposition, Buffalo, 1901; Second Corcoran Prize, S. Washington A., 1904; Silver Medal, St. Louis Exposition, 1904. "Hors Concours," Paris Salon, A. N. A., 1902; Member S. A. A., 1882; Fellowship, P. A. F. A.; Century Association. Specialty, Landscapes.

\section{Flatiron in a Blizzard.}

BENSON, FRANK W.-Born Salem, Mass., March 24, 1862. Pupil of Museum of Fine Arts in Boston; Boulanger and Lefebvre in Paris. Third Hallgarten Prize, N. A. D., 1889; Clarke Prize, N. A. D., 1891; Medal, Columbian Exposition, Chicago, 1893; Medal Mechanics Assoc., Boston; Ellsworth Prize, A. I. C.; Cleveland Art Assoc. Prize; Jordan Prize, Boston, $\$ 500$ in 1894 and $\$ 300$ in 1895; B. A. C. Prize, $\$ 1,000$ in 1895 and $\$ 100$ in 1896 ; Shaw Prize $(\$ 1,500)$, S. A. A., 1896; Chronological Medal, C. I., Pittsburg, 1896; Second Class Medal $(\$ 1,000)$, C. I., Pittsburg, 1899; Silver Medal, Paris Exposition, 1900; Silver Medal, Pan-American Exposition, Buffalo, 1901; Lippincott Prize, P. A. F. A., 1903; Gold Medal ( $\$ 1,500)$, C. I., Pittsburg, 1903; Two Gold Medals, St. Louis Exposition, 1904; Proctor Prize, N. A. D., 1906; Gold Medal, A. C. P., 1906; Second Prize, Corcoran Gallery, 1907; Temple Gold Medal, P. A. F. A., 1909. N. A., 1905; Member Ten American Painters; Nat. Inst. A. L. Also Teacher.

118. October, Decorative Panel.

TRYON, DWIGHT WILLIAM-Born Hartford, Conn., Aug. 13, 1849. Pupil of C. Daubigny, Jacquesson de la Chevreuse, A. Guillemet and $H$. Harpignies in Paris. Bronze Medal, Boston, 1882; Gold Medals, American Art Assoc., New York. 1886 and 1887; Third Hallgarten Prize, N. A. D., 1887; Ellsworth Prize, A. I. C., 1888; Palmer Prize, Chicago Interstate Exposition, 1889; Webb Prize, S. A. A., 1889; First Class Gold Medal, Munich International Exposition, 1892; Medal, Columbian Exposition, Chicago, 1893; First Prize, Cleveland Interstate Exposition, 1895; First Prize, Tennessee Centennial, 1897; Gold Medal $(\$ 1,500)$, C. I., Pittsburg, 1898; Chronological Medal, C. I., Pittsburg, 1899; Gold Medal, Pan-American Exposition, Buffalo, 1901; Gold Medal, St. Louis Exposition, 1904. Member N. A., 1891; S. A. A., 1882; A. W. C. S. Professor of Art, Smith College. Specialty, Landscapes.

119. Twilight at Arverne.

Loaned by Wm. T. Evans, Montclair, N. J.

CRANE, FREDERICK-Born Bloomfield, N. J., 1847. Bronze Medal, St. Louis Exposition, 1904; Member Salmagundi Club, 1901.

120. Long Island Coast.

COUSE, EANGER IRVING-Born Saginaw, Mich., 1866. Pupil of N. A. D., in New York; Bouguereau, Robert-Fleury, and Ecole des Beaux Arts in Paris. Shaw Prize for black and white at Salmagundi Club, 1899; Second Hallgarten Prize N. A. D., 1900; Proctor Prize, Salmagundi Club, 1900; Honorable Mention, Paris Exposition, 1900; Honorable Mention, Pan-American Exposition, Buffalo, 1901; First Hallgarten Prize, N. A. D., 1902; Two Bronze Medals, St. Louis Exposition, 1904; A. N. A., 1902; Member A. W. C. S.; Salmagundi Club, 1898; N. Y. W. C. C., 1902; Lotus Club (life.)

121. The Voice of the Falls. 
HASSAM, CHILDE-Born Boston, 1859. Pupil of Boulanger and Lefebvre in Paris. Bronze Medal, Paris Exposition, 1889; Gold Medal, Munich, 1892; Medal, A. C. P., 1892; Medal, Columbian Expositon, Chicago, 1893; Prize, Cleveland Art Association, 1893; Webb Prize, S. A. A., 1895; Prize, B. A. C., 1896; Second Class Medal, C. I., Pittsburg, 1898; Temple Gold Medal, P. A. F. A., 1899; Silver Medal, Paris Exposition, 1900; Gold Medal, Pan-American Exposition, Buffalo, 1901; Gold Medal, St. Louis Exposition, 1904; Clarke Prize, $\mathbf{N}$. A. D., 1905; Third Class Medal, C. I., Pittsburg, 1905; Lippincott Prize, P. A. F. A., 1906; Carnegie Prize, S. A. A., 1906; Third Prize, Worcester, 1906. Member A. W. C. S.; N. Y. W. C. C.; B. A. C.; Ten American Painters; A. N. A., 1902; N. A., 1906; Munich Secession (Cor.); Assoc. Nat. des Beaux Arts, Paris; Nat. Inst. A. L.

\section{The Blue Kimono.}

GROLL, ALBERT L.-Born New York, 1866. Pupil of Gysis and Loefftz in Munich. Morgan Prize, Salmagundi Club, 1903; Honorable Mention, Munich; Shaw Prize, Salmagundi Club, 1904; Silver Medal, St. Louis Exposition, 1904; Sesnan Medal, P. A. F. A., 1906. Member A. Fund S.; Country Sketch C.; Salmagundi Club, 1900; A. N. A., 1906. Specialty, Landscapes.

123. Laguna, New Mexico.

BECKWITH, J. CARROLL-Born Hannibal, Mo., Sept. 23, 1852. Pupil of Carolus-Duran and Ecole des Beaux Arts. Honorable mention, Paris Salon, 1887; Bronze Medal, Paris Exposition, 1889; Gold Medal, Atlanta Expositon, 1895; Bronze Medal, Paris Expositon, 1900; Gold Medal, Charleston Expositon, 1902; Member N. A., 1894; S. A. A., 1881; A. W. C. S.; A. S. L. (hon.) ; A. Fund S. ; Salma. C., 1901; Century Ass'n; N. Y. Art Commission; Metropolitan Museum; Nat'l Institute, A. L.

\section{The Awakening.}

JONES, FRANCIS C.-Born Baltimore, Md., 1857. Pupil of Boulanger and Lefebvre, Ecole des Beaux-Arts in Paris. Clarke Prize, N. A. D., 1885; Silver Medal, Pan-American Exposition, Buffalo, 1901; Shaw Purchase, S. A. A. 1904; Silver Medal, St. Louis Exposition, 1904; Member N. A., 1894; S. A. A., 1882; A. W. C. S.; N. Y. Arch. Lg., 1888; Mural P.; N. A. C.; A. Aid S.; Century Association. Also Teacher.

125. The Rose.

POTTHAST, EDWARD HENRY-Born Cincinnati, O., 1857. Pupil of Cincinnati School of Fine Arts; studied in Antwerp, Munich and Paris. Clarke Prize, N. A. D., 1899; Evans Prize, A. W. C. S., 1901; Gold Medal, A. A. S., 1902; Silver Medal, St. Louis Exposition, 1904 ; A. N. A., 1899; N. A., 1906; Member S. A. A., 1902; A. W. C. S.; N. Y. W. C. C.; Salmagundi Club, 1895; Nat. Inst. A. L.

126. Hazy October Day.

RYDER, CHAUNCEY F.-Born Danbury, Conn., 1868. Pupil of A. I. C.; Julian Academy, Collin, Laurens and Max Bohm in Paris. Honorable Mention, Paris Salon, 1907.

127. Cathedral Trees. 


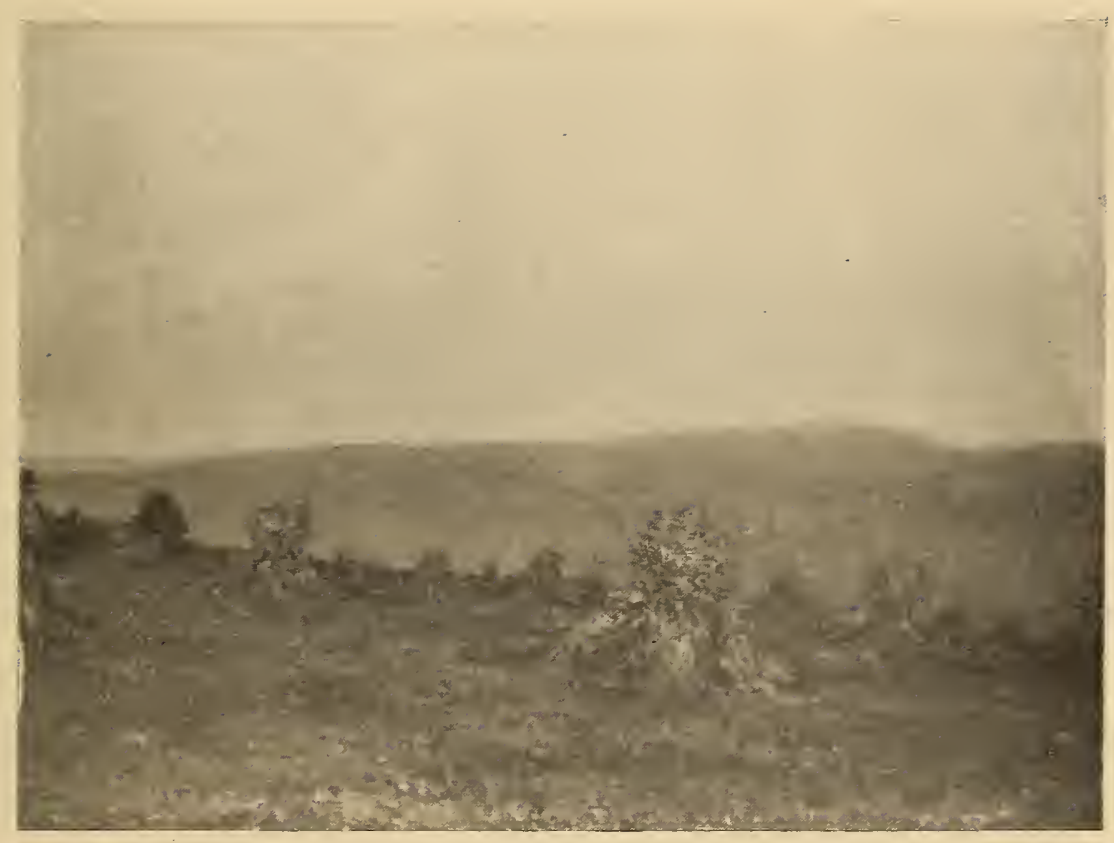

RYE FIELD

Cullen Yates 

HENRI, ROBERT-Born Cincinnati, Ohio, 1865. Pupil of P. A. F. A.; Julian Academy and Ecole des Beaux Arts in Paris. Silver Medal, Pan-American Exposition, Buffalo, 1901; Silver Medal, St. Louis Exposition, 1904; Harris Prize, A. I. C., 1905. Member S. A. A., 1903; Eight American Painters; A. N. A., 1905; N. A., 1906; Fellowship, P. A. F. A.

128. Girl with Plumes.

WEIR, JULIAN ALDEN-Born West Point, N. Y., Aug. 30, 1852. Pupil of his father, Robert W. Weir; Gerome in Paris. Honorable Mention, Paris Salon, 1882; Silver Medal for Painting and Bronze for Drawing, Paris Exposition, 1889; Medal C. I., Pittsburg, 1897; Prize $(\$ 2,000)$, American Art Assoc., New York; Bronze Medal, Paris Exposition, 1900; Gold Medal, Pan-American Exposition, Buffalo, 1901; Gold Medal for Paintings and Silver Medal for Engravings, St. Louis Exposition, 1904; Inness Gold Medal, N. A. D., 1906. Member N. A., 1880; A. W. C. S.; N. Y. Etching C.; A. Aid S.; Century Assoc.; Nat. Inst. A. I.

129. Windham Landscape.

DAVIES, ARTHUR B.-Born Utica, N. Y., 1862. Silver medal Pan-American Exposition, 1901; Member N. Y. W. C. C.; Eight American Painters.

130. In the High Sierras.

Loaned by William Macbeth, New York.

WEISS, JOSE-Contemporary. Exhibited in 1908 at the Royal Academy, London; and the Glasgow Institute of Fine Arts.

131. Spring Floods.

Loaned by R. C. \& N. M. Vose, Boston.

DOMINGO, J.-Born in Spain. Pupil of Meissonier in Paris.

132. Halt by the Wayside.

Loaned by Geo. A. Dowden, Newark, N. J.

COFFIN, WILLIAM ANDERSON-Born Allegheny, Pa., 1855. Pupil of Bonnat in Paris. Second Hallgarten Prize, N. A. D., 1886; S. A. A. Webb Prize, 1891; Bronze Medal, Paris Exposition, 1889; Director of Fine Arts and member of the Superior Jury, Pan-American Exposition, Buffalo, 1901; Silver Medal, Charleston Exposition, 1902 ; Silver Medal, St. Louis Exposition, 1904; A. N. A., 1899; Member S. A. A., 1886; S. Landscape P.; New York Arch. Lg., 1888. Specialty, Landscape. Writer on Art.

133. Sunrise in Winter.

WAUGH, FREDERICK J.-(See 105.)

134. At the Base of the Cliff.

SCHRAM, A. S.

135. At the Shrine of Eros.

Loaned by Geo. A. Dowden, Newark, N. J. 
FOSTER, BEN.-Born North Anson, Maine. Pupil of Abbott H. Thayer in New York, Morot and Merson in Paris. Medal, Columbian Exposition, Chicago, 1893; Second Prize, Cleveland, 1895; Bronze Medal, Paris Exposition, 1900; Silver Medal, C. I., Pittsburg, 1900; Silver Medal, Pan-American Exposition, Buffalo, 1901; Webb Prize, S. A. A., 1901; Silver Medal, St. Louis Exposition, 1904; Carnegie Prize, N. A. D., 1906; Inness Gold Medal, N. A. D., 1909; A. N. A., 1901; N. A., 1904; Member S. A. A., 1897; N. Y. W. C. C.; Century Association; Specialty, Landscapes; Writer on Art.

136. October End.

HASSAM, CHILDE-(See 122.)

137. June Morning, Sunlight Through the Leaves.

HUGUET, VICTOR PIERRE-French; 1835-1902.

138. Chevaux a l'abreuvoir.

Loaned by Durand-Ruel \& Sons, New York.

KNAPP-Early American.

139. William Henry Seward.

Loaned by Wm. S. Seward, Auburn, N. Y.

MELCHERS, J. GARI-Born Detroit, Mich., 1860. Pupil of Lefebvre and Boulanger in Paris. Honorable Mention, Paris Salon, 1886; First Class Medal, Amsterdam, 1887; Third Class Medal, Paris Salon, 1888; First Class Medal, Munich, 1888; Grand Prize, Paris Exposition, 1889; First Prize, A. I. C., 1891; Medal of Honor, Berlin, 1891; Gold Medal, A. C. P., 1892; Medal of Honor, Antwerp, 1894; Temple Gold Medal, P. A. F. A., 1896; First Class Medal, Vienna, 1898; Gold Medal, Pan-American Exposition, Buffalo, 1901; Knight of the Order of St. INicheal of Bavaria; Chevalier of the Legion of Honor of France; Officer, 1904; Gold Medal, St. Louis Exposition, 1904; Member Paris S. A. P.; Soc. Nat'l des Beaux Arts; International Society of Artists, London; Munich Secession (Cor.); A. N. A., 1904 ; N. A., 1906 ; Nat. Inst. A. L.

140. The Arbor.

LIE, JONAS-Born in Norway, 1880. Pupil of N. A. D. and A. S. L., of N. Y. Silver Medal, St. Louis Exposition, 1904. Specialty, Landscapes.

\section{The Witches' Pool.}

DAVIS, CHARLES H.-Born Amesbury, Mass., 1856. Pupil of Otto Grundmann and Museum of Art in Boston; Boulanger and Lefebvre in Paris. Gold Medal, American Art Assoc., New York, 1886; Honorable Mention, Paris Salon, 1887; $\$ 2,000$ Prize, American Art Assoc., New York, 1887; Silver Medal, Paris Exposition, 1889; Palmer Prize, A. I. C., 1890; Medal Mass. Charitable Mechanics Assoc., Boston, 1890; Medal, Columbian Exposition, Chicago, 1893; Grand Gold Medal, Atlanta Exposition, 1895; Bronze Medal, Paris Exposition, 1900; Lippincott Prize, P. A. F. A., 1901; Silver Medal, Pan-American Exposition, Buffalo, 1901; Second Corcoran Prize, S. Washington A., 1902; Silver Medal, St. Louis Exposition, 1904. Member S. A. A., 1886; A. N. A., 1901; N. A., 1906; Copley S. (VicePres.)

142. Oak Tree in Autumn. 


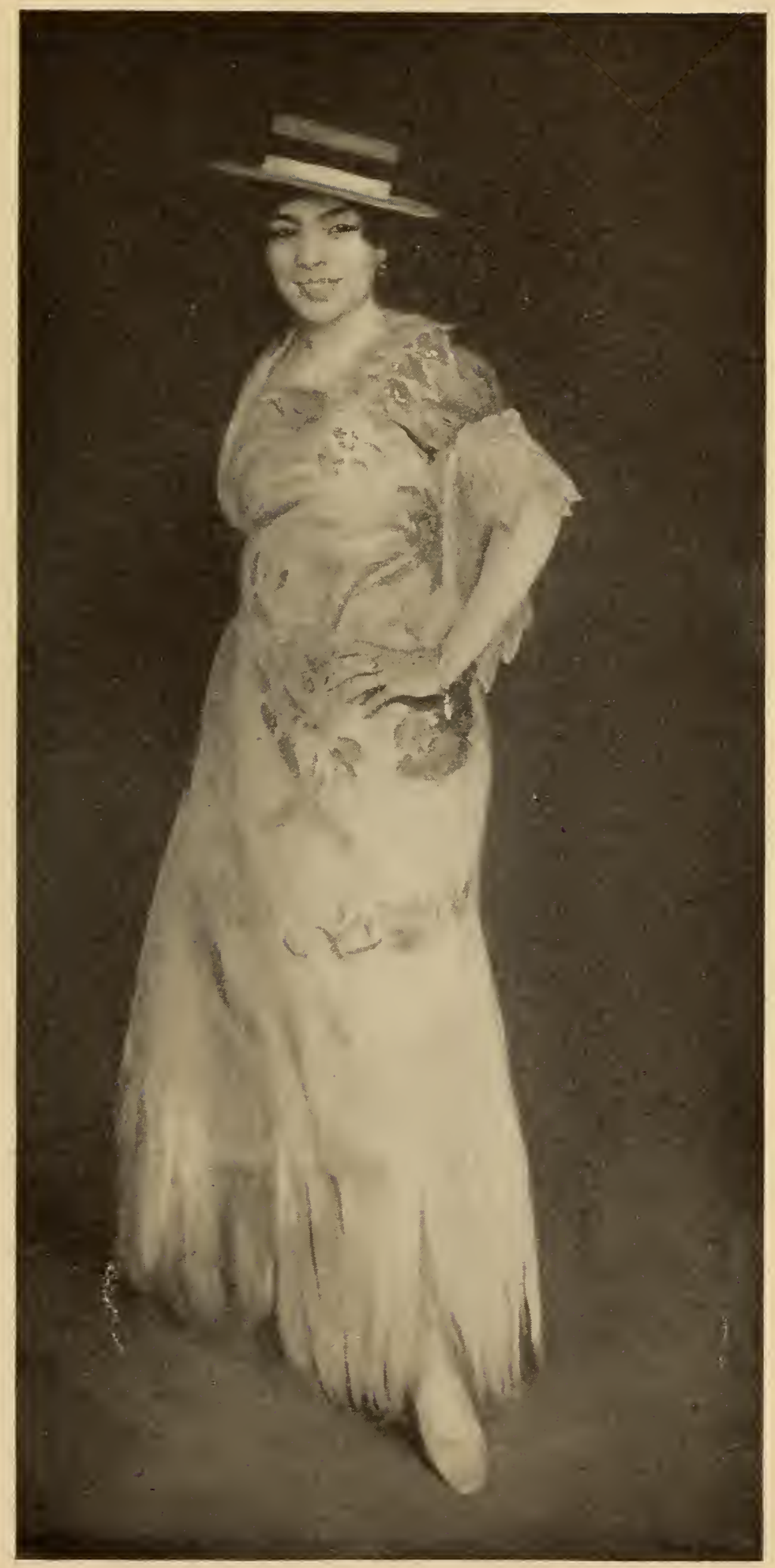

SPANISH DANCER. 

REID, ROBERT-Born Stockbridge, Mass., 1863. Pupil of Boston Museum; A. S. L., of N. Y.; Boulanger and Lefebvre in Paris. Medal, Columbian Exposition, Chicago, 1893; Clarke Prize, N. A. D., 1897; First Hallgarten Prize, N. A. D., 1898; Silver Medal for painting and Gold Medal for Mural Decoration, Paris Exposition, 1900; Silver Medal, Pan-American Expositon, Buffalo, 1901; Silver Medal, St. Louis, Exposition, 1904; Third Prize, Corcoran A. G., 1909; A. N. A., 1902 ; N. A., 1906 ; Member Nat. Inst. A. L.; Ten American Painters. Specialty, Mural Decoration. Also Teacher.

143. The Canna.

EATON, CHARLES WARREN-(See 108.)

144. November.

DIDIER-POUGET, W.-Born in Toulouse, 1864. Contemporary French painter.

\section{The Last Days.}

Loaned by Prinz Bros., Brooklyn, N. Y.

RANGER, HENRY W.-Born in New York State, 1858. Self taught. Bronze Medal, Paris Exposition, 1900; Silver Medal, PanAmerican Exposition, Buffalo, 1901; Gold Medal, Charleston Exposition, 1902 ; A. N. A., 1901; N. A., 1906; Member A. W. C. S.; Lotos Club. Specialty, Landscapes.

146. A Naonk Street.

Loaned by Wm. T. Evans, Montclair, N. J.

YATES, CULLEN-Born Bryan, O., Jan. 24, 1866. Pupil of $\mathrm{N}$. A. D. and Wm. M. Chase in New York; Ecole des Beaux Arts and Julian Academy under Laurens and Benjamin-Constant in Paris. Bronze Medal, St. Louis Exposition, 1904; Inness Prize, Salma. C., 1907. Member Salma. C., 1899; N. Y. W. C. C., 1904; A. W. C. S., 1905 ; A. N. A. Specialty, Landscapes.

147. The Approach.

HASSAM, CHILDE-(See 122.)

148. June.

EATON. CHARLES WARREN-(See 108.)

149. Evening in Connecticut.

HOWE, WILLIAM HENRY-Born Ravenna, Ohio, 1846. Pupil of Otto de Thoren and F. de Villefroy in Paris. Honorable Mention, New Orleans, 1885; Honorable Mention, Paris Salon, 1886; Third Class Medal, Paris Salon, 1888; Silver Medal, Paris Exposition, 1889; Temple Gold Medal, P. A. F. A., 1890; Medal, Columbian Exposition, Chicago, 1893; Grand Gold Medal, Crystal Palace, London, 1890; Gold Medal, Boston, 1890; Gold Medal, California, Mid-Winter Exposition, 1894; Gold Medal, Atlanta Exposition, 1895; Officer d'Academie, Paris, 1896; Chevalier of the Legion of Honor, 1899; Silver Medal, Pan-American Exposition, Buffalo, 1901; Member Jury of Awards, St. Louis Exposition, 1904; Member N. A., 1897; S. A. A., 1899; Salmagundi Club, 1891. Specialty, Landscape with Cattle.

150. Crossing the Ford. 
DEMING, EDWIN WILLARD-Born Ashland, O., Aug. 26, 1860. Pupil of Lefebvre and Boulanger in Paris. Silver Medal, A. A. S., 1892; Bronze Medal, St. Louis Exposition, 1904. Member N. Y. Arch. Lg., 1902; Mural P. Specialty, Mural Decoration.

151. The Spirit of Famine.

YATES, CULLEN-(S€e 147.)

152. Rye Field.

HENRI, ROBERT-(See 128.)

153. Spanish Dancer-El Tango.

DUSTIN, SILAS S.-Born West Richfield, O. Pupil of N. A. D. and of Wm. M. Chase. Member Salmagundi Club, 1902; A. Fund S. Specialty, Landscapes.

\section{Sleeping Village.}

CHURCH, FREDERICK STUART-Born Grand Rapids, Mich., 1842. Pupil of L. E. Wilmarth, Walter Shirlaw, N. A. D., and A. S. L., in New York. Silver Medal, St. Louis Exposition. Member N. A., 1885 ; S. A. A., 1890 ; A. W. C. S. ; N. Y. Etching C.

155. The Enchantress.

Loaned by Fred E. Sander, Seattle.

TER MEULEN, FRANCOIS PIETER-Born at The Hague, 1843. Pupil of J. Bakhuijsen. Silver Medal, Amsterdam, 1886. Gold Medals, Munich, 1891, 1892.

156. Coming from Pasture.

Loaned by R. C. \& N. M. Vose, Boston.

MORA, F. LUIS-(See 102.)

157. The Fortune Teller. 


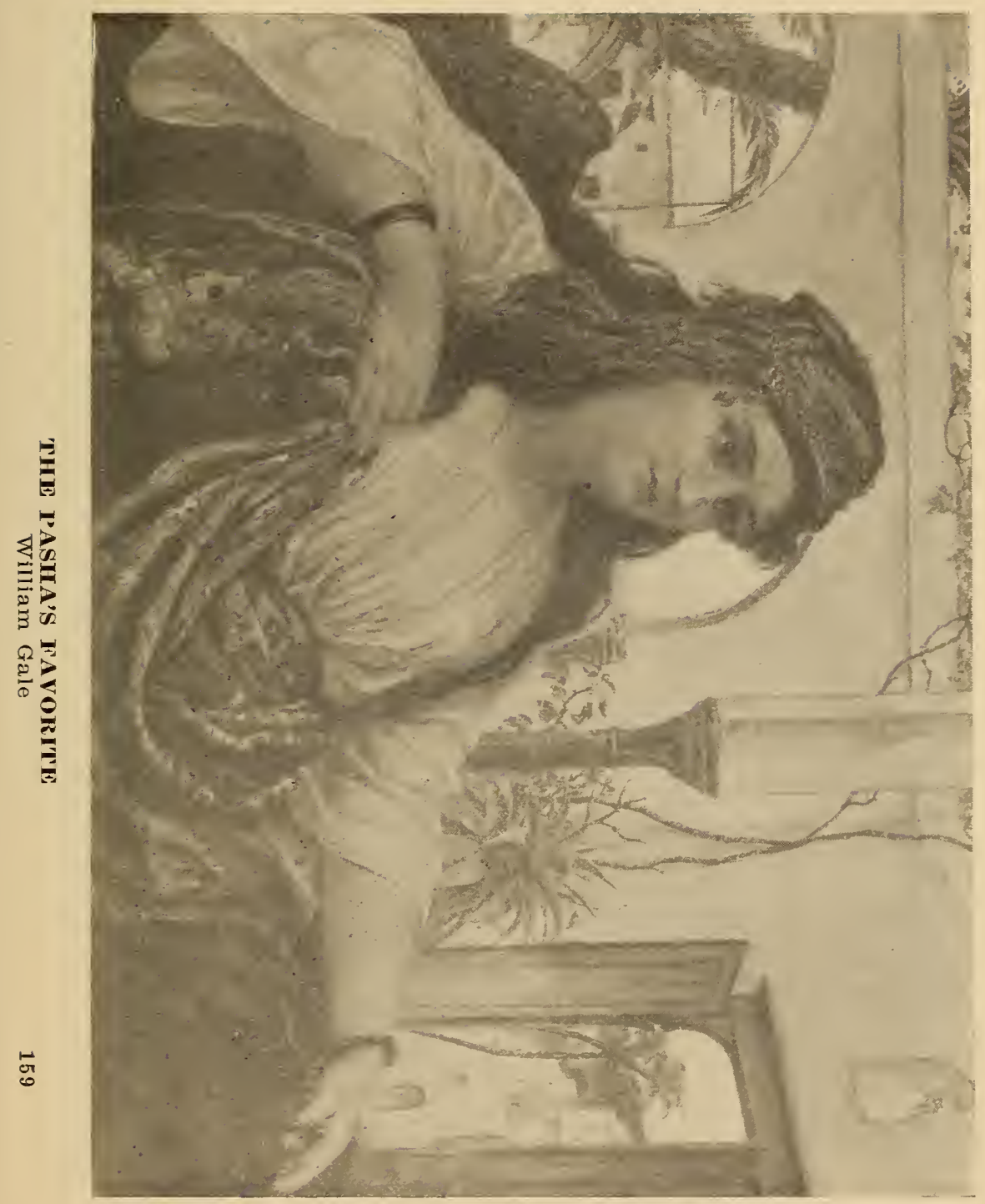





\title{
Gallery D
}

\author{
TWACHTMAN, JOHN H.-(See 106.)
}

158. Summer.

Loaned by Silas S. Dustin, New York.

GALE, WILLIAM--Contemporary English. Member of the Royal Academy.

159. Pasha's Favorite.

Loaned by Dr. Alfred Raymond, Seattle.

LATHROP, FRANCIS-Born at sea, near Hawaiian Islands, 1849. Pupil of Sir Edward Bourne-Jones in London. Gold and Silver Medals, Philadelphia, 1889; Bronze Medal, Pan-American Exposition, Buffalo, 1901; Member S. A. A., 1877; A. N. A., 1906; Mural Painters; New York Municipal A. S.; New York Arch. Lg., 1899; Century Association; Nat'l. Inst. A. L.

160. Madrelena.

MURPHY, JOHN FRANCIS-Born Oswego, N. Y., 1863. Second Hallgarten Prize, N. A. D., 1885; Webb Prize, S. A. A., 1887; Gold Medal Competition Prize, Fund Exhibition, New York, 1887; Medal, Columbian Exposition, Chicago, 1893; Evans Prize, A. W. C. S., 1894; Gold Medal, A. C. P., 1899; Honorable Mention, Paris Exposition, 1900; Silver Medal, Pan-American Exposition, 1901; Gold Medal, Charleston Exposition, 1902; Carnegie Prize, S. A. A., 1902; Silver Medal, St. Louis Exposition, 1904 ; A. N. A., 1885; N. A., 1887; Member S. A. A., 1901; A. W. C. S.; S. L. P.; Salmagundi Club, 1878; Brooklyn A. C., 1900 . Specialty, Landscape.

\section{Late September.}

SARGENT, JOHN SINGER-Born Florence, Italy, of American parents, 1856 Pupil of Carolus-Duran in Paris. Honorable Mention, Paris Salon, 1878; Second Class Medal, Paris Salon, 1881; Medal of Honor, Paris Exposition, 1889; Medal, A. C. P., 1890; Medal, Columbian Exposition, Chicago, 1893; Temple Gold Medal, P. A. F. A., 1894; Medal of Honor, Paris Exposition, 1900; Gold Medal, PanAmerican Exposition, Buffalo, 1901; Converse Gold Medal, P. A. F. A., 1903; Large Gold Medal, Berlin, 1903; Grand Prize, St. Louis Exposition, 1904; Grand Medal of Honor, Liege Exposition, 1905. Chevalier of the Legion of Honor, 1889. Member N. A., 1897; Copley S. (hon.); Paris S. A. P.; Soc. Nat. des Beaux Arts, Paris; Royal Academy, London; Century Assoc., New York; Nat. Inst. A. L. Specialty, Portraits.

\section{Venetian Water Carrier.}

Loaned by Frederick Crane, New York. 


\section{Midsummer Evening, Venice.}

INNESS, GEORGE-Born at Newburg; N. Y., 1825; died, 1894. Pupil of Regis Gignoux, 1868. In Italy, 1871-75. His pictures resemble the works of other artists in nothing. He was erratic, but possessed with a deep love and devotion to nature. In his later works he attained an excellence which placed him in the front rank of the best landscape painters of the world. Member of the National Academy of Design.

\section{Woods at "Milton.}

Loaned by $\mathrm{Wm}$. T. Evans, Montclair, N. J.

WILLIAMS, FREDERICK BALLARD-Born Brooklyn, N. Y., Oct. 21, 1872. Pupil of N. A. D. Bronze Medal, Pan-American Exposition, Buffalo, 1901; Silver Medal, A. A. S., 1902; Inness Prize, Salma. C., 1907. Member N. Y. W. C. C.; Salma. C., 1898; Lotos C.; A. N. A., 1907.

\section{Love and Sympathy.}

TANNER, HENRY OSSAWA-Born Pittsburg, Pa. Pupil of P. A. F. A. under Thomas Eakins; Laurens and Benjamin-Constant in Paris. Honorable Mention, Paris Salon, 1896; Third Class Medal, Paris Salon, 1897; Lippincott Prize, P. A. F. A., 1900; Silver Medal, Paris Exposition, 1900 ; Silver Medal, Pan-American Exposition, Buffalo, 1901; Silver Medal, St. Louis Exposition, 1904; Second Class Medal, Paris Salon, 1906; Harris Prize, A. I. C., 1906. Member Paris S. A. P. Specialty, Biblical Subjects.

166. Nicodemus.

DEWEY, CHARLES MELVILLE-Born Lowville, N. Y., 1851. Pupil of Carolus-Duran in Paris. Silver Medal, Pan-American Exposition, Buffalo, 1901; Silver Medal, St. Louis Exposition, 1904; A. N. A., 1903; N. A., 1907. Specialty, Landscapes.

\section{Sunset.}

DOUGHERTY, PAUL-Born Broolklyn, N. Y., 1877. Osborne Prize, $(\$ 500$,$) 1905. Member S. A. A., 1905$; A. N. A., 1906 ; N. A., 1907 ; Lotus C.; Salmagundi Club, 1903; A. W. C. S.

\section{Northern Sky.}

REDFIELD, EDWARD W.-Born Bridgeville, Del., 1868. Pupil of P. A. F. A.; Bouguereau and Robert-Fleury in Paris. Gold Medal, A. C. P., 1896; Bronze Medal, Paris Exposition, 1900; Bronze Medal, Pan-American Exposition, Buffalo, 1901; Temple Gold Medal, P. A. F. A., 1903; Second Hallgarten Prize, N. A. D., 1904; Shaw Fund Prize, S. A. A., 1904; Silver Medal, St. Louis Exposition, 1904; Jennie Sesnan Gold Medal, P. A. F. A., 1905; Medal of Second Class, C. I., Pittsburg, 1905; Webb Prize, S. A. A., 1906; Gold Medal of Honor, P. A. F. A., 1907; Third Prize, Corcoran A. G., 1907; First Clarke Prize, and Corcoran Gold Medal, Corcoran A. G., 1908. Member S. A. A., 1903; A. N. A., 1904; N. A., 1906; A. C. P.; Fellowship P. A. F. A. Specialty, Landscapes.

169. Harbor of Boulogne. 


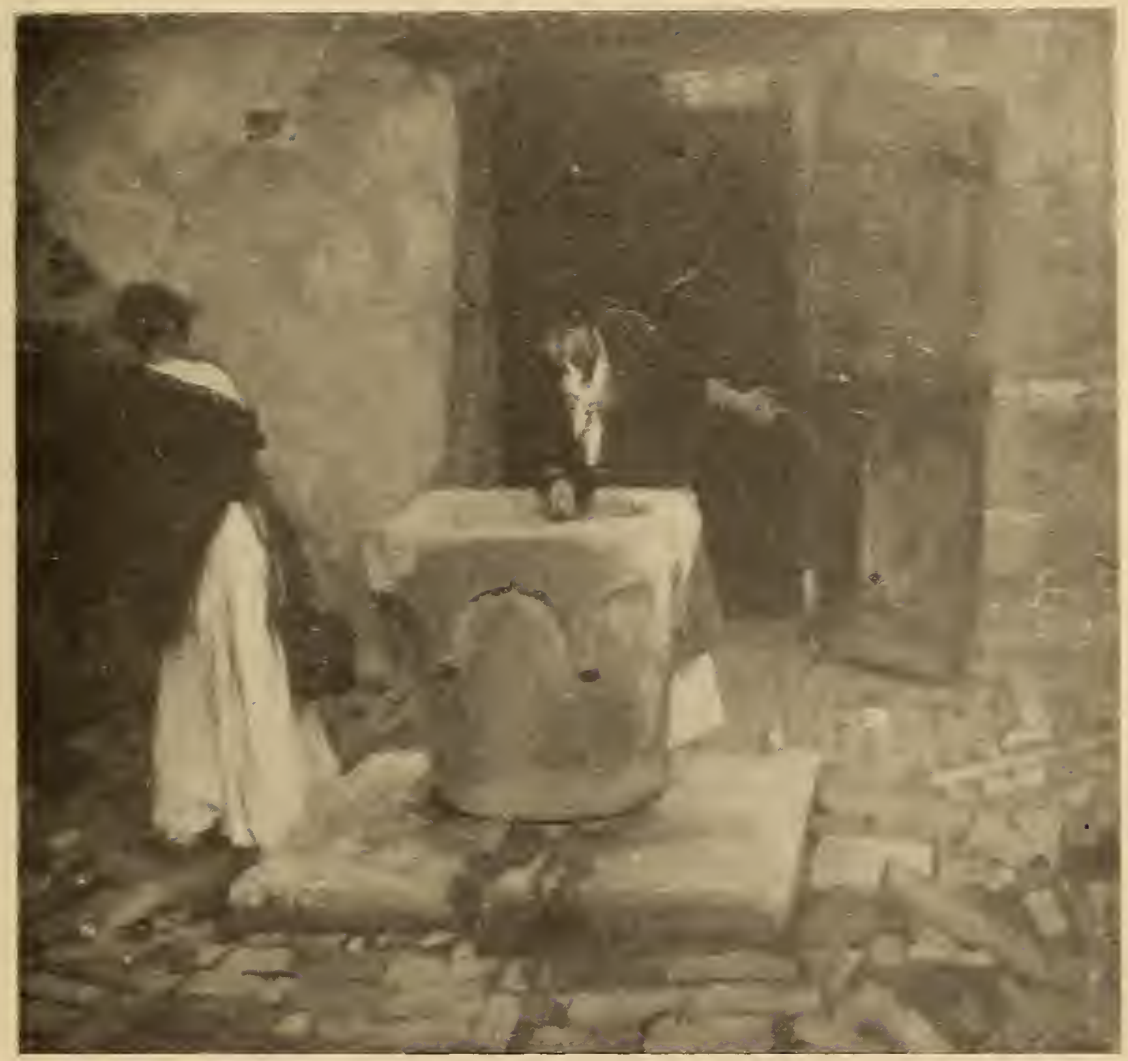

VENETIAN WATER CARRIER

John S. Sargent 

SMILLIE, GEORGE HENRY-Born New York, Dec. 29, 1840. Pupil of James M. Hart. First Prize, American Art Assoc., New York, 1885; Bronze Medal, St. Louis Exposition, 1904. Member N. A. 1882 ; A. W. C. S.; Century Assoc.; Lotos C. Specialty, Landscapes.

\section{A Group of Cedars.}

COOPER, EMMA LAMPERT-Born Nunda, N. Y. Pupil of Harry Thompson in Paris; J. Kever in Holland; Wm. M. Chase in New York. Medal Columbian Exposition, Chicago, 1893; Atlanta Exposition, 1895; Gold Medal, A. A. S., 1902; Bronze Medal, St. Louis Exposition, 1904; Prize, N. Y. Woman's A. C., 1907. Member Plastic Club; Phila. W. C. C.; Fellowship P. A. F. A.; N. Y. W. C. C.; N. Y. Woman's A. C.; W. A. A. of Canada; Women's International A. C., London.

\section{Canal at Lisieux, France.}

DE HAVEN, FRANK-Born Bluffton, Ind., 1856. Pupil of George H. Smillie. Inness Prize, Salmagundi Club, 1900; Shaw Prize, Salmagundi Club, 1901; Honorable Memtion Pan-American Exposition, Buffalo, 1901; Silver Medal, Charleston Exposition, 1902; Silver Medal, St. Louis Exposition, 1904. A. N. A., 1902; Member Salmagundi Club, 1899. Specialty Landscapes.

172. Night Fall.

LIE, JONAS-(See 141.)

173. After the Snow Fall.

BLAKELOCK, RALPH ALBERT-Born New York, 1847. Self taught. Honorable Mention, Paris Exposition, 1900. Specialty, Landscapes.

174. Sunset at Sea.

Loaned by Wm. T. Evans, Montclair, N. J.

RYDER, CHAUNCEY F.-(See 127.)

175. That Which the Sea Gives Up.

VAN LAER, ALEXANDER T.-(See 112.)

176. October Day, Connecticut.

DAVIES, ARTHUR B.-(See 130.)

177. Silver Springs.

Loaned by William Macbeth, New York.

SMEDLEY, WILLIAM T.-Born Chester Co., Pa., 1858. Pupil of P. A. F. A.; Laurens in Paris. Evans Prize, A. W. C. S. 1890; Bronze Medal, Paris Exposition, 1900; Bronze Medal for Painting and Silver for Drawing, Pan-American Exposition, Buffalo, 1901; Proctor Prize, N. A. D., 1906. Member N. A., 1905; S. A. A., 1882; A. W. C. S.; A. Aid S.; S. I.; Century Assoc.

178. Booklovers. 
GRUPPE, CHARLES P.-Born Pictou, Canada, 1860. Studied in Holland; chiefly self-taught. Gold Medal, Rouen; Gold Medal, A. A. S., 1902; Two Gold Medals, Paris; Silver Medals, for oils and for water color, St. Louis Exposition, 1904. Member Pulchre Studio, The Hague; Arti. Amsterdam; N. Y. W. C. C.; Salmagundi Club, 1893; Rochester A. C.

179. Pasture Along the Dyke.

SISLEY, ALFRED-French; 1840-1899. Studied for two years with Gleyre, after which he recognized Nature as his only master.

180. Paysage pres Moret.

Loaned by Durand-Ruel \& Sons, New York.

WEEKS, EDWIN LORD-Born in Boston, 1849; died, 1903. Pupil of Bonnat, Ecole des Beaux Arts, Paris. Medal of Salon, 1889; E. U. (gold), 1889. Chevalier Legion d'Honneur, 1896.

181. Court of the Palace of the Seths, Agineer, India.

Loaned by R. C. \& N. M. Vose, Boston.

REDFIELD, EDWARD W.-(See 169.)

182. Morning, Boulogne.

FOSTER, BEN-(See 136.)

183. Now the Day is Over.

GENTH, LILLIAN M.-(See 110.)

184. The Pool.

SMITH, W. GRANVILLE-Born Granville, N. Y., 1870. Pupil of Walter Satterlee and A. S. L.; studied in Europe. Third Hallgarten Prize, N. A. D., 1900; Bronze Medal, Charleston Exposition, 1902; Evans Prize, A. W. C. S., 1905; First Prize, Worcester, 1906; Honorable Mention, C. I., Pittsburg, 1907; Inness Gold Medal, N. A. D., 1908. Member A. W. C. S.; Salma. C., 1898; S. I.

185. The Old Mill.

DEWEY, CHARLES MELVILLE-(See 167.)

186. Homeward.

187. Sunshine and Shadow.

ROBINSON, WILLIAM S.-Honorable Mention, Paris Exposition, 1900; Honorable Mention, Pan-American Exposition, Buffalo, 1901; Gold Medal, A A S., 1902; Bronze Medal, St. Louis Exposition, 1904; A. N. A., 1901 ; Member A. W. C .S.; N. Y. W. C. C.; B. A. C.; Salma. C., 1897 ; Lotos C.

188. Passing Shower. 


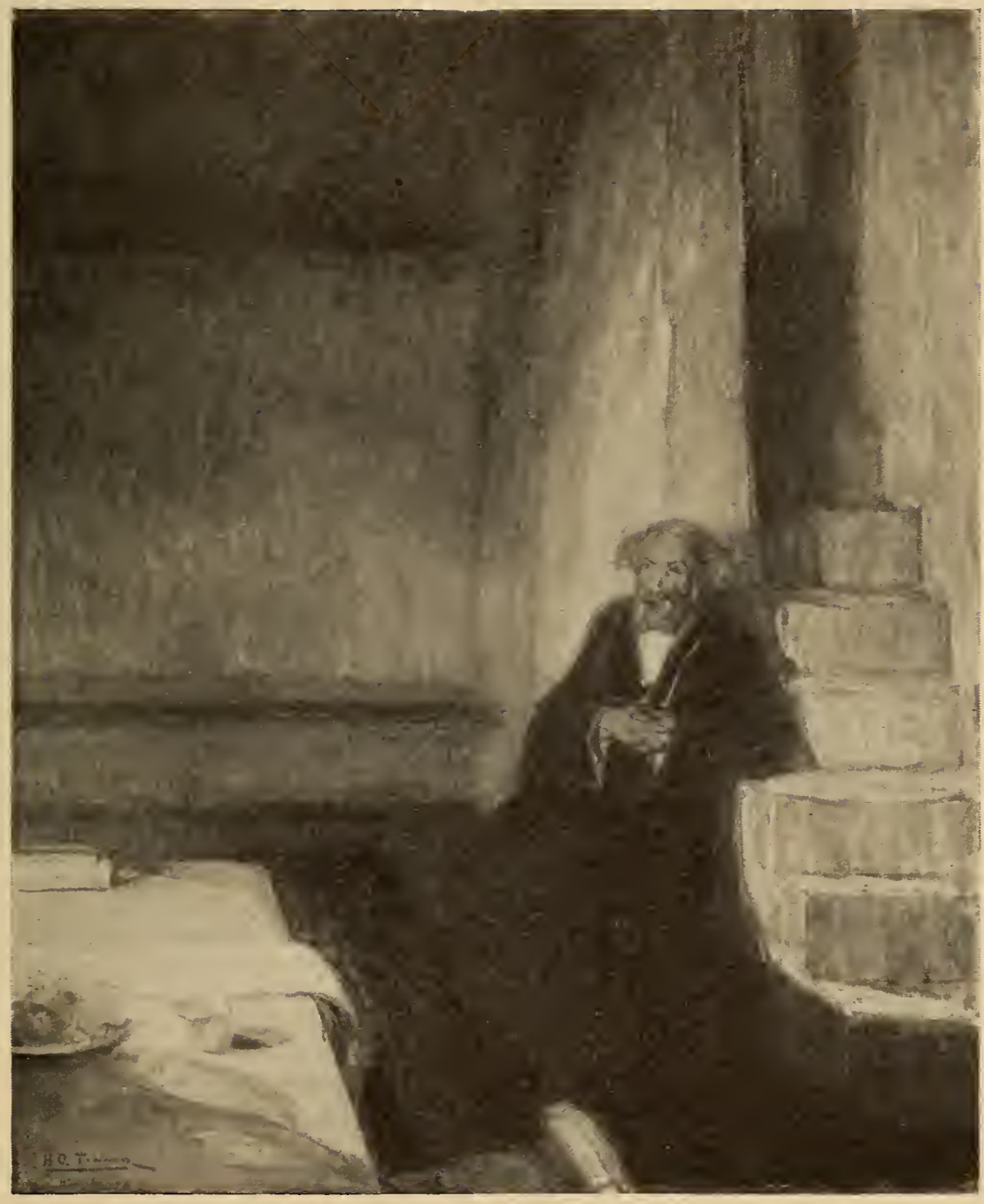

NICODEMUS.

Henry O. Tanner. 

CARLSEN, EMIL-Born Copenhagen, Denmark, 1853. Came to United States in 1872. Pupil of Danish Royal Academy as Architect. Second Inness Prize, Salma. C., 1904; Shaw Purchase, S. A. A., 1904; Gold Medal, St. Louis Exposition, 1904; Webb Prize, S. A. A., 1905; Inness Medal, N. A. D., 1907. Member S. A. A., 1902; A. N. A., 1904; N. A., 1906; Salma. C., 1903.

189. The Wind of the East.

Loaned by Mrs. Kate Linde, Hoboken, N. J.

DEWEY, CHARLES MELVILLE-(See 167.)

190. Druid Oak.

INNESS, GEORGE, JR.-Born in Paris, 1854. Pupil of his father, George Innes. Gold Medal, Paris Salon, 1899; Silver Medal, Pan-American Exposition, 1901; Silver Medal, Charleston Exposition, 1902; Gold Medal, A. A. S., 1902. Member N. A., 1899; S. A. A., 1880 ; B. A. C.; Salmagundi Club, 1876, (life, 1899); Black and White (Pres.); A. Fund S.; French Academy, 1902.

191. Landscape and Cattle.

Loaned by Wm. S. Hurley, Brooklyn, N. Y.

SMITH, W. GRANVILLE-(See 185.)

192. Moonlight Shadows.

DEWEY, CHARLES MELVILLE-(See 167.)

193. Evening, Marshes of Essex, Mass.

BECKWITH, J. CARROLL-(See 124.)

194. Waldweben.

WIEGAND, GUSTAVE-Born Bremen, Germany, 1870. Pupil of Wm. M. Chase; Dresden Royal Academy under Eugene Bracht. Bronze Medals, St. Louis Exposition, 1904; Second Hallgarten Prize, N. A. D., 1905.

195. The Belated Shepherd.

REID, ROBERT-(See 143.)

196. Woman Bathing.

Loaned by Francis Lathrop, New York.

DEARTH, HENRY GOLDEN-Born Bristol, R. I., 1863. Pupil of Ecole des Beaux Arts and Aime Morot in Paris; Webb Prize, S. A. A., 1893; Bronze Medal, Paris Exposition, 1900; Silver Medal, Pan-American Exposition, Buffalo, 1901; Silver Medal, Charleston Exposition, 1902; A. N. A., 1902; N. A., 1906; Member S. A. A., 1894; Century Assoc.

197. The Day's Work Over. 
INNESS, GEORGE-(See 164.)

198. Return to the Farm.

Loaned by Wm. S. Hurley, Brooklyn, N. Y.

MURPHY, J. FRANCIS-(See 161.)

199. Marshy Meadows.

Loaned by William Macbeth, New York.

STEVENSON, ROBERT MACAULEY-Born in Glasgow, 1854. Glasgow school. Represented in the National Gallery of Belgium; National Gallery of Germany; National Gallery of Bavaria; National Gallery of Bohemia, Prague; Gallery of Barcelona; Gallery of Weimar; Museum of Fine Arts, St. Louis. Corresponding Member of the Secession, Munich. Gold Medal, International Art Exhibition, Munich, 1893; Silver Medal, International Exhibition, Brussels, 1897; Diploma of Honor, International Exhibition, Barcelona, 1894.

2.00. The Home Meadow.

Loaned by The Albright Art Gallery, Buffalo.

SARGENT, JOHN SINGER-(See 162.)

201. Mrs. Fiske Warren and Daughter.

Loaned by Mrs. Fiske Warren, Boston.

WEIR, J. ALDEN-(See 129.)

202. June.

OCHTMAN, LEONARD-Born Zonnemaire, Holland, 1854. Self taught. Prize Brooklyn A. C., 1891; Medal, Columbian Exposition, 1893; Gold Medal, A. C. P., 1894; Silver Medal, Pan-American Exposition, Buffalo, 1901; Silver Medal, Charleston Exposition, 1902; Morgan Prize, for W. C.; Salma C., 1902; Shaw Fund Prize; S. A. A., 1902; Inness Gold Medal, N. A. D., 1903; Evans Prize, Salmagundi Club, 1903, Woodward Purchase Fund, Brooklyn Inst. A. S., 1903; Webb Prize, S. A. A., 1904; Two Gold Medals, (oil and water color) St. Louis Exposition, 1904; Second Corcoran Prize, Washington S. A., 1905; Rhead Purchase Fund, Richmond Art C., 1905; Inness Prize, Salmagundi Club, 1906; Evans Prize, Salmagundi Club, 1907; A. N. A., 1898; N. A., 1904; Member S. A. A., 1895; A. W. C. S.; N. Y. W. C. C.; S. Landscape P.; Brooklyn A. C.; A. Fund S.; A. Aid S.; Salmagundi C., 1901; Lotus Club. Specialty, Landscapes.

203. In April.

WILES, IRVING RAMSAY-Born Utica, N. Y., 1861. Pupil of of his father, L. M. Wiles; William M. Chase and Carroll Beckwith in New York; Carolus-Duran in Paris. Third Hallgarten Prize, N. A. D., 1886; Clarke Prize, N. A. D., 1889; Honorable Mention, Paris Exposition, 1889; Medal Columbian Exposition, Chicago, 1893; Evans Prize, A. W. C. S., 1897; Medal, Tennessee Centennial, Nashville, 1897; Shaw Prize, S. A. A., 1900; Bronze Medal, Paris Exposition, 1900; Gold Medal, Pan-American Exposition, Buffalo, 1901; First Corcoran Prize, S. Washington A., 1901; Gold Medal, St. Louis Exposition, 1904. Member N. A., 1897; S. A. A., 1887; A. W. C. S.; N.

X. W. C. C.; Century Assoc.

204. Mrs. Gilbert. 


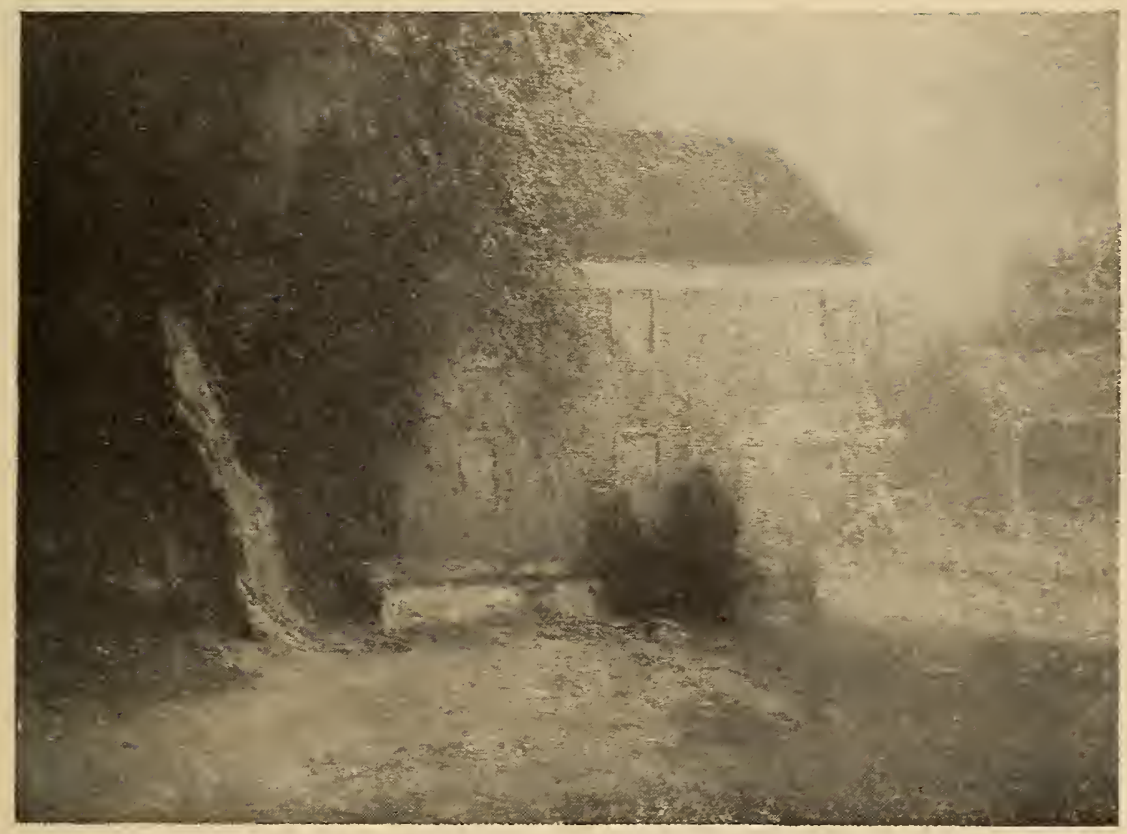

THE OLD MILL

WV. Granville Smith 



\section{Gallery E}

MILLET, FRANCIS DAVIS-Born Mattapoisett, Mass., 1846. Pupil of the Royal Academy of Arts in Antwerp, under Van Lerius and De Keyser. Silver and Gold Medals of Honor in 1872-1873 at Royal Academy, Antwerp; Silver Medal, Paris Exposition, 1889; Medal, Columbian Exposition, Chicago, 1883; Pan-American Exposition, 1901; Legion of Honor. Was special correspondent for the "Daily News" during the Russo-Turkish war, 1877; for this work he received the Roumanian Iron Cross and the Order of Chevalier St. Anne and of St. Stanislaus from the Russian government. Special correspondent of the "London Times" at Manila during the Spanish war. Director of Decorations, Columbian Exposition, 1893. Member N. A., 1885 ; S. A. A., 1880 ; A. W. C. S.; IIural P.; B. A. C.; A. Fund S.; Inst. Painters in Oil Colors; Century Association; S. I.

205. The Black Sheep.

POTTHAST, EDWARD H.-(See 126.)

206. Late Autumn.

HASSAM, CHILDE-(See 122.)

207. Aphrodite.

CLAYS, PAUL JEAN-Born at Bruges, 1812; died at Brussels, 1901. Pupil in Paris of Gudin. Leading Marine Painter of Modern Belgium.

208. Moonrise on the Scheldt.

Loaned by R. C. \& N. M. Vose, Boston.

PALMER, WALTER LAUNT-Born Albany, N. Y., 1854. Pupil of F. E. Church, in Hudson, N. Y.; Carolus-Duran, in Paris. Second Hallgarten Prize, N. A. D., 1857; Medal, Columbian Exposition, Chjcago, 1893; Gold Medal, A. C. P., 1894; Evan's Prize, A. W. C. S., 1895; First Prize, B. A. C., 1895; Second Prize, Centennial Exposition, Nashville, Tenn., 1897; Honorable Mention, Paris Exposition, 1900 ; Silver Medal for Water Color, Pan-American Exposition, Buffalo, 1901; Silver Medal for W. C., Charleston Exposition, 1902; Bronze Medal for oil and Silver Medal for water color, St. Louis Exposition, 1904 ; Silver Medal, Philadelphia, 1907; Member N. A., 1897; S. A. A., 1881 ; N. Y. W. C. C., 1881 ; A. W. C. S.; Salmagundi Club, 1901; Century Association. Specialty, Winter Landscape.

209. The Pine Grove. 
MINOR, ROBERT C.-Born New York, 1840; died, 1904. Pupil of Diaz. He was a National Academician, and a member of the American Water-Color Society.

210. Eventide.

Loaned by Wm. T. Evans, Montclair, N. J.

MARIS, JACOB-Born at The Hague, August 25, 1838; died, 1899. Pupil of Van Hove Strobel and of the Antwerp Academy. Medals at Amsterdam, Paris, and Munich. Knight of the Orders of the Dutch Lion and St. Michael of Baveria.

211. The Tow.

Loaned by R. C. \& N. M. Vose, Boston.

MOORE, ERNEST-Contemporary English portrait painter.

212. Mrs. Ernest Moore.

RYDER, CHAUNCEY F.-(See 127.)

213. The Dunes.

Loaned by William Trevor, New York.

MORET, HENRY-Born Cherbourg, France, 1856.

214. Temps, gris-lle de Groix.

Loaned by Durand-Ruel \& Sons, New York.

PAXTON, WILLIAM M.-Born Baltimore, Md., 1869. Pupil of Ecole des Beaux Arts, under Gerome in Paris; Denis M. Bunker in New York. Honorable Mention, Pan-American Exposition, Buffalo, 1901; Bronze Medal, St. Louis Exposition, 1904. Member Copley S., 1894 .

215. Glow of Gold and Gleam of Pearl.

RITSCHEL, WILLIAM-Born Nurnberg, Bavaria, Germany, 1864. Pupil of Prof. Linder, A Kaulbach in Munich. Member Salma. C., 1901; Kunstverein, Munich. Specialty, Marines.

216. Gathering Kelp, Maine.

WEIR, J. ALDEN-(See 129.)

217. Pelham's Lane.

DESSAR, LOUIS PAUL-(See 111.)

218. Moonlight.

Loaned by The Folsom Co., New York. 


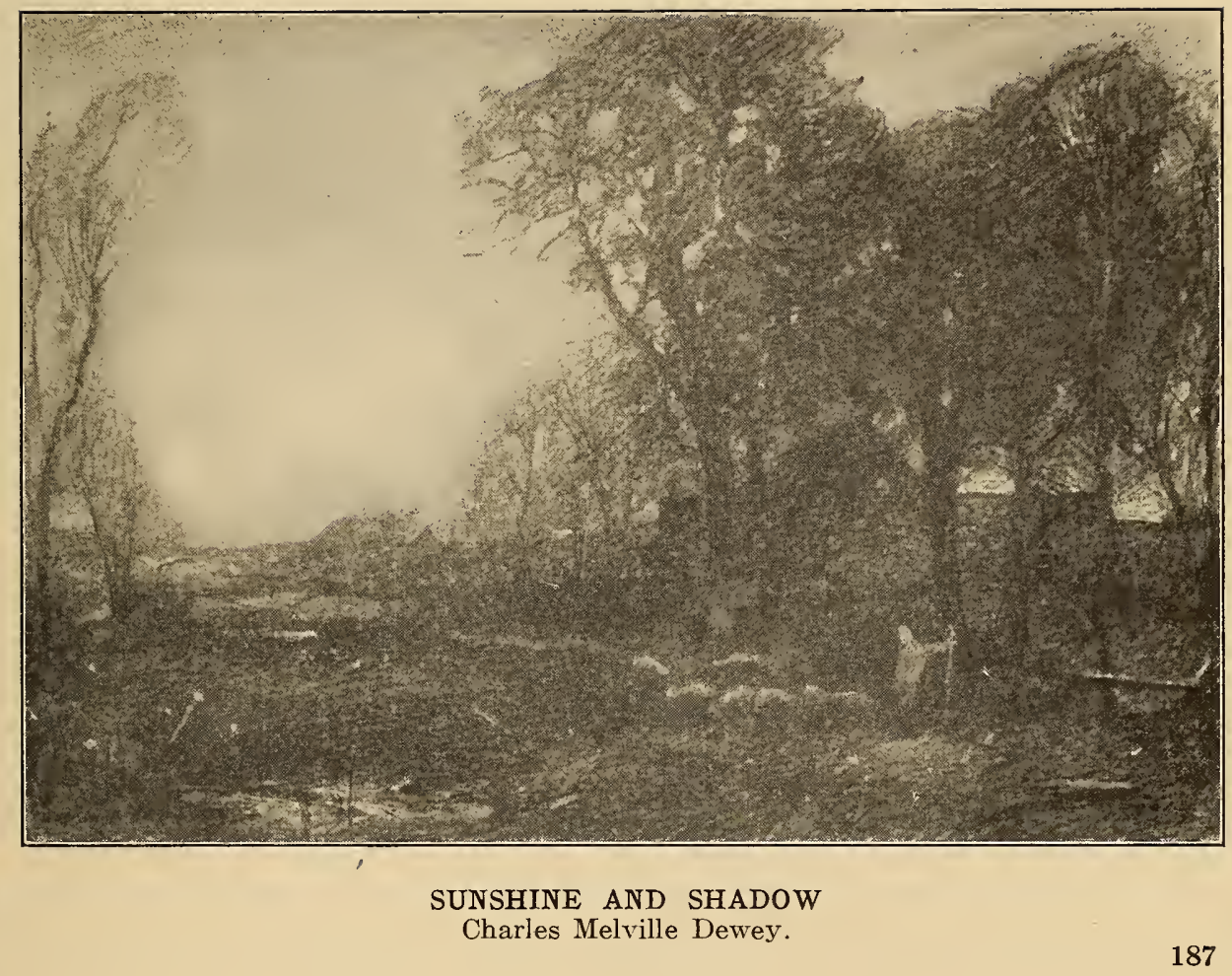



MURPHY, HERMAN DUDLEY-Born Marlboro, Mass., 1867. Pupil of Laurens in Paris. Bronze Medal, Pan-American Exposition, Buffalo, 1901; Silver Medal, St. Louis Exposition, 1904. Member Copley S., 1886; N. Y. W. C. C.; St. Botolph C.; Salmagundi Club; Boston W. C. C. Specialty, Portraits and Carved Frames. Also Teacher.

219. The Edge of the Hill.

CASSATT, MARY-Born Pittsburg, Pa. Pupil of Manet in Paris. Chevalier of Legion of Honor of France, 1904. Lippincott Prize, P. A. F. A., 1904.

220. Apres le bain.

Loaned by Durand-Ruel \& Sons, New York

BLAKELOCK, RALPH A.-(See 174.)

221. Seal Rocks, California.

Loaned by Wm. S. Hurley, Brooklyn, N. Y.

RITSCHEL, WILLIAM-(See 216.)

222. Traveling Shadows.

D'ESPAGNAT, GEORGES-Born Melun, France, 1870.

223. Riviere de Cagnes.

Loaned by Durand-Ruel \& Sons, New York.

GEROME, JEAN-LEON-French; 1824-1904. Pupil of Paul Delaroche. Medals of Salon, 1847, 1848, 1874 (d'Honneur); E. U., 1855, 1867 (d'Honneur.) NIembre de I'Institut, 1865. Chev. Legion d'Honneur, 1855; Officier, 1867; Commander, 1878.

224. The Grief of the Pasha.

Loaned by the Art Institute of Chicago.

MOORE, HRNEST-(See 212.)

225. Governor J. H. McGraw.

Loaned by John H. McGraw, Seattle.

BEAL, GIFFORD-Third prize $(\$ 100.00)$, Worcester, Mass., 1903. Bronze Medal, St. Louis Exposition, 1904. A. N. A. (elect).

226. Lands End, Cornwall.

HENRI, ROBERT-(See 128.)

227. El Picador. 
REDFIELD, EDWARD W.--(See 169.)

228. The Quarry.

JONES, HUGH BOLTON-Born Baltimore, Md., 1848. Studied in France. Bronze Medal, Paris Exposition, 1889; Medal, Columbian Exposition, Chicago, 1893; Bronze Medal, Paris Exposition, 1900: Webb Prize, S. A. A., 1902; Shaw Fund Prize, S. A. A., 1902; Gold Medal, St. Louis Exposition, 1904. Member N. A., 1883; S. A. A., 1881; A. W. C. S.; N. A. C.; A. Fund S.; Century Association. Specialty, Landscapes.

229. Cloud Shadows.

MOORE, ERNEST-(See 212.)

230. Dr. Allerton Cushman.

HENRI, ROBERT-(See 128.)

231. Marine, Maine Coast.

BRECKENRIDGE, HUGH H.-Born Leesburgh, Va., 1870. Pupil of P. A. F. A.; Bouguereau, Doucet and Ferrier in Paris. First Toppan Prize and European Scholarship, Schools of P. A. F. A.; Medal, Atlanta Exposition, 1895; Honorable Mention, Paris Exposition, 1900; Medal, Pan-American Exposition, Buffalo, 1901; Second Corcoran Prize, S. Washington A., 1903; Gold Medal, A. C. P.. 1907. Member International Jury of Awards, St. Louis Exposition, 1904. Member N. Y. W. C. C.; Phila. W. C. C.; Fellowship P. A. F. A. Secretary of the Faculty and Instructor in P. A. F. A. since 1894.

232. Phlox.

DU MOND, FRANK VINCENT-Born Rochester, N. Y., 1865. Pupil of Boulanger, Lefebvre and Benjamin-Constant in Paris. Gold Medal, Atlanta Exposition, 1895; Gold Medal, Boston; Silver Medal for paintings and illustrations, Pan-American Exposition, Buffalo, 1901; Silver Medal, St. Louis Exposition, 1904; A. N. A., 1900; N. A., 1906; Member S. A. A., 1905; A. S. L., of N. Y.; N. Y. Arch. Lg.; Mural P.; S. I.; Lotus Club; Salmagundi Club, 1900; Director of Dept. of Fine Arts, Lewis and Clark Exposition, Portland, Ore., 1905. Instructor at A. S. L. of N. Y.

\section{The South Wind.}

SLOAN, JOHN-Born Lock Haven, Pa., Aug. 2, 1871. Pupil of P. A. F. A. and of Robert Henri in Philadelphia. Member Eight American Painters; S. I.

234. The Coffee Line.

DAVIS, CHARLES H.-(See 142.)

235. A Fitful Gleam.

Loaned by William Macbeth, New York. 


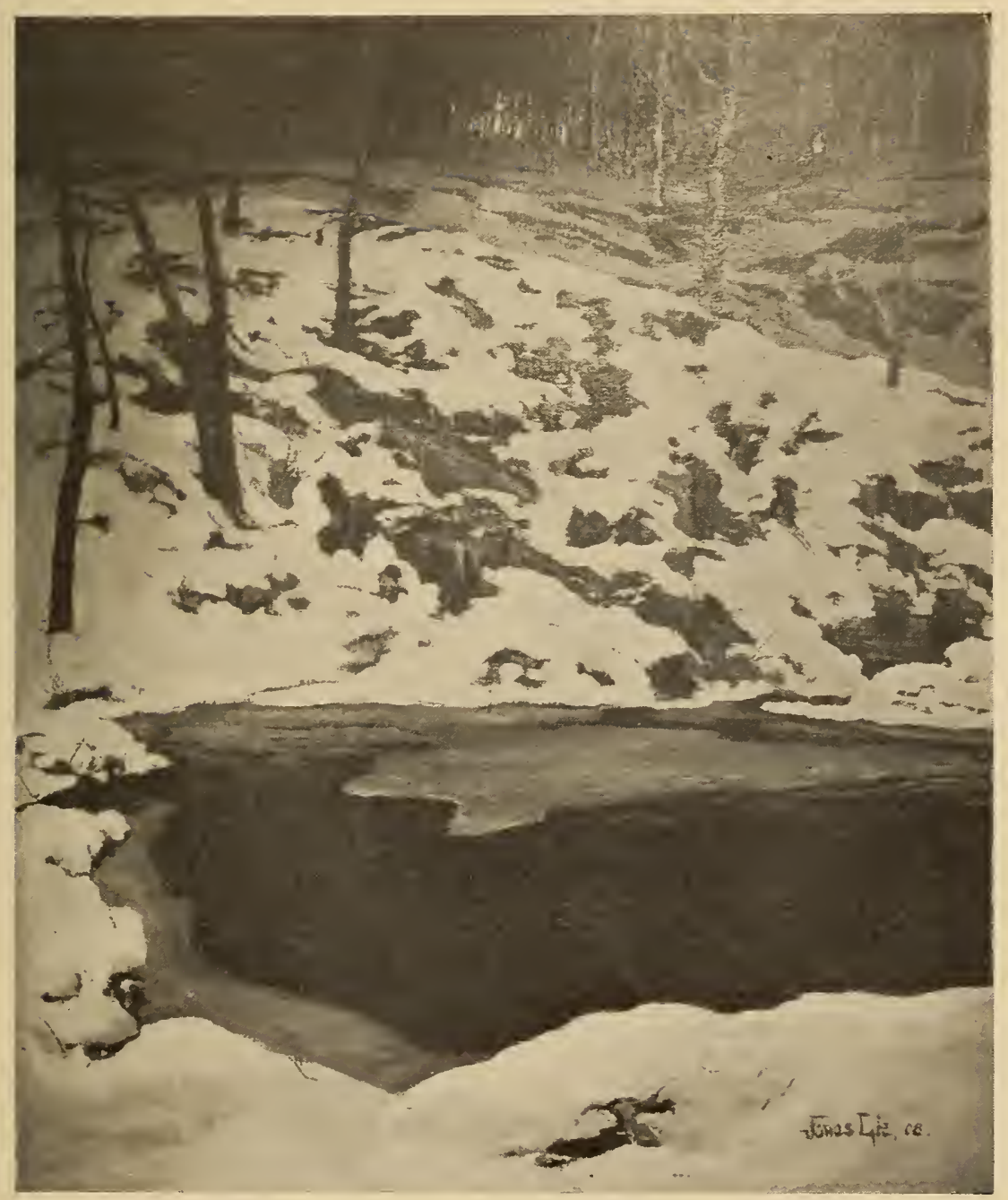

THE HEART OF THE WOODS

Jonas Lie 

SHIRLAW, WALTER-Born Paisley, Scotland, Aug. 6, 1838. Pupil of Munich Royal Academy and of Raab, Wagner, Ramberg, and Lindenschmidt in Munich. Medal, Royal Academy, Munich; Medal, Centennnial Exposition, Philadelphia, 1876; Honorable Mention, Paris Exposition, 1889; Lotos Club Fund Purchase, N. A. D., 1895; Silver Medal, Pan-American Exposition, Buffalo, 1901; Silver Medal, St. Louis Exposition, 1904. One of the founders of the S. A. $A$., and its president for first and second terms. Member N. A., 1888; S. A. A.; Century Assoc.; A. W. C. S.; Mural P.; A. Aid S.

\section{Flying Geese.}

Loaned by Wm. S. Hurley, Brooklyn, N. Y.

ALEXANDER, JOHN W.-Born Allegheny, Pa., Oct. 7, 1856. Studied in Munich, Venice and Florence and with Frank Duvenick. Temple Gold Medal, P. A. F. A., 1897; Lippincott Prize, P. A. F. A., 1899; Gold Medal Paris Exposition, 1900; Gold Medal, Pan-American Exposition, Buffalo, 1901; First Corcoran Prize, S. Washington A. 1903; Gold Medal, St. Louis Exposition, 1904; Chevalier Legion of Honor, 1901. Member Society Beaux-Arts; Munich Secessionists; N. A., 1902; S. A. A., 1901; N. Y. Arch Lg., 1901; Mural P.; Century Assoc.; International Society, London; Vienna Secessionists (hon.); Nat'l Inst A. L.

237. The Butterfly.

WILLIAMS, FREDERICK BALLARD-(See 165.)

238. The Showery Valley.

RICO, MARTIN-Born in Madrid; died, 1908. Pupil of Frederico de Madrazo; studied in Paris and Rome.

239. Venice.

Loaned by Geo. A. Dowden, Newark, N. J.

OCHTMAN, LEONARD-(See 203.)

240. Dawn.

PALMER, WALTER L.-(See 209.)

241. The Burdened Branch.

COOPER, COLIN CAMPBELL_-Born Philadelphia, Pa. Pupil of P. A. F. A.; Julian Academy and other schools in Paris. Bronze Medal, Atlanta Exposition, 1895; Gold Medal, A. A. S., 1902; Wm. T. Evans Prize, A. W. C. S., 1903; Sesnan Prize, P. A. F. A., 1904 ; Member International Jury of Awards, St. Louis Exposition, '04; Gold Medal, A. C. P., 1905; Member N. Y. W. C. C.; Fellowship P. A. F. A.; Salmagundi Club, 1904; American W. C. Soc.; Specialty Street Scenes.

242. Grand Central Station.

DE BOCK, THEOPHILE-Born in Harlem, 1851; died, 1904.

243. The Pool.

Loaned by R. C. \& N. M. Vose, Boston. 
ANSCHUTZ, THOMAS P.-Born Newport, Ky., Oct. 5, 1851. F'upil of N. A. D.; P. A. F. A.; Doucet and Bouguereau in Paris. Fonorable mention, A. C. P., 1901; Silver Medal, St. Louis Exposition, 1904. Member Fellowship P. A. F. A.; Phila. W. C. C.; Faculty of P. A. F. A. Summer School at Darby, Pa.

244. Girl in Green Dress.

PISSARRO, CAMILLE-Born in St. Thomas, Antilles; 1830-1903. Pupil of A. Melbye and Corot.

\section{Soleil couchant-temps gris-Rouen.}

Loaned by Durand-Ruel \& Sons, New York.

GAY, EDWARD-Born Dublin, Ireland, 1837. Pupil of J. M. Hart in New York; J. Schirmer and Lessing in Karlsruhe, Germany. Prize of $\$ 2,000$ in Competitive Exhibition, American Art Association for the landscape "Broad Acres," presented to the Metropolitan Museum, 1887; Medal, Midwinter Exhibition, San Francisco; Medal, New Orleans Exposition, 1885; Bronze Medal, PanAmerican Exposition, Buffalo, 1901; Shaw Purchase, S. A. A. 1903; Bronze Medal, St. Louis Exposition, 1904; George Inness Gold Medal, N. A. D., 1905; N. A., 1907; Member N. Y. W. C. C.; A. Fund S.

\section{My Lady's Estate.}

COUSE, E. IRVING-(See 121.)

\section{The Connoisseur.}

NETTLETON, WALTER-Born New Haven, Conn., 1861. Pupil of Boulanger and Lefebvre in Paris. Honorable mention, Paris Salon, 1892; Silver Medal, St. Louis Exposition, 1904. Member S. A. A., 1901; A. N. A., 1905; Also Teacher. Specialty, Landscapes.

\section{Early April in New England.}

DAVIS, CHARLES H.-(See 142.)

\section{Clouds and Hills.}

KOOPMAN, AUGUSTUS-Born Charlotte, N. C., 1869. Pupil of P. A. F. A.; Ecole des Beaux Arts, Bouguerean and Robert-Fleury in Paris. Second Wanamaker Prize, Paris, A. A. A., 1898; First Clarke Prize, Paris, A. A. A., 1899; Bronze Medal, and Special Silver Medal for decoration, Paris Exposition, 1900; Bronze Medal, PanAmerican Exposition, Buffalo, 1901. Member Fellowship P. A. F. A.

250. The Blue Coffee Stand.

LOISSEAU, GUSTAVE-Born Paris, France, 1865.

251. La neige a Tournedos.

Loaned by Durand-Ruel \& Sons, New York. 


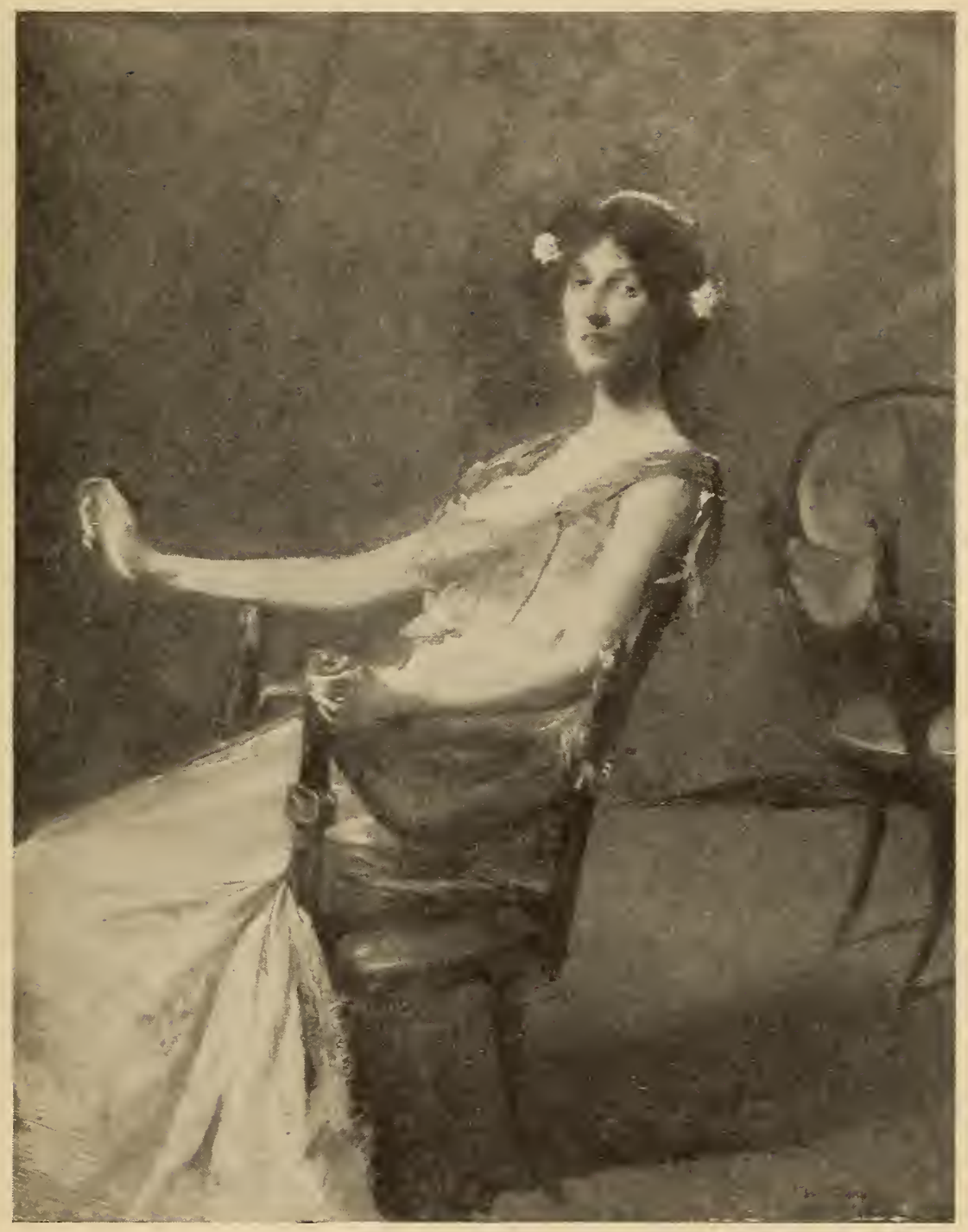

MONTEREY.

Thomas Dewing. 

WOODBURY, CHARLES HERBERT-Born Lynn, Mass., July 14, 1864. Pupil of Mass. Inst. of Technology in Boston; Julian Academy in Paris under Boulanger and Lefebvre. Third Prize, B. A. C.; Gold Medal, Atlanta Exposition, 1895; Second Prize, Tennessee Centennial, Nashville, 1897; Two Medals, Mechanics Fair, Boston; Bronze Medal, Paris Exposition, 1900; Bronze Medal, Pan-American Exposition, Buffalo, 1901; Silver Medal, St. Louis Exposition, 1904; First Prize, Worcester Art Museum, 1903; Honorable Mention, C I., 1905; Second Prize, Worcester, 1907. Member S. A. A., 1899; N. A., 1907; Boston W. C. C. (pres.); N. Y. W. C. C.; St. Botolph C.; Copley S, 1900; Technology C. Specialty, Marines.

252. At Sea.

DIETERLE, MME. MARIE-Born at Sevres, France. Landscape Painter. Daughter and pupil of E. Van Marcke.

253. Pasturage.

Loaned by The Schaus Galleries, New York.

MURPHY, HERMAN DUDLEY-(See 219.)

254. A Summer Morning.

WATROUS, HARRY WILLSON-Born San Francisco, Cal., Sept. 17, 1857. Pupil of Bonnat, Boulanger, Lefebvre and Humphrey Moore in Paris. Clarke Prize, N. A. D., 1894; Bronze Medal, PanAmerican Exposition, Buffalo, 1901; Special Commemorative Gold Medal, St. Louis Exposition, 1904. Member N. A., 1895 (cor. sec.); S. A. A., 1905 ; A. Aid S.; Century Assoc.

255. Some Little Talk There Was of Thee and Me.

ROBINSON, WILLIAM S.-(See 188.)

256. Midsummer Night.

CRANE, BRUCE-Born New York, 1857. Pupil of A. H. Wyant. Webb Prize, S. A. A., 1897; Bronze Medal, Paris Exposition, 1900; George Inness Gold Medal, N. A. D., 1901; Silver Medal, Pan-American Exposition, 1901; Silver Medal, Charleston Exposition, 1902; Gold Medal, St. Louis Exposition, 1904,; N. A., 1901; Member S. A. A., 1881; A. W. C. S.; N. Y. W. C. C.; Salmagundi Club, 1888; Specialty, Landscapes.

257. Golden Autumn.

Loaned by Wm. S. Hurley, Brooklyn, N. Y.

ZANDOMENEGHI, FREDERICO-Born Venice, Italy, 1841.

258. La Modiste.

Loaned by Durand-Ruel \& Sons, New York.

GROLL, ALBERT L.-(See 123.)

259. California Redwoods, Humboldt County. 


\section{Gallery F}

BENSON, FRANK W.-(See 118.)

260. Girl with a Veil.

HOMER, WINSLOW-Born Boston, Mass., 1836. Pupil N. A. D. and F. Rondel; mainly self-taught. First Prize, Pittsburg, C. I., 1896; Gold Medal, P. A. F. A., 1896; Gold Medal, Paris Exposition, 1900; Gold Medal for water color, Pan-American Exposition, Buffalo, 1901; Temple Gold Medal, P. A. F. A., 1902; Gold Medal, Charleston Exposition, 1902; Gold Medal, St. Louis Exposition, 1904. Member N. A., 1865; A. W. C. S.; Century Association; Nat'l. Ins. A. L.

\section{The Fisher Girl.}

Loaned by Burton Mansfield, New Haven, Conn.

MORET, HENRY-(See 214.)

262. La cote Brettone.

Loaned by Durand-Ruel \& Sons, New York.

LIE, JONAS-(See 141.)

263. The Heart of the Woods.

BOUDIN, EUGENE-Born in Honfleur, 1824; died in Deauville, 1898 .

\section{Lever de lune sur la Seine.}

Loaned by Durand-Ruel \& Sons, New York.

DEWING, THOMAS WILMER-Born Boston, Mass., 1851. Pupil of Boulanger and Lefebvre in Paris. Clarke Prize, N. A. D., 1887; Silver Medal, Paris Exposition, 1889; Gold Medal, Pan-American Exposition, Buffalo, 1901; Lippincott Prize, P. A. F. A., 1905; Gold Medal, St. Louis Exposition, 1904; N. A., 1888; Member Ten American Painters. Specialty, Figures.

\section{Monterey.}

MARTIN, HOMER D.--Born at Albany, N. Y., 1836; died, 1897. Pupil of William Hart. Landscape Painter. Elected A. N. A., 1868, and N. A., 1875. Member of the Society of American Artists.

266. On the Seine.

Loaned by Wm. T. Evans, Montclair, N. J. 


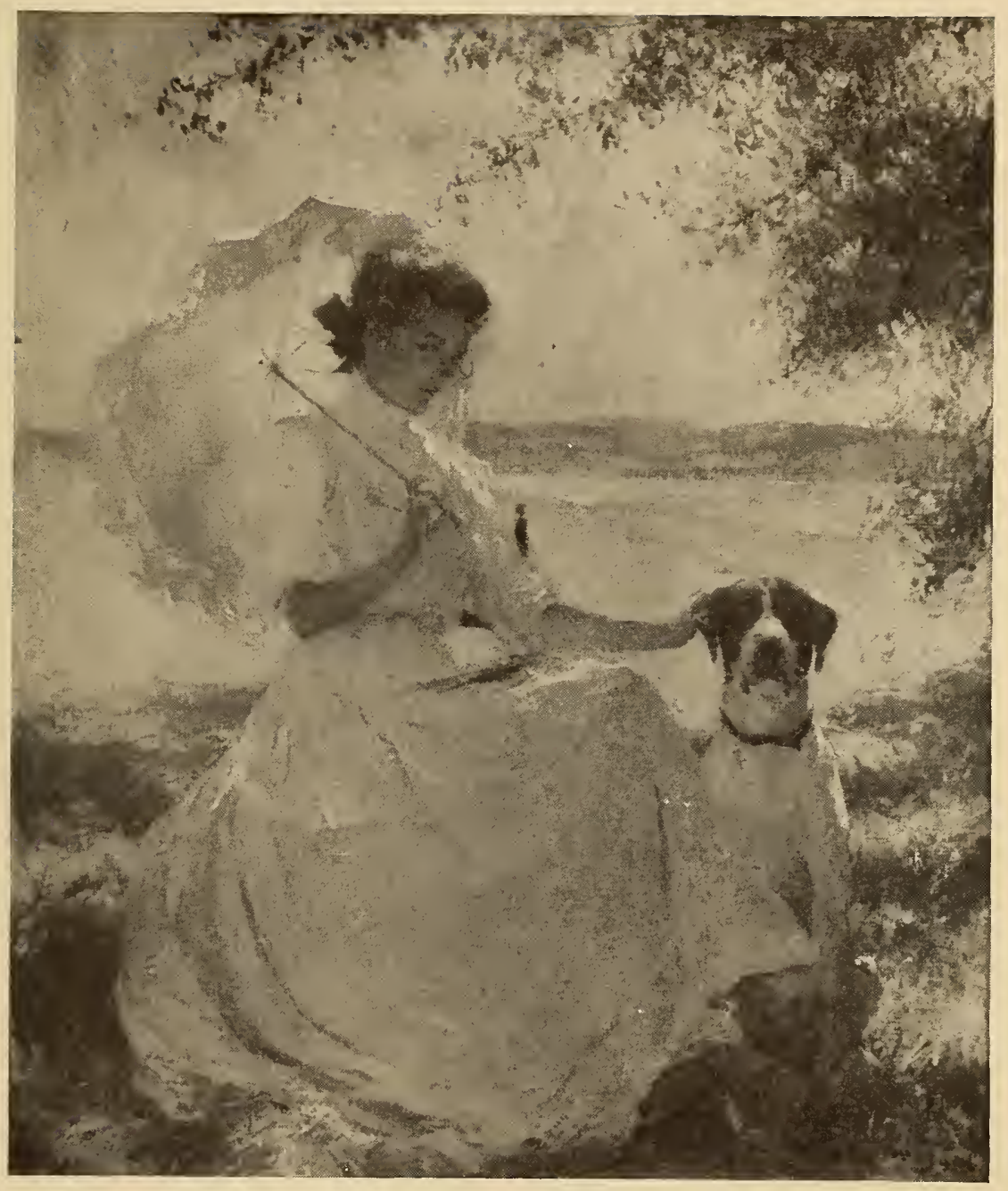

GLIZABETH.

Frank W. Benson. 

KEITH, WILLIAM-Born Aberdeenshire, Scotland, 1839; rame to New York in 1851; engraver until 1859, when he went to California and devoted himself to Landscape Painting. Bronze Medal, PanAmerican Exposition, Buffalo, 1901.

267. Landscape.

Loaned by Wm. S. Hurley, Brooklyn, N. Y.

BLOMMERS, BERNARDUS JOHANNES-Born at The Hague, 1845. Member of the Pulchri Studio, The Hague; Academy of Rotterdam; Royal Scottish Society of Painters in Water Colors, Glasgow. Represented in collections in Amsterdam, The Hague, Rotterdam, Pinakothek, Munich. Bronze Medal, Exposition Universelle, Paris, 1889; Silver Medal, Exposition Universelle, Paris, 1900.

268. Mother and Child.

Loaned by Mrs. C. D. Stimson, Seattle.

HASSAM, CHILDE-(See 122.)

269. Golden Head, Isle of Shoals.

ANDRE, ALBERT-Born in Lyons, France, 1870.

270. Hyde Park.

Loaned by Durand-Ruel \& Sons, New York.

BALLIN, HUGO-Born in New York, 1879. Pupil A. S. L. of N. Y.; studied in Rome. Shaw purchase, S. A. A., 1905; President's Prize, N. Y. Arch Lg., 1907. Clarke Prize, N. A. D., 1906; Second Hallgarten Prize, N. A. D., 1907; Member N. Y. Arch. Lg. 1904; S. A. A. 1905 ; A. N. A. 1906.

271. Three Ages.

MONET, CLAUDE-Born in Paris, 1840. Pupil of Gleyre. Leading Impressionist.

272. La Seine a Lavacour.

Loaned by Durand-Ruel \& Sons, New York.

WEIR, J. ALDEN-(See 129.)

273. Branchviile Landscape.

TWACHTMAN, JOHN H.-(See 106.)

274. October.

Loaned by Silas S. Dustin, New York.

DE HAVEN, FRANK-(See 172.)

275. October Morning.

LOISSEAU, GUSTAVE-(See 251.)

276. Brouillard du matin sur la Seine, 1906.

Loaned by Durand-Ruel \& Sons, New York.

HAWTHORNE, CHARLES W.-(See 115.)

277. La Gigia.

Loaned by William Macbeth, New York. 
ANDRE, ALBERT-(See 270.)

278. Environs de Cannes.

Loaned by Durand-Ruel \& Sons, New York.

SARTAIN, WILLIAM-Born Philadelphia, Pa., 1843. Pupil of P. A. F. A.; Bonnat and Ecole des Beaux Arts in Paris; studied also in Italy and Spain. Silver Medal, Boston; Honorable Mention, P. A. F. A.; Bronze Medal, Pan-American Exposition, Buffalo, 1901; Silver Medal, Charleston Exposition, 1902; A. N. A.; Member S. A. A., 1877.

279. Dunes at Mannasquam, New Jersey.

Loaned by Wiliam Macbeth, New York.

KOOPMAN, AUGUSTUS-(See 250.)

280. Caruluna and Her Duenna.

CHURCH, FREDERIC E.-American School. Born at Hartford, Conn., 1826; died, New York, April 2, 1900 . Pupil of Thomas Cole, at Catskill, where he lived and painted for some years. In 1849 he was elected a member of the N. A. D.; went to South America in 1853, again in 1857. A few years later made studies on the coast of Labrador for his icebergs, which were exhibited in London, 1863. He made studies of Jamacia, 1866; in 1868 he went to Palestine, Greece, and Jerusalem.

281. Sunset on Coast of Maine.

Loaned by Geo. A. Dowden, Newark, N. J.

DAVIES, ARTHUR B.-(See 130.)

282. Our River Hudson.

Loaned by William Macbeth, New York.

CORNOYER, PAUL-Born St. Louis, Mo. Pupil of Lefebvre, Benjamin-Constant and Louis Blane in Paris. First Prize, Paris A. A. A., 1892; Gold Medal, St. Louis Assoc. of Painters and Sculptors, 1895; Evans Prize, Salmagundi Club, 1905; Member Salmagundi Club, 1902.

283. After the Rain, Fifth Avenue, New York.

CRANE, FREDERICK-(See 120.)

284. Nightfall at Dorset.

REHN, FRANK K. M.-Born Philadelphia, Pa., 1848. Pupil of P. A. F. A. First Prize, St. Louis, 1882 ; Prize $(\$ 250)$. Water Color Competitive Exhibition, New York, 1885; Gold Medal, Prize Fund Exhibition, New York, 1886; Honorable Mention, Paris Exposition, 1900 ; Bronze Medal, Pan-American Expositon, 1901; Silver Medal, Charleston Expositon, 1902; Silver Medal, St. Louis Expositon, 1904;. A. N. A.; N. A., 1908 ; Member S. A. A., 1903 ; A. W. C. S.; N. Y. W. C. C.; Salmagundi Club, 1883 Specialty, Marines

285. Manchester by the Sea. 


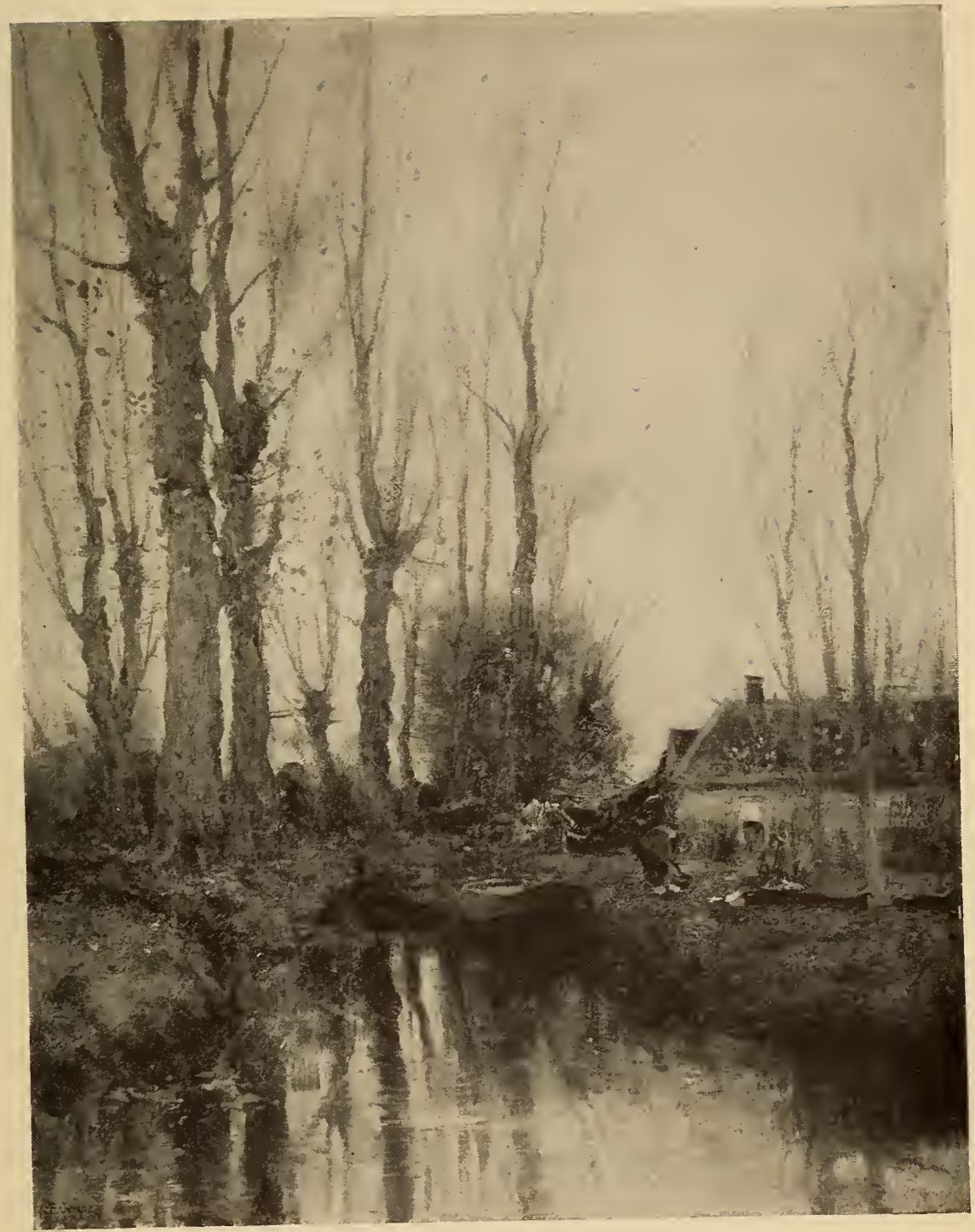

NOVEMBER.

Charles P. Gruppe. 

MAUFRA, MAXIME-Born Nantes, France, 1861.

286. Nuit lunaire-Vancottes sur mer.

Loaned by Durand-Ruel \& Sons, New York.

SARTAIN, WILLIAM-(See 279.)

287. A Marsh Pool.

GENTH, LILLIAN M.-(See 110.)

288. Maidenhood.

MONET, CLAUDE-(See 272.)

289. Les saules.

Loaned by Durand-Ruel \& Sons, New York.

HASSAM, CHILDE-(See 122.)

290. The Oasis-Twilight Moonlight.

BALLIN, HUGO-(See 271.)

291. The Lesson.

OCHTMAN, LEONARD-(See 203.)

292. Moonset.

BENSON, FRANK W.-(See 118.)

293. Elizabeth.

COOPER, COLIN CAMPBELL-(See 242.)

294. The Church of St. Wolfram, Abbeville, France.

CRANE, BRUCE--(See 257.)

295. Departing Winter.

DE HOOG, BERNARD.

296. By the Fireside.

Loaned by R. C. \& N. M. Vose, Boston.

DOUGHERTY, PAUL-(See 168.)

297. Pirates' Cove.

WIGGINS, CARLETON-Born at Turner's, Orange Co., N. Y., March 4, 1848. Pupil of N. A. D. and George Inness in New York. Gold Medal, Prize Fund Exhibition, New York, 1877; Bronze Medal, Pan-American Exposition, Buffalo, 1901; Member International Jury of Awards, St. Louis Exposition, 1904; N. A., 1906; Member S. A. A., 1887; A. W. C. S.; S. Landscape P.; Salma. C., 1898; Brooklyn A. C.; A. Fund S.; A Aid S. Specialty, Animals.

298. October Morning on the Hill. 
EATON, CHARLES WARREN-(See 108.)

299. The Night.

HUGUET, VICTOR PIERRE-(See 138.)

300. Caravane en Algerie.

Loaned by Durand-Ruel \& Sons, New York.

WIGGINS, CARLETON-(See 298.)

301. A Holstein Cow.

BOUDIN, EUGENE-(See 264.)

302. Un Grain du Nord-Ouest Deauville.

Loaned by R. C. \& N. M. Vose, Boston.

MOORE, ERNEST-(See 212.)

303. Right Hon. James Bryce.

HAWTHORNE, CHARLES W.-(See 115.)

304. Venetian Girl.

BEAL, GIFFORD-(See 226.)

305. The Ruins of Glenham.

ZANDOMENEGHI, FREDERICO-(See 258.)

306. Dos de femme.

Loaned by Durand-Ruel \& Sons, New York.

KENSETT, JOHN FREDERICK-Born Cheshire, Conn., 1818; died, New York, 1872. Landscape Painter. Studied engraving under Dagget. Exhibited at the Royal Academy, London, in 1845 . Elected N. A., 1849.

307. Landscape.

Loaned by Wm. S. Hurley, Brooklyn, N. Y.

MAUFRA, MAXIME-(See 286.)

308. La ville feerique-Exposition Universelle, Paris, 1900. Loaned by Durand-Ruel \& Sons, New York.

BRECKENRIDGE, HUGH H.-(See 232.)

309. Blue and Gold.

OCHTMAN, LEONARD-(See 203.)

310. A Moonlight Fantasy.

HENRI, ROBERT-(See 128.)

311. Rocks and Sea. 


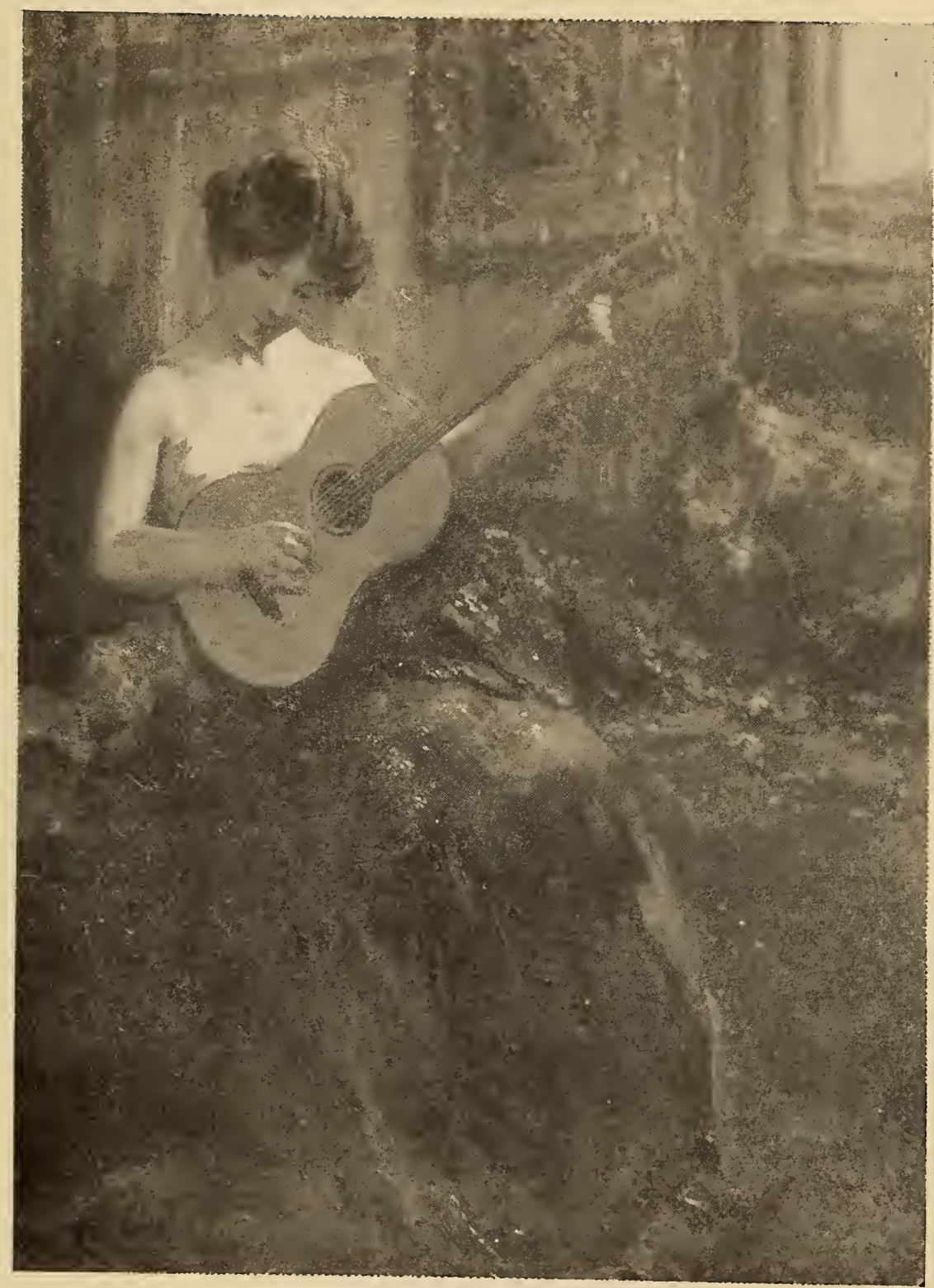

HARMONY

Chas. P. Gruppe 



\section{Upper Atrium}

D'ESPAGNAT, GEORGES-(See 223.)

312. Le Soir sur la Seine.

Loaned by Durand-Ruel \& Sons, New York.

NETTLETON, WALTER-(See 248.)

313. Frozen Fields.

WIEGAND, GUSTAVE-(See 195.)

314. The Oaks.

WOODBURY, CHARLES H.-(See 252.)

315. Gulf Stream.

SCHREYVOGEL, CHARLES-Born New York, Jan. 4, 1861. Pupil of August Schwabe in Newark, N. J.; Carl Marr, Frank Kirchbach in Munich. Clarke Prize, N. A. D., 1900; Bronze Medal, Paris Exposition, 1900; Bronze Medal, Pan-American Exposition, Buffalo, 1901; Bronze Medal, St. Louis Exposition, 1904. A. N. A., 1901; Member Salma. C., 1902.

316. Ereaking Through the Circle.

Loaned by Mrs. Kate Linde, Hoboken, N. J.

MOORE, ERNEST-(See 212.)

317. A Breeze of Morning Moves.

DUPRE, JULIEN-Born at Paris, 1851. Pupil of Pils, Laugee and Lehmann. Honorable Mention, 1879. Medals; Third Class, Exposition Universelle; Gold Medals, 1890, Munich and Antwerp, 1894. Legion of Honor, 1892 .

318. The Coming Storm.

Loaned by Prinz Bros., Brooklyn, N. Y. 


\section{Gallery G}

GRUPPE, CHARLES P.-(See 179.)

319. November.

SMITH, W. GRANVILLE-(See 185.)

320. Marine.

THOMAS, GROSVENOR-Glasgow school. Member of the International Society of Sculptors, Painters and Gravers, and the Pastel Society, London; Royal Scottish Society of Painters in Water Colors; and Glasgow Institute of Fine Arts. Represented in the National Gallery, Budapest; National Gallery of Bohemia, Prague; and Albright Art Gallery, Buffalo. Gold Medal, Dresden, 1901; Medal of the Second Class, International Exposition, Munich, 1901.

321. The Berid of the River.

Loaned by The Albright Art Gallery, Buffalo.

YATES, CULLEN-(See 147.)

322. Rural Life.

A LF.XANDER, J. W.-( See 237.)

323. A Mother.

TANNER, HENRY OSSAWA-(See 166.)

324. The Good Shepherd.

WYANT, ALEXANDER H.-Born Port Washington, O., 1836; died, New York, 1892. Landscape painter. Studied under Hans Gude in Karlsruhe. Student of the works of Turner and Constable in London. Elected A. N. A., 1868; N. A., 1869.

325. An Adirondack Vista.

Loaned by Wm. T. Evans, Montclair, N. J.

BOGERT, GEORGE H.-(See 163.)

326. Approaching Storm. 


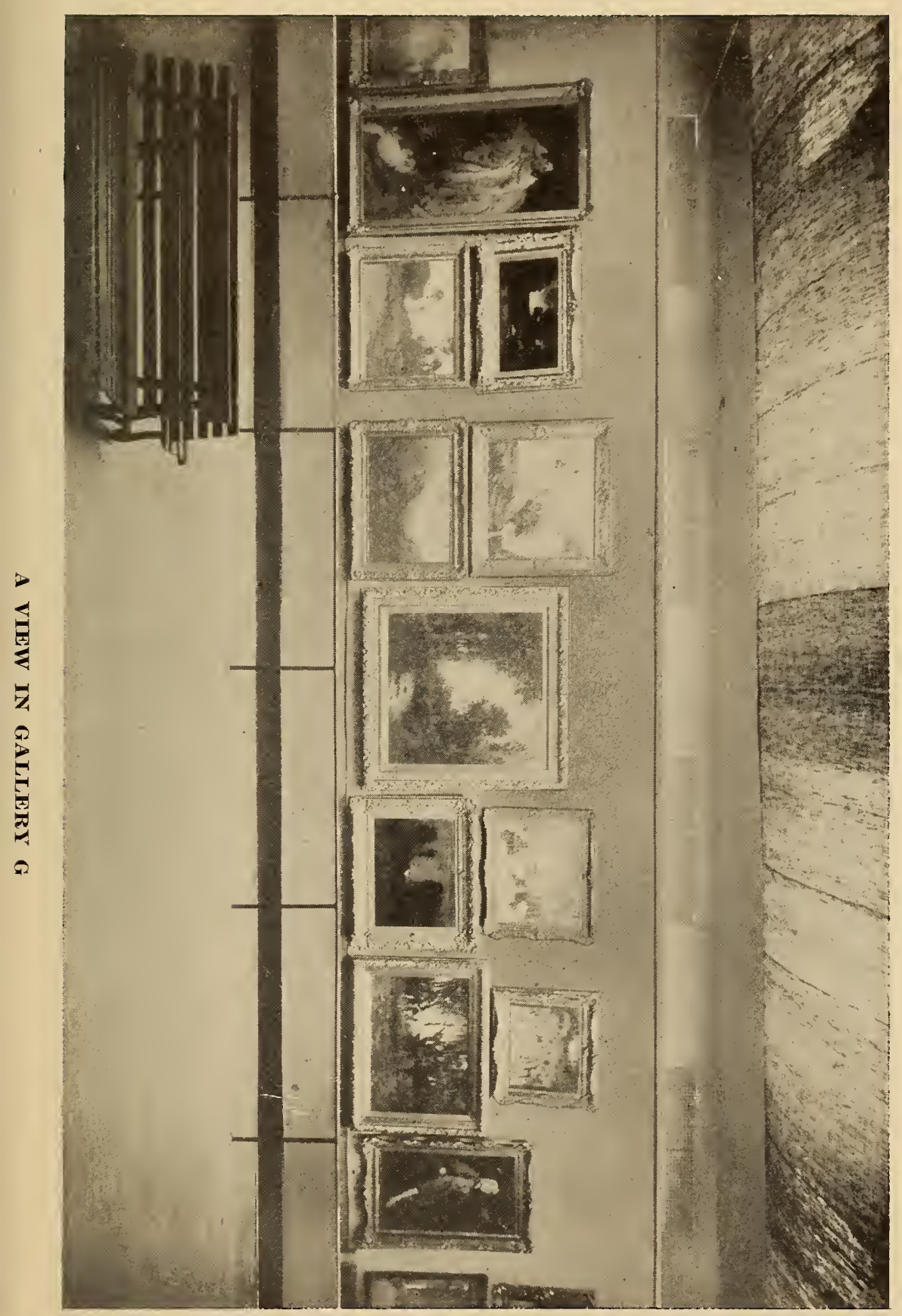



GRUPPE, CHARLES P.-(See 179.)

327. Harmony.

BOGERT, GEORGE H.-(See 163.)

328. Glowing Venice.

Loaned by The Blakeslee Galleries, New York.

OCHTMAN, LEONARD-(See 203.)

329. Autumn Days.

DE HAVEN, FRANK-(See 172.)

330. Autumn.

REDFIELD, EDWARD W.--(See 169.)

331. The Canal, Evening.

ROBINSON, WILLIAM S.-(See 188.)

332. In the Meadow.

WILES, IRVING R.-(See 204.)

333. Portrait in Black.

TWACHTMAN, JOHN H.-(See 106.)

334. Niagara.

BUNCE, WILLIAM GEDNEY-Born Hartford, Conn., 1840; pupil of Cooper Union and Wm. Hart in New York; Achenback and P. J. Clays in Antwerp. Bronze Medal, Paris Exposition, 1900; Silver Medal, Pan -American Exposition, Buffalo, 1901; Silver Medal, Charleston Expositon, 1902; Silver Medal, St. Louis Exposition, 1904. N. A., 1907 ; Nat. Inst. A. L.

335. Venice.

Loaned by Wm. T. Evans, Montclair, N. J.

WILLIAMS, FREDERICK BALLARD-(See 165.)

336. The Court of the Beloved.

VOLK, DOUGLAS-Born Pittsfield, Mass., Feb. 23, 1856. Pupil of Gerome in Paris. Medal, Columbian Exposition, Chicago, 1893; Shaw Fund Purchase, S. A. A., 1899; First Prize, Colonial Exhibition, Boston, 1899; Silver Medal, Pan-American Exposition, Buffalo, 1901; Silver Medal, Charleston Exposition, 1902; Carnegie Prize, S. A. A., 1903; Silver Medal, St. Louis Exposition, 1904. Member N. A., 1899 ; S. A. A., 1880 ; N. A. C.; A. Fund S.; A. Aid S. Instructor at Cooper Union. Organized handicraft movement at Centre Lovell.

337. Maid of the Manor. 
RANGER, HENRY W.-(See 146.)

338. Getting Ship Timber.

Loaned by William Macbeth, New York.

NEWELL, GEORGE GLENN-Born Berrian Co., Mich., 1870. Pupil of N. A. D., under Ward; Teachers College, New York, under Will S. Robinson. Member Salmagundi Club, 1898; Country Sketch C. Specialty, Landscapes and Cattle.

339. Through Shower and Sun.

BALLIN, HUGO-(See 271.)

340. Susanna and the Elders.

NEWELL, GEORGE GLENN-(See 339.)

341. When the Moon Gives Light.

EATON, CHARLES WARREN-(See 108.)

342. Pines at Knocke.

BENSON, FRANK W.-(See 118.)

343. Woman Reading.

MAUVE, ANTON-Dutch; 1838-1891. Represented in the $\mathrm{Mu}$ seums at the Hague, Amsterdam, and Rotterdam.

344. Goats Feeding. Loaned by Mrs. C. D. Stimson, Seattle.

SLOANE, GEORGE.

345. The Chess Players.

Loaned by R. C. \& N. M. Vose, Boston.

INNESS, GEORGE-(See 164.)

346. Summer-Montclair.

Loaned by R. C. \& N. M. Vose, Boston.

SMITH, W. GRANVILLE-(See 185.)

347. Arabian Nights.

CARLSEN, EMIL-(See 189.)

348. In the Woods.

Loaned by Mrs. Kate Linde, Hoboken, N. J.

VOLK, DOUGLAS-(See 337.)

349. Thoughts of Youth.

GRUPPE, CHARLES P.-(See 179.)

350. Milking Time. 


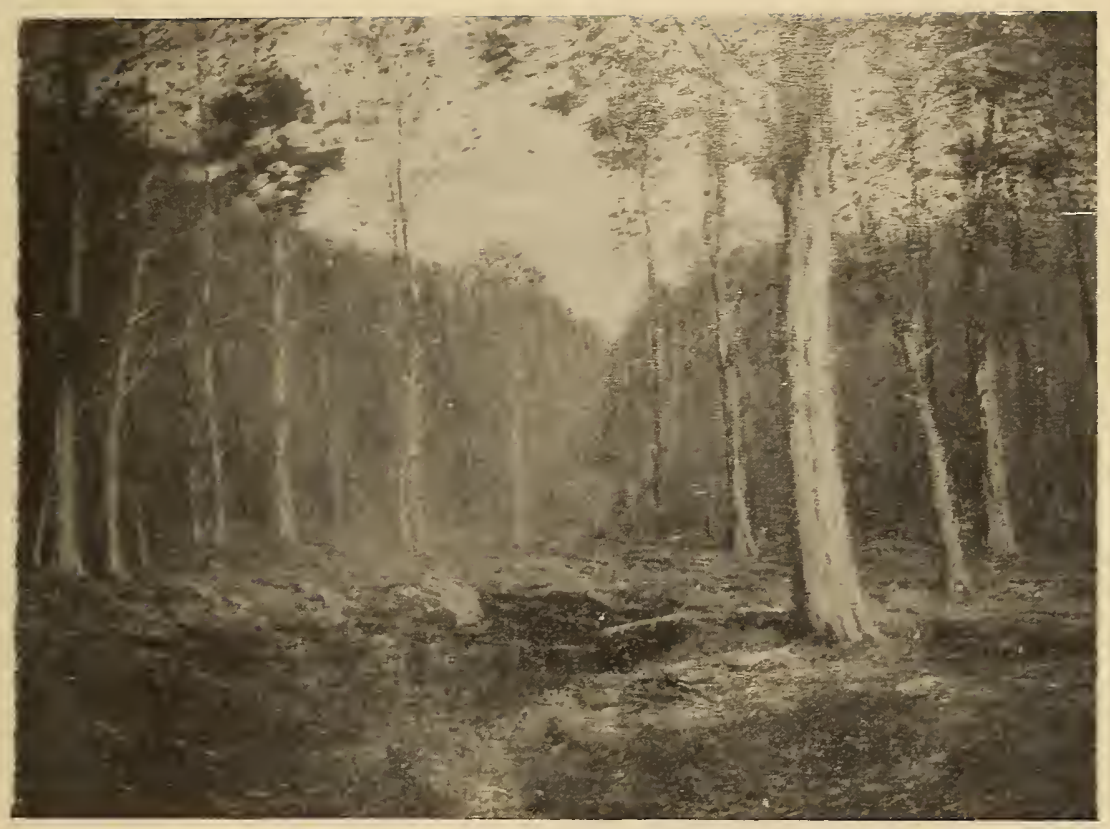

AUTUMN DAYS

Leonard Ochtman 

COUSE, E. IRVING-(See 121.)

351. A Young Herder.

DEARTH, HENRY GOLDEN-(See 197.)

352. Moonlight.

Loaned by The Folsom Co., New York.

KOOPMAN, AUGUSTUS-(See 250.)

353. A Venetian Tratorria.

WYANT, ALEXANDER H.-(See 325.)

354. In the Still Woods.

Loaned by Mrs. A. L. Wyant, New York.

WILLIAMS, FREDERICK BALLARD-(See 165.)

355. Hills of Purple and Rose.

LOEB, LOUIS-Born in Cleveland, O., 1866. Pupil of Gerome in Paris. Honorable Mention, Paris Salon, 1895; Third Class Medal, Paris Salon, 1897; Silver Medal for drawing and Silver Medal for painting, Pan-American Exposition, Buffalo, 1901; Hallgarten Prize, N. A. D., 1902; Webb Prize, S. A. A., 1903; Silver Medal for painting and Silver Mredal for illustration at the St. Louis Exposition, 1904; Carnegie Prize, S. A. A., 1905; A. N. A., 1901; N. A., 1906; Nember S. A. A., 1900; New York Arch. Lg., 1902; S. I. Also Teacher.

356. The Summit.

RYDER, CHAUNCEY F.-(See 127.)

357. Autumn Clearing.

BLAKELOCK, RALPH A.-(See 174.)

358. Ghost Dance.

Loaned by Wm. S. Hurley, Brooklyn, N. Y.

1.UBBELL, HENRY S.-Born in Paola, Kansas, 1870. Pupil of A. I. C.; Whistler, Collin, Laurens and Benjamin-Constant in Paris. Honorable Mention, Paris Salon, 1901; Third Class Medal, Paris Salon, 1904; Silver Medal, St. Louis Exposition, 1904; Third Prize, Worcester, (Mass.) Museum, 1905. Member Paris A. A. A.; S. A. A., 1905 ; Paris S. A. P.; A. N. A., 1906; S. W. A.

359. Caprice.

SMITH, W. GRANVILLE-(See 185.)

360. The Birches.

MURPHY, J. FRANCIS-(See 161.)

361. The Pond.

Loaned by William Macbeth, New York. 
362. The Golden Hour.

BRUSH, GEORGE DE FOREST-Born Shelbyville, Tenn., Sept. 28, 1855. Pupil of Gerome. First Hallgarten Prize, N. A. D. 1888; Medal, Columbian Exposition, Chicago, 1893; Temple Gold Medal, P. A. F. A., 1897; Gold Medal, Paris Exposition, 1900; Gold Medal, Pan-American Exposition, Buffalo, 1901; Gold Medal, St. Louis Exposition, 1904; Saltus Medal, N. A. D., 1909. N. A., 1906; Member S. A. A., 1880; A. Fund S.; Nat. Inst. Ä. L.

363. The Weaver.

Loaned by J. M. Shipman, Newark, N. J.

WALKER, HORATIO-Born in Canada, 1858. Evans prize, A. W. C. S., 1888; Gold Medal, Competitive Exposition at American Art Galleries, N. Y., 1887; Bronze Medal, Paris Exposition, 1889; Gold Medal and Diploma, Columbian Exposition, Chicago, 1893; Gold Medal, Pan-American Exposition, Buffalo, 1901; Gold Medal, Charleston, 1902; Gold Medal for Oil and Gold Medal for Water Colors, St. Louis Exposition, 1904; Gold Medal of Honor, P. A. F. A., 1906; First Prize, Worcester, 1907 . Member N. A., 1891; 'S. A. A., 1887 ; Nat. Inst. A. L.; Royal Inst. of Painters in Water Colors, England; A. W. C. S.; A. Fund S.; A. Aid S.

364. Potato Gatherers.

Loaned by Burton Mansfield. New Haven, Conn.

DEWING, THOMAS-(See 265.)

365. The Letter.

CARLSEN, EMIL-(See 189.)

-366. Still Life.

- 367. The Rising Storm.

Loaned by Wm. S. Hurley, Brooklyn, N. Y.

Loaned by Mrs. Kate Linde, Hoboken, N. J.

WALKER, HORATIO-(See 364.)

368. The Thresher.

Loaned by A. T. Sanden, Goshen, N. Y.

LENBACH, FRANZ VON-Born in Bavaria, 1836; died in Munich, 1904. Pupil of Munich Royal Academy, Grafle and Piloty. Medal of Paris Salon, 1867. Grand Prix, E. U., 1900. Member of Royal Academy, Berlin.

369. Mrs. T.

Loaned by Harry Whitney Treat, Seattle.

WEIR, J. ALDEN-(See 129.)

370. Ploughing for Buckwheat.

HUBBELL, HENRY S.-(See 359.)

371. Henry and Jack. 


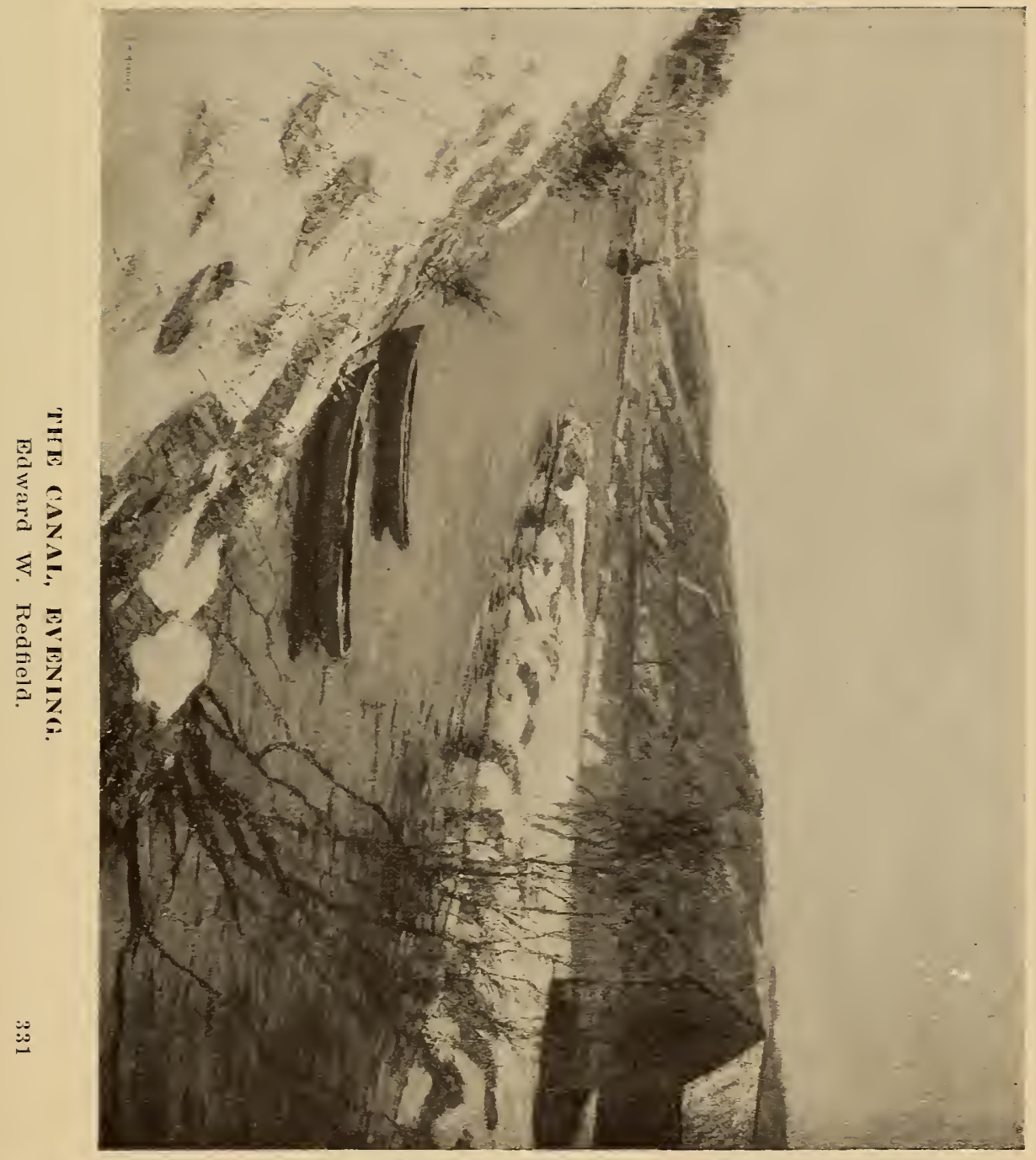





\section{Gallery $\mathrm{H}$}

COURBET, GUSTAVE-(See 74.)

372. After the Storm.

Loaned by Judge C. H. Carey, Portland, Ore.

DIAZ, NARCISSE VIRGILE-(See 3.)

373. Figures.

Loaned by Mrs. David 'T. Honeyman, Portland, Ore.

GOSSELIN, ALBERT-French. Honorable mention, 1890; third Class Medal, 1896; Second Class, 1897; Gold Medal, 1900, (E. U.;) Legion d'Honneur, 1900; Member of Jury. Paintings in Luxembourg.

374. Landscape.

Loaned by W. B. Ayer, Portland, Ore.

BOUDIN, EUGENE-(See 264.)

375. Marine.

Loaned by C. E. S. Wood, Portland, Ore.

COROT, J. B. C.-(See 2.)

376. Landscape.

Loaned by C. E. S. Wood, Portland, Ore.

MONTICELLI, ADOLPHE-French; 1824-1886. Pupil of Academy of Fine Arts, Marseilles, and of Delacroix and Diaz, in Paris.

377. Figures.

Loaned by C. E. S. Wood, Portland, Ore.

GAGLIARDINI, JULIEN GUSTAVE-French. Honorable Mention, 1883; Medals: 1884, 1886, 1889, (E. U.) Legion d'Honneur, 1893; Gold Medal, 1900, (E. U.) Paintings in Luxembourg.

\section{La Riviere.}

Loaned by W. H. Drant, Paris. 
RIEDER, M.-French. Medal, Third Class, 1898; Second Class, 1899; Medal, 1900 (E. U.)

379. Bon Soir.

Loaned by A. S. Kerry, Seattle.

MONTICELLI, ADOLPHE-(See 377.)

380. By the Lake-Feeding Cat.

Loaned by Mrs. F. S. Stimson, Seattle.

BARILLOT, LEON-French. Medal Third Class, 1880; Second Class, 1884; Medaille d'Or, 1889 (E. U.); Legion d'Honneur, 1885; Member of Jury; Gold Medal, 1900, (E. U..). Paintings in Luxembourg.

381.

Loaned by Mrs. F. S. Stimson, Seattle.

ROBERT-FLEURY, TONY-Born at Paris, 1837. Medals, 1866, 1867, 1870; Medal of Honor, 1870; Legion d'Honneur, 1873; Officier, 1884; Medal, First Class, 1878, (E. U.); Medaille d'Or, 1899, (E. U.); Member of Jury; H. C., 1900 ; (E. U.); Commander Legion d'Honneur, 1907 .

382. The Reader.

Loaned by Mrs. F. S. Stimson, Seattle.

BUFFET, AMEDEE-French. Honorable Mention, 1894; Third Class Medal, 1898; Second Class Medal, 1900.

383. Evening in the South of France.

Loaned by Mrs. F. S. Stimson, Seattle.

HARPIGNIES, HENRI-Born at Valenciennes, 1819. Medals, 1866, 1868, 1869; Legion d'Honneur, 1875; Second Class Medal, 1878 (E. U.); Officer of the Legion d'Honneur, 1875; Second Class Medal 1878 (E. U.) ; - Medal of Honor, 1897; Grand Prix, 1900 (E. U.;) Commander of the Legion of Honor, 1901.

384. Le Soir.

Loaned by Mrs. F. S. Stimson, Seattle.

DELACHAUX, LEON-French. Medal, Exhibition, 1900. Member Societie Nationale. Paintings in Luxembourg.

385. Congratulating the Fiance-Brittany.

Loaned by A. S. Kerry, Seattle.

GOSSELIN, ALBERT-(See 374.)

386. The Road to the Woods.

Loaned by Mrs. A. S. Kerry, Seattle. 


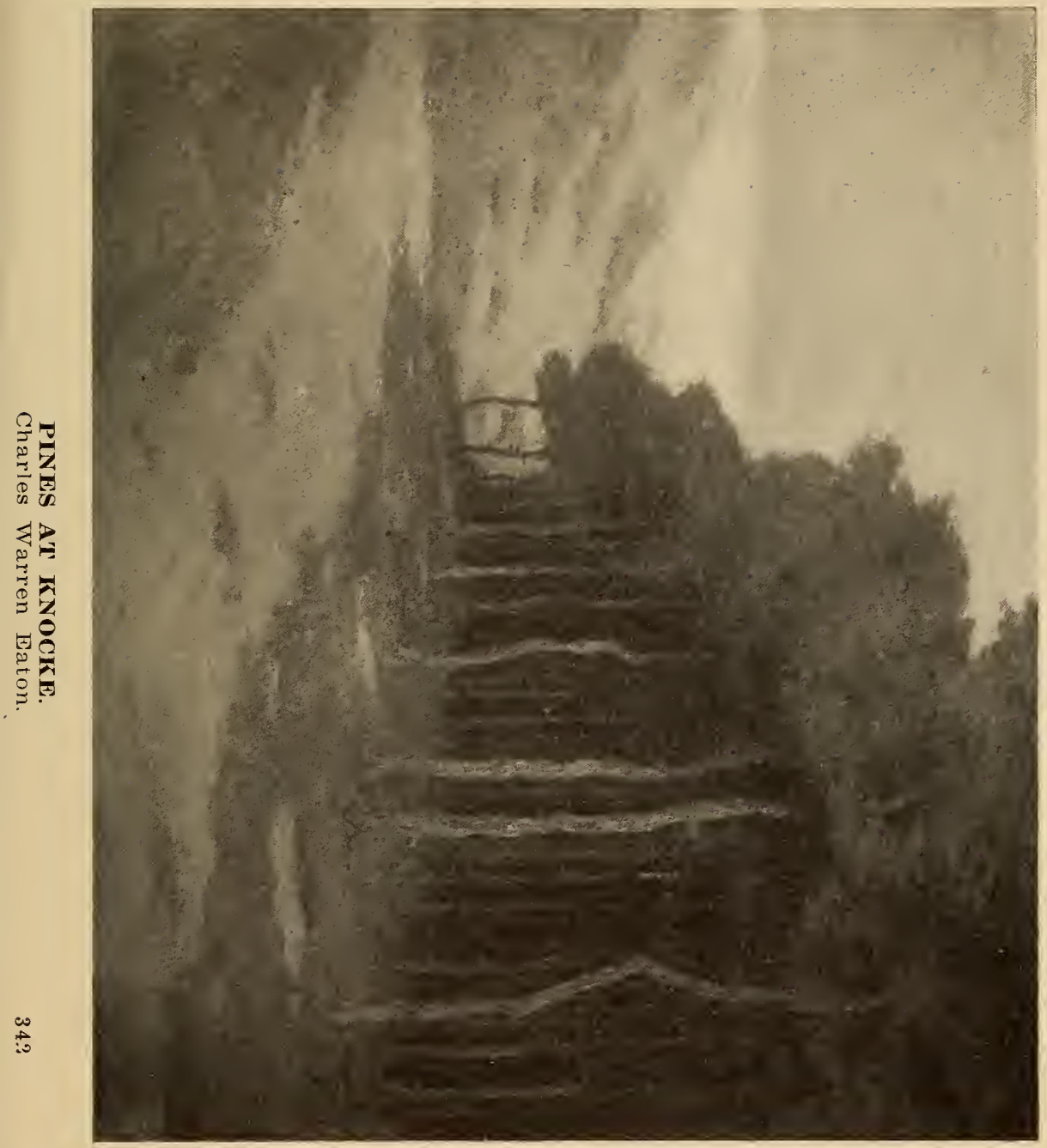



BUFFET, PAUL-French. Third Class Medal, 1890; Second Class, 1893; Bourse du Voyage, 1894; Prize Nationale, 1896; Medaille d'Or, 1900. Painting in Luxembourg.

387. Landscape.

Loaned by Mrs. A. S. Kerry, Seattle.

BARILLOT, LEON-(See 381.)

388. Marais de Berniers-matin.

Loaned by A. S. Kerry, Seattle.

BUFFET, PAUL-(See 387.)

389. Landscape.

Loaned by Mrs. P. F. Kelley, Seattle.

ISABEY, EUGENE-French; 1804-1886. Son and pupil of Jean Baptiste Isabey. Medals, 1824, 1\$27, 1\$55. Chev. Legion d'Honneur, 1832. Officier, 1852.

390. Marine.

Loaned by Mrs. A. S. Kerry, Seattle.

SOUSA-PINTO, JOSE-Born at Terceira, Portugal, 1857. Pupil of Cabanel. Honorable Mention, 1883; Medal First Class, Nice, 1894; Medal Second Class, 1S\$9, (E. U.); Legion d'Honneur, 1895; Grand Prix, Portugal, 1898; Medal, 1900, (E. U.)

391. Boy Roasting Potatoes.

Loaned by Mrs. A. S. Kerry, Seattle.

GAGLIARDINI, JULIEN GUSTAVE-(See 378.)

392. Village Road-South of France.

ROUSSEAU, THEODORE-(See 16.)

393. The Wood Road.

Loaned by J. W. Clise, Seattle.

COROT, J. B. C.-(See 2.)

394. Shepherd in the Roman Country.

Loaned by J. W. Clise, Seattle.

PELOUSE, LEON GERMAIN-French; 1838-1891. Medal of the Second Class, 1873; Medal First Class, 1876; Medal Second Class, 1878 , (E. U.); Legion d'Honneur, 1S78; Medal, First Class, 1889 (E. U.)

395. After the Rain.

Loaned by J. W. Clise, Seattle. 
DIAZ, NARCISSE VIRGILE-(See 3.)

396. Interior-Forest of Fontainebleau.

Loaned- by J. Wr. Clise, Seattle.

GOSSELIN, ALBERT-(See 374.)

397. Le Loing a Grez.

Loaned by Mrs. C. F. White, Seattle.

HARPIGNIES, HENRI-(See 384.)

398. Le Saut de Laup.

Loaned by Dr. Alfred Raymond, Seattle.

RIGOLOT, ALBERT GABRIEL-French. Honorable Mention, 1889; Medal, Third Class, 1891; Second Class, 1892; Gold Medal, 1894, (E. U.); Lyons and Paris, 1900; Legion d'Honneur, 1901. Paintings in Luxembourg.

399. Le Soir.

Loaned by Frank McDermott, Seattle.

ZUBER, JEAN HENRI-French. Medal, Third Class, 1875; Second Class, 1878 (E. U.); Legion d'Honneur, 1886; Gold Medal, 1889 (E. U.); Gold Medal, 1900 (E. U.); Officier Legion of Honor, 1906. Paintings in Luxembourg.

400. Evening by the Pond.

Loaned by Mrs. H. R. Clise, Seattle.

JAPY, LOUIS-French. Medal, Third Class, Salon, Paris, 1870; Second Class, 1873; Medaille d'Argent, 1889, (E. U.)

401. Landscape.

Loaned by M. H. Walker, Salt Lake City, Utah.

DELACHAUX, LEON-(See 385.)

402. Family-Interior.

Loaned by C. J. Smith, Seattle.

BARILLOT, LEON-(See 381.)

403.

WAGREZ, JACQUES CLEMENS-French. Honorable Mention, 1878; Medal, Third Class, 1879; Second Class, 1898; Gold Medal, 1900. (Deceased.)

404. Fortune Teller.

Loaned by M. H. Walker, Salt Lake City, Utah. 


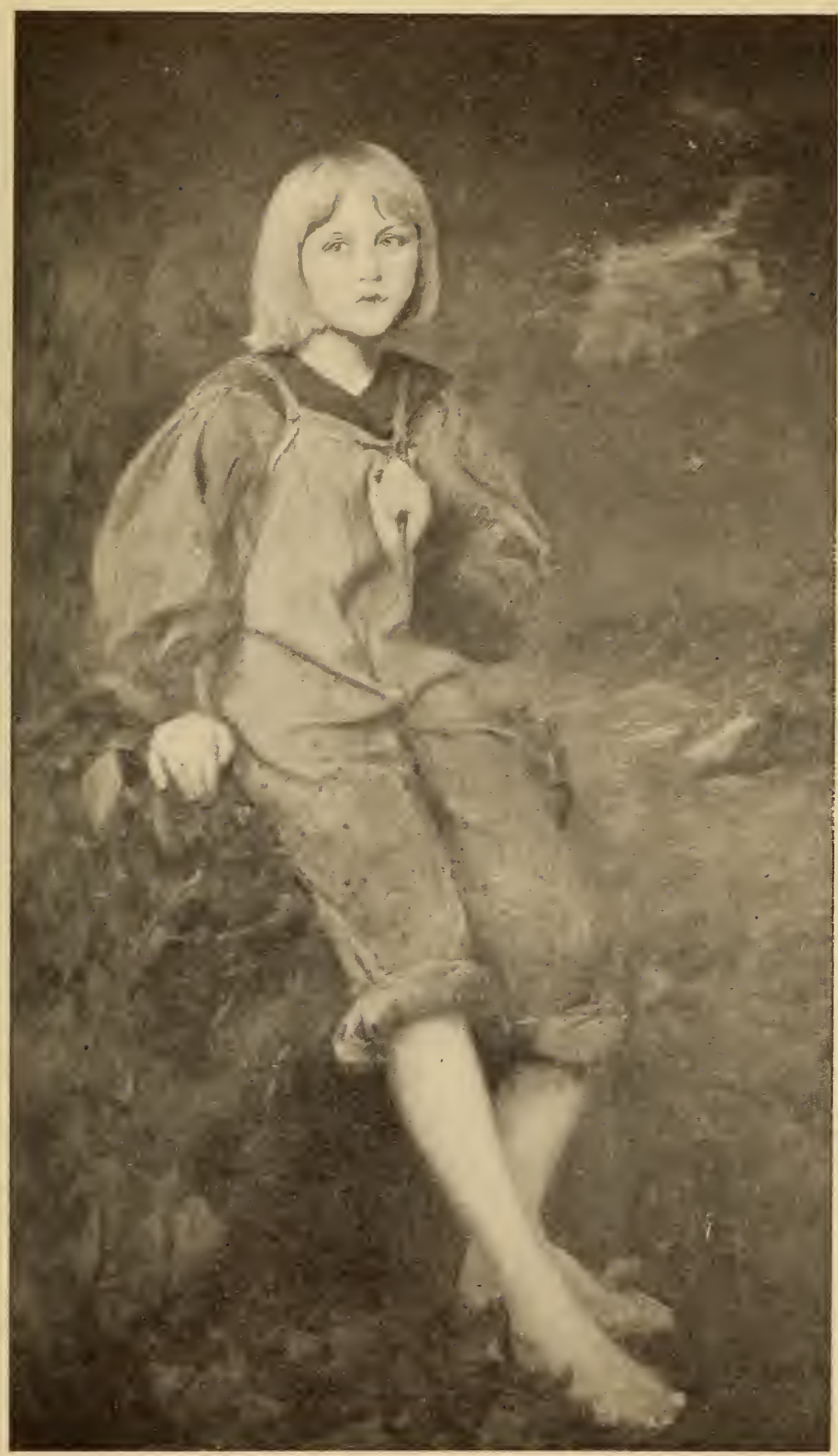

THOCGHTS OF YOUTH

Douglas Volk 

ZUBER, JEAN HENRI-(See 400.)

405. Landscape.

Loaned by Frank McDermott, Seattle.

CHRETIEN, RENE LOUIS-French. Honorable Mention, 1889; Medal Third Class, 1894; Second Class, 1895; Medaille d'argent, 1900 , (E. U.)

406. The Lessons.

Loaned by M. H. Walker, Salt Lake City, Utah.

PELOUSE, L. G.-(See 395.) .

407. The Vineyard.

Loaned by W. H. Drant, Paris.

408. Landscape.

Loaned by W. H. Drant, Paris.

409. Morning Dew at Rochefort.

Loaned by A. L. White, Spokane, Wash.

BARILLOT, LEON-(See 381.)

410.

CHARPIN, ALBERT-French. Honorable Mention, 1883; Medal, Third Class, 1893; Second Class, 1897.

\section{Shepherdess of Provence.}

Loaned by C. F. White, Seattle.

CHABAS, PAUL EMILE-French. Honorable Mention, 1892; Medal Third Class, 1895; Second Class, 1896; Prize Nationale, 1899; Gold Medal, 1900. (E. U.) Legion d'honneur, 1902. Paintings in Luxembourg.

412. The E.athers.

Loaned by M. H. Walker, Salt Lake City, Utah.

WALLET, ALBERT CHARLES-French. Honorable Mention, 1884; Medal, Third Class, 1893; Second Class, 1895; Medal, Second Class, 1900 (E. U.)

413.

PERRAULT, LEON-French. Medals: Paris, 1864, 1865; Philadelphia Centennial Exposition, 1876. Legion d'Honneur, 1877. Medal, 1889, (E. U.) Paintings in Luxembourg. (Deceased.)

414. Maternal Love.

Loaned by M. H. Walker, Salt Lake City, Utah. 
RAVANNE, LEON GUSTAVE-French. Honorable Mention, 1887; Bronze Medal, 1892, (E. U.); Medal, Second Class, 1595; Medal, 1900 , (E. U.) Paintings in Luxembourg. (Deceased.)

415. Marine.

Loaned by M. H. Walker, Salt Lake City, Utah.

GUILLOU, ALFRED-

416. Girls Fishing.

Loaned by W. H. Drant, Paris.

BARILLOT, LEON-(See 3S1.)

417.

PASINI, ALBERTO-French; 1826-1899. Pupil of Isabey and Th. Rousseau. Mcdals at Salon, $1 \$ 59,1863,1864$; Legion d'Honneur, 1868 ; Officier, 1878 .

418. Pasha's Escort.

Loaned by IV. H. Drant, Paris.

PERRAULT, LEON-(See 414.)

419. Marguerite.

L.oaned by C. H. Frye, Seattle.

WALLET, ALBERT CHARLES-(See 413.)

420.

TATTEGRAIN, FRANCIS-French. Honorable Mention, 1881. Medal, Second Class, 1883; Gold Medal, 1889, (E. U.); Legion d'Honneur, 1889; Medal of Honor, Salon, 1899; Gold Medal, 1900, (E. U.) Paintings in Luxembourg.

421. Distress Anchorage-Cape Grisnez.

Loaned by W. H. Drant, Paris.

GRIMLUND, JOHANNES-French. Honorable Mention, 1884; Medal Third Class, 18ss; Bronze Medal, 1889, (E. U.;) Legion d'Honneur, 1\$92; Medal, 1900, (E. U.)

422. Fishing Village.

Loaned by. W. H. Drant, Paris.

MONTICELLI, ADOLPHE-(See 377.)

423. Confidence.

Loaned by W. H. Drant, Paris. 


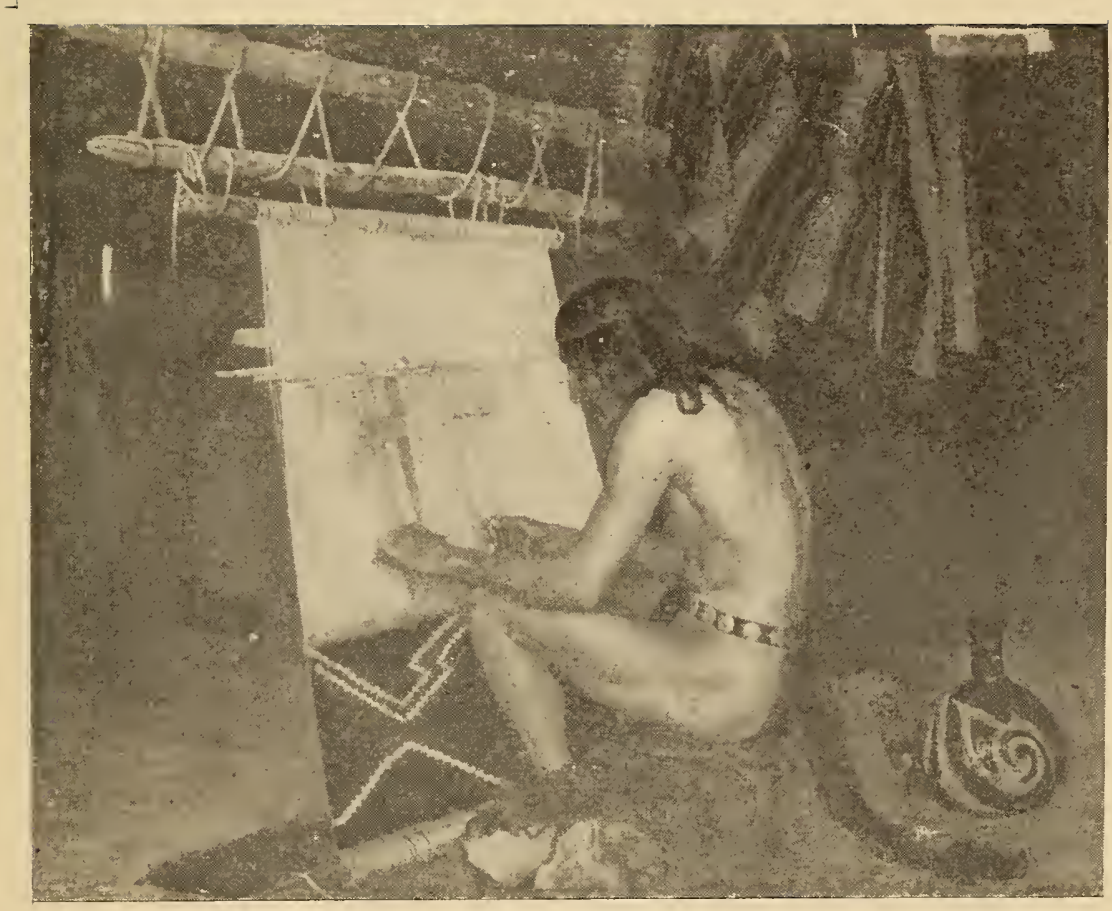

THE WEAVER

George De Forest Brush 



\section{Sculpture}

EBERLE, ABASTENIA ST. LEGER-Born in Iowa, 1878. Pupil of George Gray Barnard, Gutzon, Borglum, A. S. L. of N. Y. Bronze MIedal, St. Louis Exposition, 1904 . Member N. S. S.; N. Y. Woman's A. C.
500. The Dancer.
501. The Siren.
502. Grief.
503. The Coal Picker.
504. Indian Fighting Eagle.

EVANS, RUDOLPH-Born Washington, D. C. Pupil of Falguiere and Peuch. Member Paris A. A. A.

505. Bronze Head of Miss Maude Adams.

FRENCH, DANIEL CHESTER-Born Exeter, N. H., 1850. Pupil of Thomas Ball. Third Class Medal, Paris Salon, 1892; Grand Prize of Honor, Paris Exposition, 1900; Nember International Jury of Awarđ̄s, St. Louis Exposition, 1904. Member N. A., 1901; S. A. A., 1852; N. S. S. (pres. 1904-5); N. Y. Arch. Lg., 1890; A. I. A. (cor.), 1896; Academy, San Luca, Rome; N. A. C.; Century Assoc.; Nat. Ins. A. L.

506. Head from Hunt Memorial.

HARVEY, ELI.-Born Ogden, O., 1860. Pupil of Cincinnati Academy under Lentz, Noble and Rebisso. Julian Academy in Paris under Lefebrre, Constant and Doucet; Delecluse Academy under Delance and Callot and of Fremiet at the Jardin des Paintes. First Class Gold Medal for Painting, Paris Province Exposition, 1900; Wannamaker Prize for Sculpture; Paris A. A. A.; Bronze Medal for Sculpture, Pan-American Exposition, 1901. Member N. S. S.; N. Y. Arch. Lg., 1903; Minicipal A. S.

507. Maternal Caress.

508. American Elk.

Loaned by Bureau Bros., Philadelphia.

509. Russian Brown Bear Amusing Her Young.

510. Recumbent Lioness.

511. Contentment-Indian Leopardess with Cubs.

512. Group of Playing Leopards.

513. Lion in the Desert.

514. Drinking Bear. 
LUKEMAN, AUGUSTUS-Born Richmond, Va., 1870. Pupil of D. C. French in New York; Ecole des Beaux Arts in Paris. Bronze Medal, St. Louis Exposition, 1904; Member N. Y. Arch Lg.; N. S. S.; N. A. C.

515. The Finding of Moses.

PAINE, RICHARD G.

516. Teasing-Puma and Turtle.

517. Sentinel Lion.

PROCTOR, A. PHIMISTER-Born Bozanquit, Ontario, Canada, 1862. Pupil of N. A. D. and A. S. L. in New York; Peuch and Injalbert in Paris. Reinhart Scholarship; Medal, Columbian Exposition, Chicago, 1893; Gold Medal, Paris Exposition, 1900; Member Jury of Awards Pan-American Exposition, Buffalo, 1901; Gold Medal for Sculpture and Bronze Medal for Painting, St. Louis Exposition, 1904. Member N. A., 1904 ; S. A. A., 1895; A. W. C. S.; N. Y. Arch. Lg., $1899 ;$ N. S. S.; A. Aid S.; Century Assoc. Specialty, Animals.

518. Mounted Indian.

DEMING, EDWIN WILLARD-(See 151.)

519. Toiler of the Plain.

520. The Fight.

521. The Honey Bear.

522. The Mutual Surprise.

523. The Bear and the Turtle.

VONNOH, BESSIE POTTER-Born St. Louis, Mo., 1872. Pupil of A. I. C. under Taft. Second Prize, Nashville Exposition, 1897; Bronze Medal, Paris Exposition, 1900; Honorable Mention Pan-American Exposition, Buffalo, 1901; Gold Medal, St. Louis Exposition, 1904 ; Julia Shaw Prize, N. A. D., 1904. Member N. S. S.; A. N. A., 1906. Specialty, Small Groups.

524. A Modern Madonna:

525. Cinderella.

526. Mother and Child.

527. A sketch.

528. Girl Reading.

RUSSELL, CHARLES M.-Great Falls, Montana.

529. Scalp Dance.

Loaned by the Roman Bronze Works, Brooklyn, N. Y.

530. Buffalo Hunt.

Loaned by the Roman Bronze Works, Brooklyn, N. Y.

531. Finishing Coup.

Loaned by the Roman Bronze Works, Brooklyn, N. Y. 


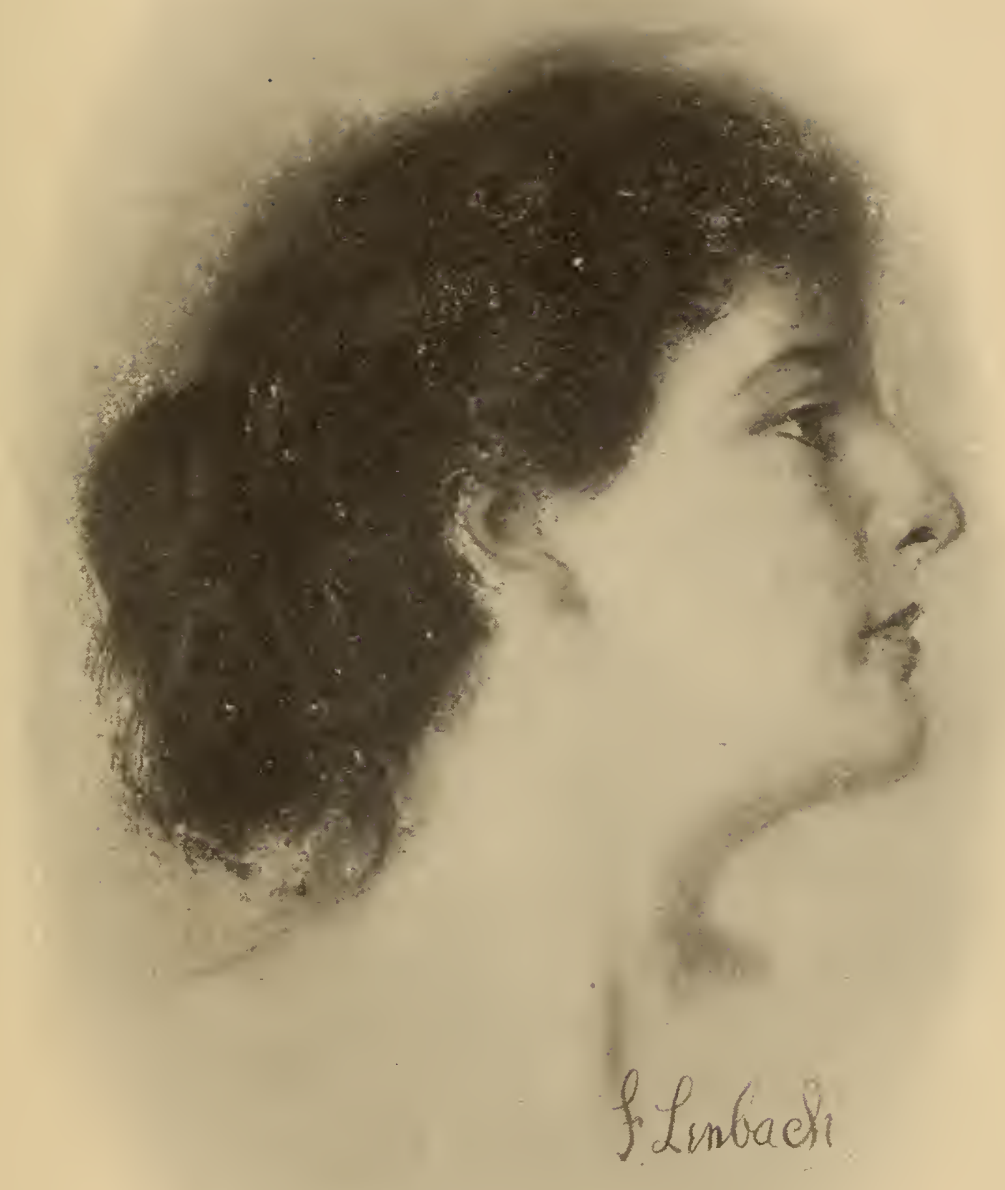

MRS. TREAT

Franz von Lenbach 



\section{ABBREVIATIONS}

A. A. S.-American Art Society, Philadelphia.

A. Aid S.-Artist's Aid Society of New York.

A. C. P.-Art Club of Philadelphia.

A. Fund S.-Artists' Fund Society, New York.

A. I. A.-American Institute of Architects.

A. I. C.-Art Institute of Chicago.

A. N. A.-Associate National Academy of Design, New York.

Arch. L. A.-Architectural League of America.

A. S. L. of N. Y.-Art Students' League of New York.

A. W. C. S.-American Water Color Society, New York.

B. A. C.-Boston Art Club.

Brooklyn A. C.-Brooklyn Art Club.

C. I.-Carnegie Institute, Pittsburg.

Chicago S. A.-Chicago Society of Artists.

Copley S.-Copley Society of Boston.

Corcoran-Corcoran Art Gallery, Washington, D. C.

Fellowship P. A. F. A.-Fellowship Pennsylvania Academy of Fine Arts.

Mural P.-National Society of Mural Painters, New York.

Nat. Inst. A. L.-National Institute of Arts and Letters.

N. A.-National Academy of Design, New York.

N. A. C.-National Arts Club, New York.

N. A. D.-National Academy of Design, used to designate school.

Nat. Gal.-National Gallery, Washington, D. C.

N. S. S.-National Sculpture Society, New York.

N. Y. Arch. Lg.-Architectural League of New York.

N. Y. W. C. C.-New York Water Color Club.

P. A. F. A.-Pennsylvania Academy of Fine Arts, Philadelphia.

Paris A. A. A.-American Art Association of Paris.

Paris S. A. P.-Paris Society of American Painters.

Phila. W. C. C.-Philadelphia Water Color Club.

Plastic C.-Plastic Club of Philadelphia.

S. A. A.- Society of American Artists, New York.

S. I.-Society of Illustrators, New York.

Salma. C.-Salmagundi Club, New York.

S. W. A.- Society of Western Artists.

S. Washington A.- Society of Washington (D. C.) Artists.

Washington W. C. C.-Washington (D. C.) Water Color Club. 



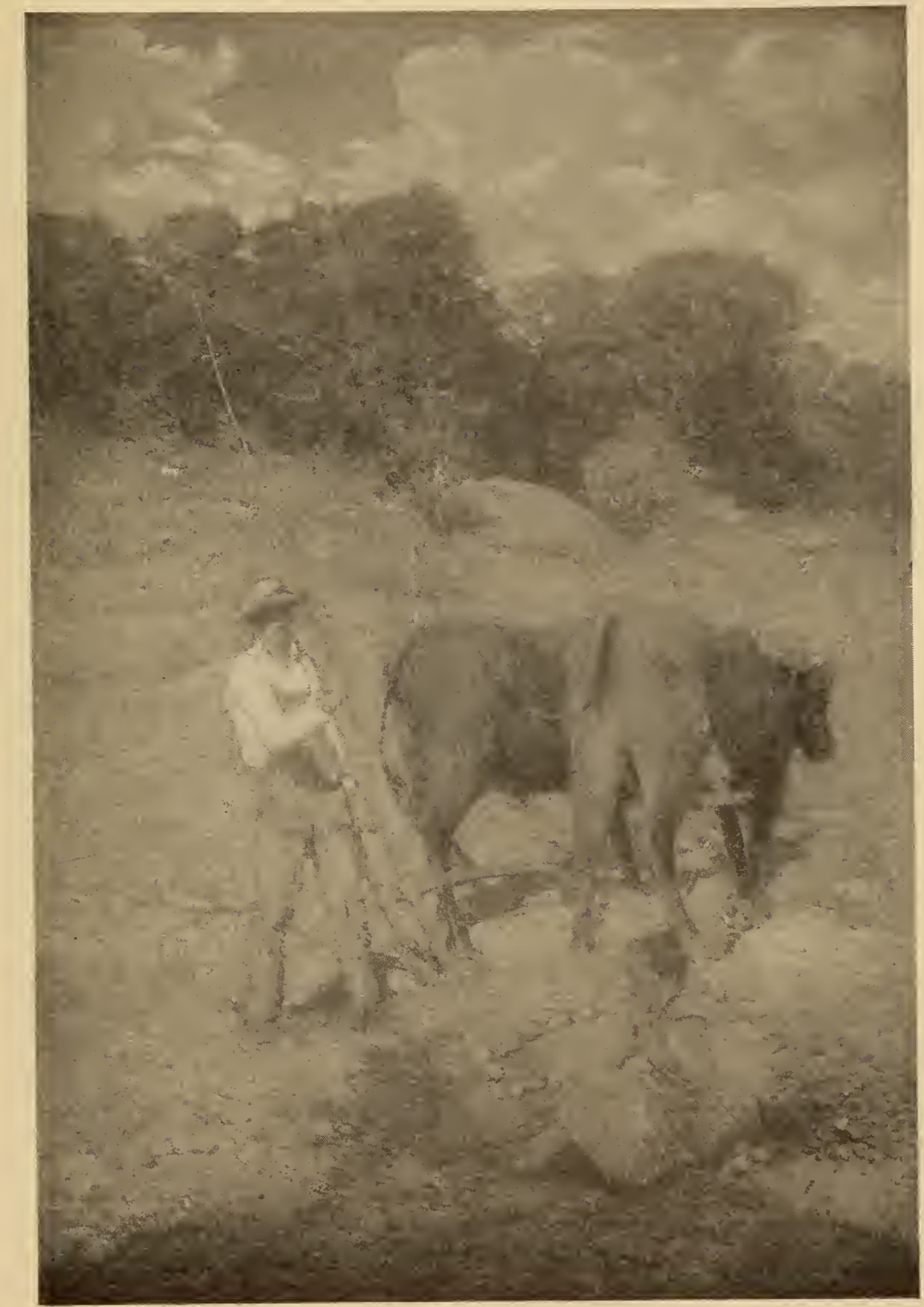

PLOUGHING FOR BUCKWHEAT.

J. Alden Weir. 



\section{INDEX OF ARTISTS}

The letters indicate the Gallery; the figures the number of the picture.

Alexander, John W. E $23 \pi$, G 323

Allori, Cristoforo (called Il Bronzino). A 17

Alma-Tadema, Sir Lawrence C 107

Andre, Albert F 270,278

Anshutz, Thomas P. E 244

Ballin, Hugo F 271,291, G 340

Barillot, Leon

Battoni, Pompeo Girolamo B 87

Beal, Gifford .H $381,388,403,410,417$

Beckwith, J Carroll E 226, F 305

Benson, Frank W.

C 124, D 194

Blakelock, Ralph A. C $118, F 260,293$, G 343

Blommers, Bernardus Johannes

D 174, E 221 , G 358

Bogert, George H. F 268

Bonifazio, Veronese D 163, G 326,328

Bonnat, Leon A 34

Botticelli, Allessandro

L. Atrium 103

Boudin, Eugene A 38

Breckenridge, Hugh $\mathrm{H}$. F 264, 302, H 375

E 232, F 309

Breton, Jules A 15

Brush, George De Forest G 363

Buffet, Amedee H 383

Buffet, Paul H $\quad 387,389$

Bunce, William Gedney G 335

Canaletto, Antonio Canal A 29

Carracei, Ludovico B 73

Carlsen, Emil D 189, G $348,366,367$

Cassatt, Mary E 220

Chabas, Paul Emile H 412

Charpin, Albert H 411

Chretien, Rene Louis H 406

Church, Frederic Edwin F 281

Church, Frederick Stuart

C 155

Clays, Paul Jean

E 208

Coffin, William A.

C 133 
Constable, John

A 22,33

Cooper, Colin Campbell

E 242, F 294

Cooper, Emma Lampert

D 171

Cornoyer, Paul

F 283

Corot, J. B. C.

A $2,4,13,14,20,27,37, \mathrm{~B} 62,96, \mathrm{H} 376,394$

Correggio, Antonio Allegri

A 49

Courbet, Gustave

B $74, \quad$ H 372

Couse, E. Irving

C 121, E 247 , G 351

Couture, Thomas

B 70

Crane, Bruce

E 257, F 295

Crane, Frederick

C $120, \quad$ F 284

Crome, John

B 57

Cuyp, Jacob Gerritse (The EIder)

A. 12

Daubigny, Charles Francois

B $85,89,93$

Davies, Arthur B.

Davis, Charles $\mathrm{H}$

C 130, D 177, F 282

C 142, E 235,249

D 197 , G 352

Dearth, Henry Golden

E 243

De Bock, Theophile

D 172, F 275, G 330
H 296
A 25, B 83, H 402

De Haven, Frank

D 172, F 275, G 330
F 296
A 25, B 83, H 402

De Hoog, Bernard

Delachaux, Leon

D 172, F 275, G 330
F 296
A 25, B 83, H 402

Delacroix, F. V. E.

Deming, Edward Willard

D'Espagnat, Geor'ges

Dessar, Louis Paul

Dewey, Charles Melville

Dewing, Thomas

C $151,519,520,521,522,523$
E 223, U. Atrium 312

C 111 , E 218

De Wint, Peter

D $167,186,187,190,193$

F 265, G 365

B 72

Diaz, Narcisse Virgi!c

Didier-Pouget, WV.

A 3,30, B $60,82,95$, H 373,396

C 145

Dieterle, Marie

E 253

Dobson, WVilliam

A 31

Domingo, J.

C 132

Dougherty, Paul

Du Mond, Frank Vincent

Dupre, Jules

D 168, F 297, G 362

Dupre, Julian

A $10,35, \mathrm{~B} 71,75$

Dustin, Silas S.

C. Atrium 318

Eaton, Charles Warren

Eberle, Abastenia St. L.

C 154

Evans, Rudolph

, G 342

Foster, Ben

505

French, Daniel C.

183

Gagliardini, Julien G.

506

Gainsborough, Thomas

H 378,392

Gale, William

159

Gay, Edward

E 246

Genth, Lillian M.

F 288

Gerome, Jean Leon

E 224

Gosselin, Albert

6397

Greco, El

A 23

Grimlund, Johannes

.H 422

Groll, Albert I,

C 123 , E 259

Gruppe, Charles P.

D) 179, G $319,327,350$ 


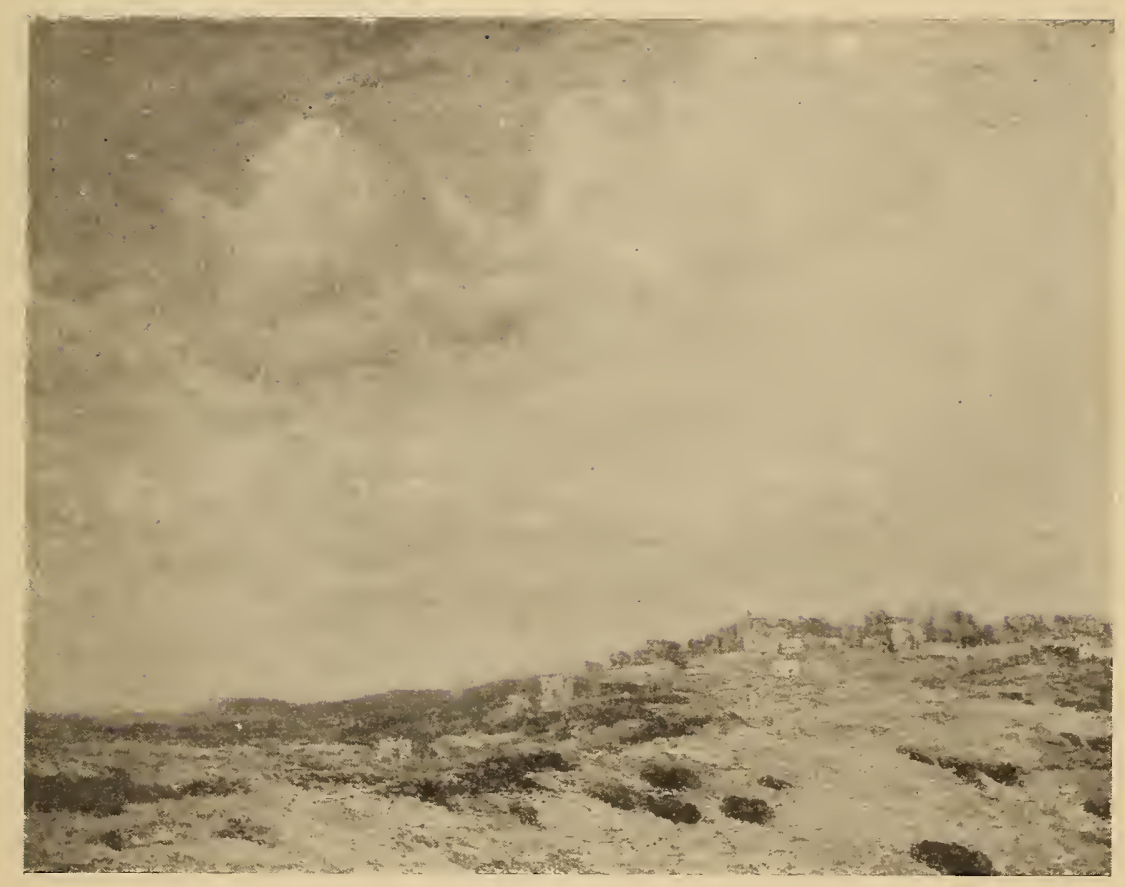



Huguet, Victor Pierre

C 138, F 300

Inness, George

Inness, George, Jr.

D 164,198, G 346

Isabey, Eugene

Jacque, Charles

Kensett, John Fređerick

Martin, Homer D.

Melchers, Gari

Michel, Georges 
Morland, George

A 19, B 77

Murillo, Bartholome Esteban

B 88

Murphy, Herman Dudley

E 219,254

Murphy, J. Francis

D 161,199, G 361

Mytens, Daniel (The Elder)

B 97

Nattier, Jean Marc

B 56

Nettleton, Walter

E 248, U. Atrium 313

Newell, George Glenn

G 339,341

Ochtman, Leonard

Opie, John

Paine, Richard G.

Palmer, Walter L.

516,517

Pasini, Alberto

E 209, 241

Paterson, James

H 418

C 113

Paxton, William M.

E 215

Pelouse, Leon Germain

H $395,407,408,409$

Perrault, Leon

H 414,419

Phillippoteau, Paul

A 51

Picknell, William Lamb

L. Atrium 101

Pissarro, Camille

E 245

Poțter, Paul

B 92

Potthast, Edward H.

Pourbus, Frans (The Younger)

C 126, E 206

B 94

Proctor, Alexander Phimister

518

Ranger, Henry W.

C 146, G 338

Ravanne, Leon G.

H 415

Redfield, Edward W.

Rehn, Frank K. M.

D 169,182, E 228, G 331

Reid, Robert

C 143, D 196

Rembrandt van Rijn

A 43

Reynolds, Sir Joshua

B 61,100

Ribera, Josef de (Lo Spagnoletto)

A 28

Richter, Edouard

B 69

Rico, Martin

E 239

Rieder, $M$.

H 379

Rigolot, Albert G.

H 399

Ritschel, William

E 216,222

H. 382

Robert-Fleury, Tony

Robinson, William S.

Romney, George

Rousseau, Theodore

Rubens, Peter Paul

Russell, Charles M.

Ryder, Chauncey F.

Sargent, John Singer

Sartain, William

$\mathrm{D} 188, \mathrm{E} 256, \mathrm{G} 332$
$\mathrm{~A} 40, \mathrm{~B} 64$

D 188, E 256, G 332

A 16,32, B 55, H 393

B 84

$529,530,531$

Schram, A. S.

Schreyer, Adolphe

Schreyvogel, Charles

Shirlaw, Walter

$213, G 357$

F 279,287

C 135

A $26, \mathrm{~B} 5 \mathrm{~S}$

Sisley, Alfred

U. Atrium 316

Sloan, John

.D 180

Sloane, George

E 234

Smedley, William 'T.

G 345

D 178 
Smillie, George $\mathrm{H}$.

D. 170

Smith, W. Granville

D 185,192, G $320,347,360$

Sousa-Pinto, Jose

.H 391

Stevenson, R. Macauley

.D 200

Tanner, Henry Ossawa

D 166, G 324

Tattegrain, Francis

H 421

Teniers, David (The Younger)

B 68

Ter Meulen, Francois Pieter

C 156

Thaulow, Fritz

C 114

Thomas, Grosvenor

G 321

Tintoretto, Il

B 76

Troyon, Constant

A 18

Tryon, Dwight

C 119

Turner, J. M. W.

A $45, B 90$

Twachtman, John H.

C $106,116, \mathrm{D} 158, \mathrm{~F} 274, \mathrm{G} 334$

Van Dyck, Sir Anthony

A 47,48

Tran Laer, Alexander $\mathrm{T}$.

C 112, D 176

Van Marcke, Emile

A 21

Van Ravensteyn, Jan

A 36

Vernet, Claude Joseph

A 39

Vibert, Jehan Georges

B 63,99

Volk, Douglas

G 337,349

Vonnoh, Bessie Potter

Wagrez, Jacques C.

.H 404

Walker, Horatio

G 364,368

Wallet, Albert Charles

.H 413,420

Watrous, Harry Wilson

E 255

Watts, George F.

B 66

Waugh, Frederick J.

L. Atrium 105, C 134

Weeks, Edwin Lord

D 181

Weir, J. Alden

WVeiss, Jose

C 129, D 202, E 217, F 273, G 370

C 131

Wiegand, Gustave

Wiggins, Carleton

D 195, U. Atrium 314

F 298, 301

Wiles, Irving $\mathbf{R}$.

D $204, G 333$

Williams, Frederick Ballard

Wilson, Richard

B 59

Woodbury, Charles

E 252, U. Atrium 315

Wyant, Alexander $\mathrm{H}$.

E $252, \mathrm{U}$. Atrium 315
G 325,354

Yates, Cullen

Zandomeneghi, Frederico

C 147,152, G 322

Ziem, Felix

E 258, F 306

Zuber, Jean Henri

A $9, B 80$

H 400,405 



\section{Finding List of Those Loaning Pictures}

Albright Art Gallery Buffalo, N. Y. Arnheim, Mark New York City Art Institute of Chicago Chicago, Ill. Ayer, W. B. Portland, Oregon Blakeslee Galleries New York City Bushnell, Charles E. New York City Bureau Bros. Philadlephia, Pa. Carey, Judge C. H. Portland, Oregon Chapman, Henry T. Brooklyn, N. Y. Clise, H. R. Seattle Clise, J. W. Seattle Crane, Frederick. New York Dowden, George A.

Drant, W. H. Newark, N. J. Durand-Ruel \& Sons ............................................................ York City Dustin, Silas S. ........................................................................... York City

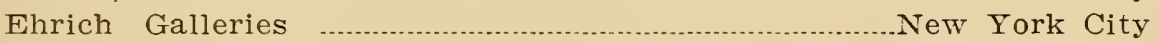
Erans, William T. Montclair, N. J. Folsom Company New York City Frye, C. H. Haggin, J. B. Seattle Hearn, George A. New York City New York City Honeyman, Mrs. David T. Portland, Oregon Hurley, William S. Hutchinson, Charles L.

Brooklyn, N. Y. Kelley, Mrs. P. F. Kerry, A. S. Chicago, Ill. Lathrop, Francis Linde, Mrs. Kate Macbeth, Wiiliam Mansfield, Burton Seattle Seattle New York City Hoboken, N. J. New York City McDermott, Frank Sonttle McGraw, John H. Pacific Trust Co. Seattle Prinz Bros. Seattle Raymond, Dr. Alfred Brooklyn, N. Y. Seattle Roman Bronze Works Sanden, A. T. Goshen, N. Y. 
Sander, Fred E. seattle

Schaus Galleries

Seward, Wm. H.

Shipman, C. M.

Smith, C. J.

Stimson, C. D.

Stimson, F. S.

'Treat, Harry Whitney

Trevor, William

Vose, R. C. \& N. M.

Vose, S. M.

Walker, M. H.

Walker, T. B.

Warren, Mrs. Fiske

White, A. L.

White, C. Fr.

Wood, C. E. S.

IVyant, A. L.

New York City Auburn, N. Y. Newark, N. J. Seattle Seattle Seattle Seattle

New York City Boston, Mass. Providence, R. I. Salt Lake City, Utah Minneapolis, Minn. Boston, Mass. Spokane, Wash. Seattle Portland, Oregon New York City 




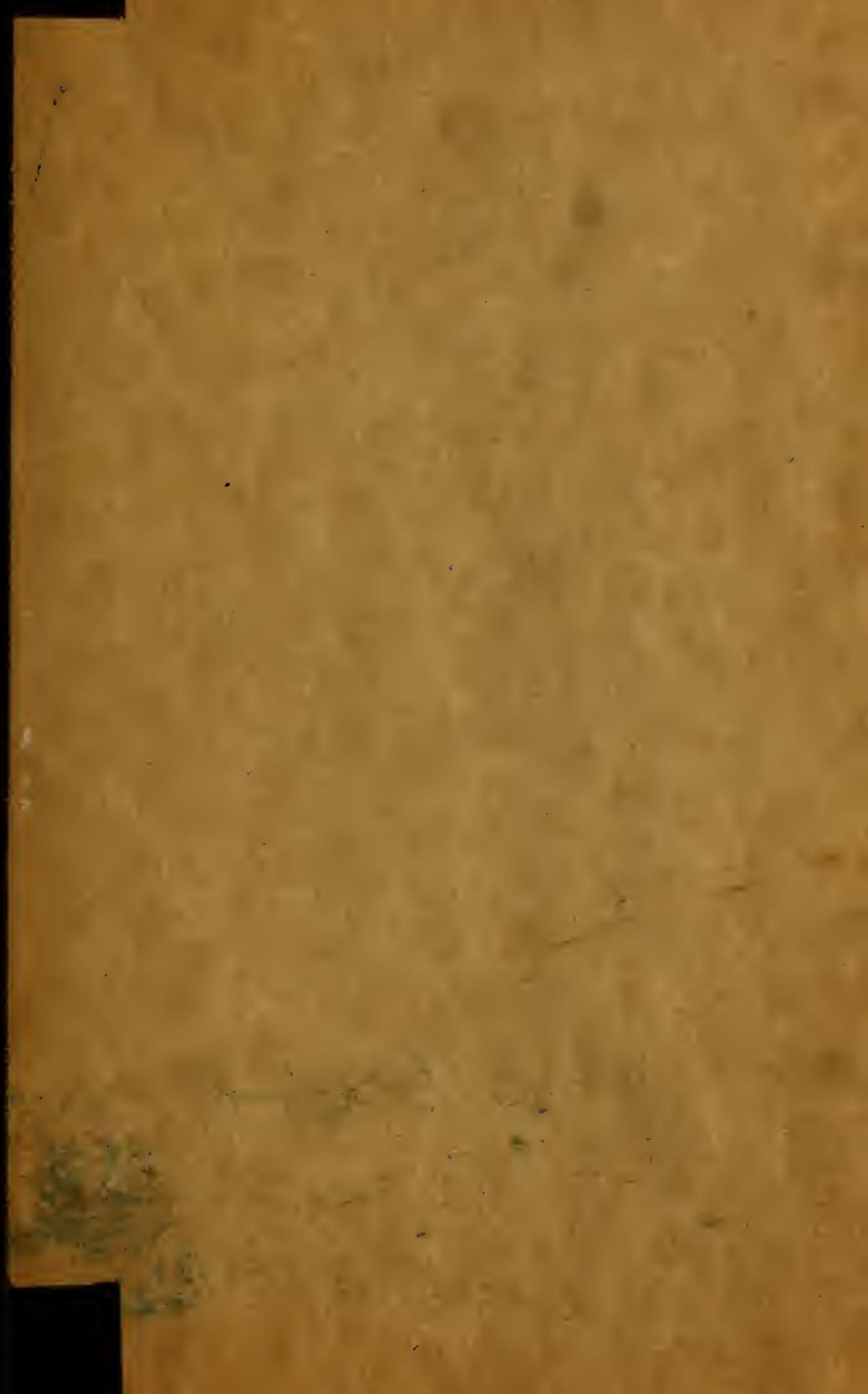


